\title{
Mass formula and Oort's conjecture for supersingular abelian threefolds
}

\author{
Valentijn Karemaker ${ }^{\mathrm{a}}$, Fuetaro Yobuko ${ }^{\mathrm{b}}$, Chia-Fu $\mathrm{Yu}^{\mathrm{c}, *}$ \\ a Mathematical Institute, Utrecht University, Utrecht, the Netherlands \\ b Graduate School of Mathematics, Nagoya University, Nagoya, Japan \\ c Institute of Mathematics, Academia Sinica and National Center for Theoretic \\ Sciences, Taipei, Taiwan
}

\section{A R T I C L E I N F O}

\section{Article history:}

Received 13 August 2020

Received in revised form 7 April

2021

Accepted 17 May 2021

Available online 2 June 2021

Communicated by Zhiwei Yun

\section{Keywords:}

Mass formulae

Supersingular abelian varieties

Automorphism groups

\section{A B S T R A C T}

Katsura and Oort obtained an explicit description of the supersingular locus $\mathscr{S}_{3,1}$ of the Siegel modular variety of degree 3 in terms of class numbers. In this paper we study an alternative stratification of $\mathscr{S}_{3,1}$, the so-called mass stratification. We show that when $p \neq 2$, there are eleven strata (one of $a$-number 3 , two of $a$-number 2 and eight of $a$-number 1 ). We give an explicit mass formula for each stratum and classify possible automorphism groups on each stratum of $a$-number one. On the largest open stratum we show that every automorphism group is $\{ \pm 1\}$ if and only if $p \neq 2$; that is, we prove that Oort's conjecture on the automorphism groups of generic supersingular abelian threefolds holds precisely when $p>2$.

(C) 2021 Elsevier Inc. All rights reserved.

\footnotetext{
* Corresponding author.

E-mail addresses: V.Z.Karemaker@uu.nl (V. Karemaker), yobuko@math.nagoya-u.ac.jp (F. Yobuko), chiafu@math.sinica.edu.tw (C.-F. Yu).
} 


\section{Introduction}

Throughout this paper, let $p$ be a prime number, and let $k$ be an algebraically closed field of characteristic $p$. An abelian variety $X$ over $k$ is said to be supersingular if it is isogenous to a product of supersingular elliptic curves; it is called superspecial if it is isomorphic to a product of supersingular elliptic curves. To each polarised supersingular abelian variety $x=\left(X_{0}, \lambda_{0}\right)$ of $p$-power polarisation degree, we associate a set $\Lambda_{x}$ of isomorphism classes of $p$-power degree polarised abelian varieties $(X, \lambda)$ over $k$, consisting of those whose associated quasi-polarised $p$-divisible groups satisfy $(X, \lambda)\left[p^{\infty}\right] \simeq\left(X_{0}, \lambda_{0}\right)\left[p^{\infty}\right]$. It is known that $\Lambda_{x}$ is a finite set, and the mass of $\Lambda_{x}$ is defined to be the weighted sum

$$
\operatorname{Mass}\left(\Lambda_{x}\right):=\sum_{(X, \lambda) \in \Lambda_{x}} \frac{1}{|\operatorname{Aut}(X, \lambda)|}
$$

Let $\mathscr{A}_{g}$ be the moduli space over $\overline{\mathbb{F}}_{p}$ of $g$-dimensional principally polarised abelian varieties. If $x=\left(X_{0}, \lambda_{0}\right)$ is a superspecial point in $\mathscr{A}_{g}(k)$, that is, $X_{0}$ is superspecial, then $\Lambda_{x}$ coincides with the superspecial locus $\Lambda_{g, 1}$ of $\mathscr{A}_{g}$, which consists of all superspecial points in $\mathscr{A}_{g}$, called the principal genus. The classical mass formula (see HashimotoIbukiyama [6, Proposition 9] and Ekedahl [3, p. 159]) states that

$$
\operatorname{Mass}\left(\Lambda_{g, 1}\right)=\frac{(-1)^{g(g+1) / 2}}{2^{g}}\left\{\prod_{i=1}^{g} \zeta(1-2 i)\right\} \cdot \prod_{i=1}^{g}\left\{\left(p^{i}+(-1)^{i}\right\}\right.
$$

where $\zeta(s)$ denotes the Riemann zeta function.

More generally, for any integer $c$ with $0 \leq c \leq\lfloor g / 2\rfloor$, let $\Lambda_{g, p^{c}}$ denote the finite set of isomorphism classes of $g$-dimensional polarised superspecial abelian varieties $(X, \lambda)$ such that $\operatorname{ker}(\lambda) \simeq \alpha_{p}^{2 c}$, where $\alpha_{p}$ is the kernel of the Frobenius morphism on the additive group $\mathbb{G}_{a}$. Then one also has $\Lambda_{g, p^{c}}=\Lambda_{x}$ for any member $x$ in $\Lambda_{g, p^{c}}$. The case $c=\lfloor g / 2\rfloor$ is called the non-principal genus. As shown by Li-Oort [13], both the principal and nonprincipal genera describe the irreducible components of the supersingular locus $\mathscr{S}_{g, 1}$ of $\mathscr{A}_{g}$. Similarly, the sets $\Lambda_{g, p^{c}}$ describe the irreducible components of supersingular Ekedahl-Oort (EO) strata in $\mathscr{A}_{g}$ cf. [4]. The explicit determination of the class number $\left|\Lambda_{g, p^{c}}\right|$, i.e., the class number problem, is a very difficult task for large $g$, and is still open for $g=3$ and $c=1$. Nevertheless, an explicit calculation of the mass $\operatorname{Mass}\left(\Lambda_{g, p^{c}}\right)$ is more accessible and provides a good estimate for the class number. This mass was calculated explicitly by the third author [23, Theorem 1.4] when $g=2 c$ and extended to arbitrary $g$ and $c$ by Harashita [4, Proposition 3.5.2].

In [27], J.-D. Yu and the third author explicitly calculated the mass formula for $\operatorname{Mass}\left(\Lambda_{x}\right)$ for an arbitrary principally polarised supersingular abelian surface $x=$ $\left(X_{0}, \lambda_{0}\right)$. In [8], Ibukiyama investigated principal polarisations of a given supersingular 
non-superspecial abelian surface $X_{0}$. He explicitly computed the number of polarisations and the mass of the corresponding principally polarised abelian surfaces. He also showed the agreement with $\left|\Lambda_{x}\right|$ and $\operatorname{Mass}\left(\Lambda_{x}\right)$ cf. [8, Proposition 3.3 and Theorem 3.6], respectively, for a member $x=\left(X_{0}, \lambda_{0}\right)$ in $\mathscr{S}_{2,1}$. As an important arithmetic application, Ibukiyama proved Oort's conjecture that the automorphism group of any generic member is $\{ \pm 1\}$ for $p \geq 3$, and he gave a counterexample for $p=2$.

Inspired by Ibukiyama's work [8], and as a continuation of [27], in this paper we completely determine the mass formula for $\operatorname{Mass}\left(\Lambda_{x}\right)$ when $g=3$, and prove Oort's conjecture for $p>2$ as an arithmetic application. To describe our results, we introduce some notation; more details will be given in Sections 2 and 3 .

For any abelian variety $X$ over $k$, the a-number of $X$ is $a(X):=\operatorname{dim}_{k} \operatorname{Hom}\left(\alpha_{p}, X\right)$. For abelian threefolds $X$ we have $a(X) \in\{1,2,3\}$; when computing the mass, we will separate into cases based on the $a$-number.

Further let $E$ be a supersingular elliptic curve over $\mathbb{F}_{p^{2}}$ with Frobenius endomorphism $\pi_{E}=-p$, and let $E_{k}=E \otimes_{\mathbb{F}_{p^{2}}} k$. For each integer $c$ with $0 \leq c \leq\lfloor g / 2\rfloor$, we denote by $P_{p^{c}}\left(E_{k}^{g}\right)$ the set of polarisations $\mu$ on $E_{k}^{g}$ such that $\operatorname{ker} \mu \simeq \alpha_{p}^{2 c}$; one has $P_{p^{c}}\left(E_{k}^{g}\right)=$ $P_{p^{c}}\left(E^{g}\right)$. As superspecial abelian threefolds are unique up to isomorphism, there is a natural bijection $P_{p^{c}}\left(E_{k}^{g}\right) \simeq \Lambda_{g, p^{c}}$.

Let $\mu$ be a polarisation in $P_{1}\left(E_{k}^{3}\right)$. As alluded to above, Li and Oort [13] show there is a one-to-one natural correspondence between the set $P_{1}\left(E_{k}^{3}\right)$ and the set $\Sigma\left(\mathscr{S}_{3,1}\right)$ of (geometrically) irreducible components of $\mathscr{S}_{3,1}$. More precisely, they consider the moduli space $\mathscr{P}_{\mu}$ (resp. $\mathscr{P}_{\mu}^{\prime}$ ) over $\mathbb{F}_{p^{2}}$ of three-dimensional (resp. rigid) polarised flag type quotients with respect to $\mu$. This space is an irreducible scheme which comes with a proper projection morphism $\mathrm{pr}_{0}: \mathscr{P}_{\mu} \rightarrow \mathscr{S}_{3,1}$, such that for each principally polarised supersingular abelian threefold $(X, \lambda)$ there exist a $\mu \in P_{1}\left(E_{k}^{3}\right)$ and a $y \in \mathscr{P}_{\mu}$ such that $\operatorname{pr}_{0}(y)=[(X, \lambda)] \in \mathscr{S}_{3,1}$.

Let $C \subseteq \mathbb{P}^{2}$ be the Fermat curve of degree $p+1$ defined by the equation $X_{1}^{p+1}+$ $X_{2}^{p+1}+X_{3}^{p+1}=0$. There exists a natural proper morphism $\pi: \mathscr{P}_{\mu} \rightarrow C$ with $\mathbb{P}^{1}$-fibres, and it is shown (cf. [13, Section 9.4] and Proposition 3.7) that $\mathscr{P}_{\mu}$ is isomorphic to the $\mathbb{P}^{1}$-bundle $\mathbb{P}_{C}(\mathscr{O}(-1) \oplus \mathscr{O}(1))$ over the Fermat curve $C$. Moreover, the morphism $\pi$ has a section $s: C \stackrel{\sim}{\longrightarrow} T \subseteq \mathscr{P}_{\mu}$, cf. Definition 3.14. In particular, for each $k$-point $(X, \lambda)$ in the component $\operatorname{pr}_{0}\left(\mathscr{P}_{\mu}\right)$ of $\mathscr{S}_{3,1}$ and a point $y \in \mathscr{P}_{\mu}(k)$ lying over $(X, \lambda)$, there exists a unique pair $(t, u)$ where $t=\left(t_{1}: t_{2}: t_{3}\right) \in C(k)$ and $u=\left(u_{1}: u_{2}\right) \in \pi^{-1}(t) \simeq \mathbb{P}_{t}^{1}(k)$ that characterises $y$. Moreover, we have (cf. Proposition 3.15):

(1) If $y \in T$ then $a(X)=3$.

(2) For any $t \in C(k)$, we have $t \in C\left(\mathbb{F}_{p^{2}}\right)$ if and only if for any $y \in \pi^{-1}(t)$ the corresponding threefold $X$ has $a(X) \geq 2$.

(3) We have $a(X)=1$ if and only if $y \notin T$ and $\pi(y) \notin C\left(\mathbb{F}_{p^{2}}\right)$.

We are now ready to state our first two main results, computing the mass for any principally polarised supersingular abelian threefold. 
Theorem A. (Theorem 4.3) Let $x=(X, \lambda) \in \mathscr{S}_{3,1}(k)$ with $a(X) \geq 2$, let $\mu \in P_{1}\left(E^{3}\right)$, and let $y \in \mathscr{P}_{\mu}^{\prime}(k)$ be such that $\operatorname{pr}_{0}(y)=[(X, \lambda)]$. Write $y=(t, u)$ where $t=\pi(y) \in C\left(\mathbb{F}_{p^{2}}\right)$ and $u \in \pi^{-1}(t) \simeq \mathbb{P}_{t}^{1}(k)$. Then

$$
\operatorname{Mass}\left(\Lambda_{x}\right)=\frac{L_{p}}{2^{10} \cdot 3^{4} \cdot 5 \cdot 7}
$$

where

$$
L_{p}= \begin{cases}(p-1)\left(p^{2}+1\right)\left(p^{3}-1\right) & \text { if } u \in \mathbb{P}_{t}^{1}\left(\mathbb{F}_{p^{2}}\right) \\ (p-1)\left(p^{3}+1\right)\left(p^{3}-1\right)\left(p^{4}-p^{2}\right) & \text { if } u \in \mathbb{P}_{t}^{1}\left(\mathbb{F}_{p^{4}}\right) \backslash \mathbb{P}_{t}^{1}\left(\mathbb{F}_{p^{2}}\right) \\ 2^{-e(p)}(p-1)\left(p^{3}+1\right)\left(p^{3}-1\right) p^{2}\left(p^{4}-1\right) & \text { if } u \notin \mathbb{P}_{t}^{1}\left(\mathbb{F}_{p^{4}}\right)\end{cases}
$$

where $e(p)=0$ if $p=2$ and $e(p)=1$ if $p>2$.

Theorem B. (Theorem 5.21) Let $x=(X, \lambda) \in \mathscr{S}_{3,1}(k)$ such that $a(X)=1$ and $x \in \operatorname{pr}_{0}\left(\mathscr{P}_{\mu}\right)$ for some $\mu \in P_{1}\left(E^{3}\right)$. Consider an element $y \in \mathscr{P}_{\mu}(k)$ over $x$, which is characterised by the pair $(t, u)$ with $t \in C(k) \backslash C\left(\mathbb{F}_{p^{2}}\right)$ and $u \in \mathbb{P}_{t}^{1}(k)$. Let $\mathscr{D}_{t}$ be as in Definition 5.16, and let $d(t)$ be as in Definition 5.12. Then

$$
\operatorname{Mass}\left(\Lambda_{x}\right)=\frac{p^{3} L_{p}}{2^{10} \cdot 3^{4} \cdot 5 \cdot 7}
$$

where

$$
L_{p}= \begin{cases}2^{-e(p)} p^{2 d(t)}\left(p^{2}-1\right)\left(p^{4}-1\right)\left(p^{6}-1\right) & \text { if } u \notin \mathscr{D}_{t} ; \\ p^{2 d(t)}(p-1)\left(p^{4}-1\right)\left(p^{6}-1\right) & \text { if } t \notin C\left(\mathbb{F}_{p^{6}}\right) \text { and } u \in \mathscr{D}_{t} \\ p^{6}\left(p^{2}-1\right)\left(p^{3}-1\right)\left(p^{4}-1\right) & \text { if } t \in C\left(\mathbb{F}_{p^{6}}\right) \text { and } u \in \mathscr{D}_{t} .\end{cases}
$$

The mass function on $\mathscr{S}_{3,1}$ induces a stratification such that the mass function becomes constant on each stratum. By Theorem A, the locus of $\mathscr{S}_{3,1}$ with $a$-number $\geq 2$ decomposes into three strata: one stratum with $a$-number 3 and two strata with $a$-number 2. On the locus with $a$-number 1 , the stratification depends on $p$. When $p \neq 2$, the $d$ invariant takes values in $\{3,4,5,6\}$ and $d(t)=3$ if and only if $t \in C\left(\mathbb{F}_{p^{6}}\right)$. In this case, Theorem B says that the mass function depends only on the $d$-invariant and whether $u \in \mathscr{D}_{t}$ or not, and hence there are eight strata. When $p=2$, the $d$-value $d(t)$ is always 3 and Theorem B gives three strata.

Our computations of the automorphism groups can be summarised as follows.

Theorem C. Let $x=(X, \lambda) \in \mathscr{S}_{3,1}(k)$ and $\mu \in P_{1}\left(E^{3}\right)$ so that $x \in \operatorname{pr}_{0}\left(\mathscr{P}_{\mu}\right)$. Consider an element $y \in \mathscr{P}_{\mu}$ over $x$, which is characterised by the pair $(t, u)$ with $t \in C(k)$ and $u \in \mathbb{P}_{t}^{1}(k)$. Let $\mathscr{D}_{t}$ be as in Definition 5.16 and let $d(t)$ be as in Definition 5.12. 
(1) (Theorem 6.4) Suppose that $a(X)=1$, so that $t \in C(k) \backslash C\left(\mathbb{F}_{p^{2}}\right)$. Assume that $(t, u) \notin \mathscr{D}$, that is, $u \notin \mathscr{D}_{t}$.

(a) If $p=2$, then $\operatorname{Aut}(X, \lambda) \simeq C_{2}^{3}$.

(b) If $p \geq 5$, or $p=3$ and $d(t)=6$, then $\operatorname{Aut}(X, \lambda) \simeq C_{2}$,

where $C_{n}$ denotes the cyclic group of order $n$.

(2) (Theorem 6.9) Suppose that $a(X)=1$ and that $(t, u) \in \mathscr{D}$ with $t \notin C\left(\mathbb{F}_{p^{6}}\right)$.

(a) If $p=2$, then $\operatorname{Aut}(X, \lambda) \simeq C_{2}^{3} \times C_{3}$.

(b) If $p=3$ and $d(t)=6$, then $\operatorname{Aut}(X, \lambda) \in\left\{C_{2}, C_{4}\right\}$.

(c) For $p \geq 5$, we have the following cases:

(i) If $p \equiv-1(\bmod 4)$, then $\operatorname{Aut}(X, \lambda) \in\left\{C_{2}, C_{4}\right\}$.

(ii) If $p \equiv-1(\bmod 3)$, then $\operatorname{Aut}(X, \lambda) \in\left\{C_{2}, C_{6}\right\}$.

(iii) If $p \equiv 1(\bmod 12)$, then $\operatorname{Aut}(X, \lambda) \simeq C_{2}$.

(3) (Proposition 6.12) Let $\Lambda_{3,1}\left(C_{2}\right):=\left\{(X, \lambda) \in \Lambda_{3,1}: \operatorname{Aut}(X, \lambda) \simeq C_{2}\right\}$ be the set of superspecial principally polarised abelian threefolds satisfying Oort's conjecture. Then

$$
\frac{\left|\Lambda_{3,1}\left(C_{2}\right)\right|}{\left|\Lambda_{3,1}\right|} \rightarrow 1 \quad \text { as } p \rightarrow \infty
$$

In particular, Part (1) of Theorem C shows that Oort's conjecture is true precisely for $p \neq 2$. That is, every generic principally polarised supersingular abelian threefold over $k$ of characteristic $\neq 2$ has automorphism group $C_{2}$.

Schemes in this paper are assumed to be locally Noetherian unless stated otherwise.

The organisation of the paper is as follows. Sections 2 and 3 contain preliminaries, respectively on mass formulae and the structure of the supersingular locus $\mathscr{S}_{3,1}$. In particular, the strategy we will follow in later sections to obtain mass formulae is outlined at the end of Section 2. Sections 4 and 5 determine the mass formulae for supersingular abelian threefolds $X$, respectively with $a(X)=2$ (cf. Theorem A) and $a(X)=1$ (cf. Theorem B). The automorphism groups, as well as the implications for Oort's conjecture, are studied in Section 6 (cf. Theorem C). The Appendix contains results of independent interest, concerning a set-theoretic intersection arising in Section 5.

\section{Mass formulae for supersingular abelian varieties}

\subsection{Set-up and notation}

Throughout the paper, let $p$ be a prime number, let $g$ be a positive integer, and let $k$ be an algebraically closed field of characteristic $p$. The ground field for objects studied is $k$, unless stated otherwise.

For a finite set $S$, write $|S|$ for the cardinality of $S$. Let $\alpha_{p}$ be the unique $\alpha$-group of order $p$ over $\mathbb{F}_{p}$; it is defined to be the kernel of the Frobenius morphism on the additive group $\mathbb{G}_{a}$ over $\mathbb{F}_{p}$. For a matrix $A=\left(a_{i j}\right) \in \operatorname{Mat}_{m \times n}(k)$ and integer $r$, write 
$A^{\left(p^{r}\right)}:=\left(a_{i j}^{p^{r}}\right)$ for the image of $A$ under the $r$ th Frobenius map. Denote by $\widehat{\mathbb{Z}}=\prod_{\ell} \mathbb{Z}_{\ell}$ the profinite completion of $\mathbb{Z}$ and by $\mathbb{A}_{f}=\widehat{\mathbb{Z}} \otimes_{\mathbb{Z}} \mathbb{Q}$ the finite adele ring of $\mathbb{Q}$.

Definition 2.1. For any integer $d \geq 1$, let $\mathscr{A}_{g, d}$ denote the (coarse) moduli space over $\overline{\mathbb{F}}_{p}$ of $g$-dimensional polarised abelian varieties $(X, \lambda)$ with polarisation degree $\operatorname{deg} \lambda=d^{2}$. For any $m \geq 1$, let $\mathscr{S}_{g, p^{m}}$ be the supersingular locus of $\mathscr{A}_{g, p^{m}}$, which consists of all polarised supersingular abelian varieties in $\mathscr{A}_{g, p^{m}}$. Then $\mathscr{S}_{g, 1}$ is the moduli space of $g$-dimensional principally polarised supersingular abelian varieties. Denote $\mathscr{S}_{g, p^{*}}=\cup_{m \geq 1} \mathscr{S}_{g, p^{m}}$.

Definition 2.2. (1) If $S$ is a finite set of objects with finite automorphism groups in a specified category, then we define the mass of $S$ to be the weighted sum

$$
\operatorname{Mass}(S):=\sum_{s \in S} \frac{1}{|\operatorname{Aut}(s)|}
$$

(2) For any $x=\left(X_{0}, \lambda_{0}\right) \in \mathscr{S}_{g, p^{*}}(k)$, we define

$$
\Lambda_{x}=\left\{(X, \lambda) \in \mathscr{S}_{g, p^{*}}(k):(X, \lambda)\left[p^{\infty}\right] \simeq\left(X_{0}, \lambda_{0}\right)\left[p^{\infty}\right]\right\}
$$

where $(X, \lambda)\left[p^{\infty}\right]$ denotes the polarised $p$-divisible group associated to $(X, \lambda)$. Then $\Lambda_{x}$ is a finite set; see [22, Theorem 2.1]. The mass of $\Lambda_{x}$ is defined as

$$
\operatorname{Mass}\left(\Lambda_{x}\right)=\sum_{(X, \lambda) \in \Lambda_{x}} \frac{1}{|\operatorname{Aut}(X, \lambda)|}
$$

\subsection{Superspecial mass formulae}

Recall that a superspecial abelian variety over $k$ is an abelian variety isomorphic to a product of supersingular elliptic curves.

Definition 2.3. Let $0 \leq c \leq\lfloor g / 2\rfloor$ be an integer. We define $\Lambda_{g, p^{c}}$ to be the set of isomorphism classes of $g$-dimensional superspecial polarised abelian varieties $(X, \lambda)$ whose polarisation $\lambda$ satisfies $\operatorname{ker}(\lambda) \simeq \alpha_{p}^{2 c}$. Its mass is

$$
\operatorname{Mass}\left(\Lambda_{g, p^{c}}\right)=\sum_{(X, \lambda) \in \Lambda_{g, p^{c}}} \frac{1}{|\operatorname{Aut}(X, \lambda)|}
$$

In particular, Mass $\left(\Lambda_{g, p^{c}}\right)$ is a special case of $\operatorname{Mass}\left(\Lambda_{x}\right)$, cf. Definition 2.2. Note that the $p$-divisible group of a superspecial abelian variety of given dimension is unique up to isomorphism. Furthermore, the polarised $p$-divisible group associated to any member in $\Lambda_{g, p^{c}}$ is unique up to isomorphism, cf. [13, Proposition 6.1]. Thus, if $x=(X, \lambda)$ is any member in $\Lambda_{g, p^{c}}$, then we have $\Lambda_{x}=\Lambda_{g, p^{c}}$. 


\section{Theorem 2.4.}

(1) For any $g \geq 1$, we have

$$
\operatorname{Mass}\left(\Lambda_{g, 1}\right)=\frac{(-1)^{g(g+1) / 2}}{2^{g}} \prod_{i=1}^{g} \zeta(1-2 i) \cdot \prod_{i=1}^{g}\left(p^{i}+(-1)^{i}\right)
$$

(2) For any $g \geq 1$ and $0 \leq c \leq\lfloor g / 2\rfloor$, we have

$$
\begin{aligned}
\operatorname{Mass}\left(\Lambda_{g, p^{c}}\right)= & \frac{(-1)^{g(g+1) / 2}}{2^{g}} \prod_{i=1}^{g} \zeta(1-2 i) \cdot \prod_{i=1}^{g-2 c}\left(p^{i}+(-1)^{i}\right) \cdot \prod_{i=1}^{c}\left(p^{4 i-2}-1\right) \\
& \cdot \frac{\prod_{i=1}^{g}\left(p^{2 i}-1\right)}{\prod_{i=1}^{2 c}\left(p^{2 i}-1\right) \prod_{i=1}^{g-2 c}\left(p^{2 i}-1\right)}
\end{aligned}
$$

Proof. (1) See [3, p. 159] and [6, Proposition 9]. (2) This follows from [4, Proposition 3.5.2] by the functional equation for $\zeta(s)$. See also [23] for a geometric proof in the case where $g=2 c$.

Using the fact that $\zeta(-1)=-1 / 12, \zeta(-3)=1 / 120$ and $\zeta(-5)=-1 /(42 \cdot 6)$, we obtain the following corollary.

Corollary 2.5. Let $g=3$.

(1) If $c=0$, then $\Lambda_{g, p^{c}}=\Lambda_{3,1}$ consists of all principally polarised superspecial abelian threefolds, and

$$
\operatorname{Mass}\left(\Lambda_{3,1}\right)=\frac{(p-1)\left(p^{2}+1\right)\left(p^{3}-1\right)}{2^{10} \cdot 3^{4} \cdot 5 \cdot 7}
$$

(2) If $c=1$, then $\Lambda_{g, p^{c}}=\Lambda_{3, p}$ consists of all polarised superspecial abelian threefolds whose polarisation $\lambda$ has $\operatorname{ker}(\lambda) \simeq \alpha_{p} \times \alpha_{p}$, and

$$
\operatorname{Mass}\left(\Lambda_{3, p}\right)=\frac{(p-1)\left(p^{3}+1\right)\left(p^{3}-1\right)}{2^{10} \cdot 3^{4} \cdot 5 \cdot 7}
$$

\subsection{From superspecial to supersingular mass formulae}

For a (not necessary principally) polarised supersingular abelian variety $x=\left(X_{0}, \lambda_{0}\right)$ over $k$, let $G_{x}$ be the automorphism group scheme over $\mathbb{Z}$ associated to $x$; for any commutative ring $R$, the group of its $R$-valued points is defined by

$$
G_{x}(R)=\left\{g \in\left(\operatorname{End}\left(X_{0}\right) \otimes_{\mathbb{Z}} R\right)^{\times}: g^{T} \lambda_{0} g=\lambda_{0}\right\}
$$


Definition 2.6. For a connected reductive group $G$ over $\mathbb{Q}$ with finite arithmetic subgroups and an open compact subgroup $U \subseteq G\left(\mathbb{A}_{f}\right)$, we define its (arithmetic) mass $\operatorname{Mass}(G, U)$ by

$$
\operatorname{Mass}(G, U)=\sum_{i=1}^{h} \frac{1}{\left|\Gamma_{i}\right|}, \quad \Gamma_{i}:=G(\mathbb{Q}) \cap c_{i} U c_{i}^{-1},
$$

where $\left\{c_{1}, \cdots, c_{h}\right\}$ is a set of representatives for the double coset space $G(\mathbb{Q}) \backslash G\left(\mathbb{A}_{f}\right) / U$.

Proposition 2.7. For any object $x=\left(X_{0}, \lambda_{0}\right) \in \mathscr{S}_{g, p^{*}}(k)$, there is a natural bijection of pointed sets

$$
\Lambda_{x} \simeq G_{x}(\mathbb{Q}) \backslash G_{x}\left(\mathbb{A}_{f}\right) / G_{x}(\widehat{\mathbb{Z}})
$$

Moreover, if $(X, \lambda)$ is a member of $\Lambda_{x}$ which corresponds to the class $[c]$ under the bijection, then $\operatorname{Aut}(X, \lambda) \simeq G_{x}(\mathbb{Q}) \cap c G_{x}(\widehat{\mathbb{Z}}) c^{-1}$. In particular, we have

$$
\operatorname{Mass}\left(\Lambda_{x}\right)=\operatorname{Mass}\left(G_{x}, G_{x}(\widehat{\mathbb{Z}})\right),
$$

cf. Definition 2.2.

Proof. See [25, Theorems 2.2 and 4.6]. Also see [27, Proposition 2.1] for a proof sketch.

Definition 2.8. Let $U_{1}, U_{2}$ be two open compact subgroups of $G_{x}\left(\mathbb{A}_{f}\right)$. Then we define

$$
\mu\left(U_{1} / U_{2}\right)=\frac{\left[U_{1}: U_{1} \cap U_{2}\right]}{\left[U_{2}: U_{1} \cap U_{2}\right]} .
$$

Interpreting the mass from Definition 2.6 as the volume of a fundamental domain, with notation as above, we have the following lemma.

Lemma 2.9. Let $U_{1}, U_{2}$ be two open compact subgroups of $G_{x}\left(\mathbb{A}_{f}\right)$. Then their (arithmetic) masses compare as

$$
\operatorname{Mass}\left(G_{x}, U_{2}\right)=\mu\left(U_{1} / U_{2}\right) \operatorname{Mass}\left(G_{x}, U_{1}\right)
$$

Lemma 2.10. Let $X$ be a supersingular abelian variety over $k$. Then there exists a pair $(Y, \varphi)$, where $Y$ is a superspecial abelian variety and $\varphi: Y \rightarrow X$ is an isogeny such that for any pair $\left(Y^{\prime}, \varphi^{\prime}\right)$ as above there exists a unique isogeny $\rho: Y^{\prime} \rightarrow Y$ such that $\varphi^{\prime}=\varphi \circ \rho$.

Dually, there exists a pair $(Z, \gamma)$, where $Z$ is a superspecial abelian variety and $\gamma$ : $X \rightarrow Z$ such that for any pair $\left(Z^{\prime}, \gamma^{\prime}\right)$ as above there exists a unique isogeny $\rho: Z \rightarrow Z^{\prime}$ such that $\gamma^{\prime}=\rho \circ \gamma$. 
Proof. See [13, Lemma 1.8]; also see [24, Corollary 4.3] for an independent proof. The proof of [13, Lemma 1.8] contains a gap; see Remark 3.17 for a counterexample to the argument.

Definition 2.11. Let $X$ be a supersingular abelian variety over $k$. We call the pair $(Y, \varphi$ : $Y \rightarrow X)$ or the pair $(Z, \gamma: X \rightarrow Z)$ as in Lemma 2.10 the minimal isogeny of $X$.

Proposition 2.12. Let $x=(X, \lambda) \in \mathscr{S}_{g, p^{*}}(k)$ and let $\varphi: \widetilde{X} \rightarrow X$ be the minimal isogeny of $X$. Put $\widetilde{x}=(\widetilde{X}, \widetilde{\lambda})$, where $\widetilde{\lambda}:=\varphi^{*} \lambda$. Let $(M,\langle\rangle),,(\widetilde{M},\langle\rangle$,$) denote the quasi-polarised$ (contravariant) Dieudonné module of $X, \widetilde{X}$, respectively. Then $\varphi$ induces an injective map $\varphi^{*}: \operatorname{End}\left(X\left[p^{\infty}\right]\right) \hookrightarrow \operatorname{End}\left(\widetilde{X}\left[p^{\infty}\right]\right)$, or equivalently $\varphi^{*}: \operatorname{End}(M) \hookrightarrow \operatorname{End}(\widetilde{M})$, and we have

$$
\begin{aligned}
\operatorname{Mass}\left(\Lambda_{x}\right) & =\left[\operatorname{Aut}\left((\widetilde{X}, \widetilde{\lambda})\left[p^{\infty}\right]\right): \operatorname{Aut}\left((X, \lambda)\left[p^{\infty}\right]\right)\right] \cdot \operatorname{Mass}\left(\Lambda_{\widetilde{x}}\right) \\
& =[\operatorname{Aut}(\widetilde{M},\langle,\rangle): \operatorname{Aut}(M,\langle,\rangle)] \cdot \operatorname{Mass}\left(\Lambda_{\widetilde{x}}\right) .
\end{aligned}
$$

Here the injective map $\varphi^{*}$ yields the inclusion map $\operatorname{Aut}(M,\langle\rangle,) \subseteq \operatorname{Aut}(\widetilde{M},\langle\rangle$,$) .$

Proof. This may be regarded as a refinement of [22, Theorem 2.7]. Through the isogeny $\varphi$, we may view $G_{\widetilde{x}}(\widehat{\mathbb{Z}})$ and $\varphi^{*} G_{x}(\widehat{\mathbb{Z}})$ as open compact subgroups of the same group $G_{\widetilde{x}}\left(\mathbb{A}_{f}\right)$. Using Proposition 2.7 and Lemma 2.9, we see that

$$
\begin{aligned}
\operatorname{Mass}\left(\Lambda_{x}\right) & =\mu\left(G_{\widetilde{x}}(\widehat{\mathbb{Z}}) / \varphi^{*} G_{x}(\widehat{\mathbb{Z}})\right) \operatorname{Mass}\left(\Lambda_{\widetilde{x}}\right) \\
& =\frac{\left[G_{\widetilde{x}}(\widehat{\mathbb{Z}}): G_{\widetilde{x}}(\widehat{\mathbb{Z}}) \cap \varphi^{*} G_{x}(\widehat{\mathbb{Z}})\right]}{\left[\varphi^{*} G_{x}(\widehat{\mathbb{Z}}): G_{\widetilde{x}}(\widehat{\mathbb{Z}}) \cap \varphi^{*} G_{x}(\widehat{\mathbb{Z}})\right]} \operatorname{Mass}\left(\Lambda_{\widetilde{x}}\right)
\end{aligned}
$$

Note that $G_{\widetilde{x}}(\widehat{\mathbb{Z}})$ and $\varphi^{*} G_{x}(\widehat{\mathbb{Z}})$ differ only at $p$. By [24, Proposition 4.8], every endomorphism of $X\left[p^{\infty}\right]$ lifts uniquely to an endomorphism of $\widetilde{X}\left[p^{\infty}\right]$. This shows the injectivity of the map $\varphi^{*}: \operatorname{End}\left(X\left[p^{\infty}\right]\right) \rightarrow \operatorname{End}\left(\widetilde{X}\left[p^{\infty}\right]\right)$. Therefore, we have the inclusion $G_{x}\left(\mathbb{Z}_{p}\right)=\operatorname{Aut}\left((X, \lambda)\left[p^{\infty}\right]\right) \hookrightarrow G_{\widetilde{x}}\left(\mathbb{Z}_{p}\right)=\operatorname{Aut}\left((\tilde{X}, \widetilde{\lambda})\left[p^{\infty}\right]\right)$ via $\varphi^{*}$ and find the first part of Equation (7).

By Dieudonné module theory, for any polarised supersingular abelian variety $(X, \lambda)$ with quasi-polarised Dieudonné module $(M,\langle\rangle$,$) , we may identify \operatorname{Aut}\left((X, \lambda)\left[p^{\infty}\right]\right)$ with $\operatorname{Aut}(M,\langle\rangle$,$) . This yields Equation (7).$

To summarise, the results of this section provide the following strategy for obtaining a mass formula for any principally polarised supersingular abelian variety:

(a) For any supersingular abelian variety $x=(X, \lambda)$, construct the minimal isogeny $\varphi:(\widetilde{X}, \widetilde{\lambda}) \rightarrow(X, \lambda)$ from a suitable superspecial abelian variety $\widetilde{x}=(\widetilde{X}, \widetilde{\lambda})$.

(b) Use Theorem 2.4 (or Corollary 2.5 if $g=3$ ) to compute $\operatorname{Mass}\left(\Lambda_{\widetilde{x}}\right)$. 
(c) Compute the local index $[\operatorname{Aut}(\widetilde{M},\langle\rangle):, \operatorname{Aut}((M,\langle\rangle)$,$] , cf. (7).$

(d) Compute $\operatorname{Mass}\left(\Lambda_{x}\right)$, i.e., $\operatorname{compare} \operatorname{Mass}\left(\Lambda_{\widetilde{x}}\right)$ and $\operatorname{Mass}\left(\Lambda_{x}\right)$ by applying Proposition 2.12.

We will carry out these steps, in particular Step (c), in the next sections in the case where $g=3$. In the next section, we start by studying in detail the moduli space $\mathscr{S}_{3,1}$ of supersingular principally polarised abelian threefolds and the minimal isogenies (cf. Definition 2.11) between threefolds.

\section{Structure of the supersingular locus $\mathscr{S}_{3,1}$}

In this section we describe the supersingular locus $\mathscr{S}_{3,1}$. Its structure will be used to determine minimal isogenies, cf. Proposition 3.16. Finer structures will be introduced in order to compute the local index in Step (c) in the previous section.

\subsection{The supersingular locus $\mathscr{S}_{g, 1}$ and the mass function}

To describe the moduli space $\mathscr{S}_{3,1}$ of supersingular principally polarised abelian threefolds, we will use the framework of polarised flag type quotients (for $g=3$ ) as developed by $\mathrm{Li}$ and Oort [13], which we will briefly describe below (for any $g \geq 1$ ). Then we will introduce the stratification of $\mathscr{S}_{g, 1}$ induced by the mass values and its local analogue.

For any abelian variety $X$, denote by $P(X)$ the set of isomorphism classes of principal polarisations on $X$.

Let $E / \mathbb{F}_{p^{2}}$ be a supersingular elliptic curve whose Frobenius endomorphism is $\pi_{E}=$ $-p$ and denote $E_{k}=E \otimes_{\mathbb{F}_{p^{2}}} k$. Since every polarisation on $E_{k}^{g}$ is defined over $\mathbb{F}_{p^{2}}$, we may identify $P\left(E_{k}^{g}\right)$ with $P\left(E^{g}\right)$. Recall that an $\alpha$-group of rank $r$ over an $\mathbb{F}_{p}$-scheme $S$ is a finite flat group scheme over $S$ which is Zariski-locally isomorphic to $\alpha_{p}^{r}$. For a scheme $X$ over $S$, put $X^{(p)}:=X \times_{S, F_{S}} S$, where $F_{S}: S \rightarrow S$ denotes the absolute Frobenius morphism on $S$, and denote by $F_{X / S}: X \rightarrow X^{(p)}$ the relative Frobenius morphism.

For each integer $i \geq 0$, let $P\left(E^{g}, i\right)$ be the set of isomorphism classes of polarisations $\lambda$ on $E^{g}$ such that ker $\lambda=E\left[\mathrm{~F}^{i}\right]$ with $\mathrm{F}=F_{E / \mathbb{F}_{p^{2}}}$ and set $P^{*}\left(E^{g}\right):=P\left(E^{g}, g-1\right)$. The map $\lambda \mapsto p^{\lfloor(g-1) / 2\rfloor} \lambda$ gives a bijection $P\left(E^{g}\right) \stackrel{\sim}{\longrightarrow} P^{*}\left(E^{g}\right)$ if $g$ is odd and $P\left(E^{g}, 1\right) \stackrel{\sim}{\longrightarrow} P^{*}\left(E^{g}\right)$ otherwise. Moreover, the map $\lambda \mapsto\left(E_{k}^{g}, \lambda\right)$ gives a bijection $P\left(E^{g}\right) \stackrel{\sim}{\longrightarrow} \Lambda_{g, 1}$ when $g$ is odd and $P\left(E^{g}, 1\right) \stackrel{\sim}{\longrightarrow} \Lambda_{g, p^{c}}$ when $g=2 c$ is even. Thus,

$$
P^{*}\left(E^{g}\right) \simeq \begin{cases}\Lambda_{g, 1}, & \text { if } g \text { is odd } \\ \Lambda_{g, p^{c}}, & \text { if } g=2 c \text { is even }\end{cases}
$$

It is known that $\left|\Lambda_{g, 1}\right|=H_{g}(p, 1)$ for any positive integer $g$ and $\left|\Lambda_{g, p^{c}}\right|=H_{g}(1, p)$ for any even positive integer $g=2 c$, where $H_{g}(p, 1)$ (resp. $\left.H_{g}(1, p)\right)$ is the class number of principal genus (resp. the non-principal genus); see [13] for details. 
Definition 3.1. (cf. [13, Section 3])

(1) Let $g \geq 1$ be an integer. For any $\mu \in P^{*}\left(E^{g}\right)$, a $g$-dimensional polarised flag type quotient (PFTQ) with respect to $\mu$ is a chain of $g$-dimensional polarised abelian schemes over a base $\mathbb{F}_{p^{2} \text {-scheme } S}$

$$
\left(Y_{\bullet}, \rho_{\bullet}\right):\left(Y_{g-1}, \lambda_{g-1}\right) \stackrel{\rho_{g-1}}{\longrightarrow}\left(Y_{g-2}, \lambda_{g-2}\right) \stackrel{\rho_{g-2}}{\longrightarrow} \cdots \stackrel{\rho_{2}}{\longrightarrow}\left(Y_{1}, \lambda_{1}\right) \stackrel{\rho_{1}}{\longrightarrow}\left(Y_{0}, \lambda_{0}\right),
$$

such that:

(i) $\left(Y_{g-1}, \lambda_{g-1}\right)=\left(E^{g}, \mu\right) \times_{\text {Spec } \mathbb{F}_{p^{2}}} S$;

(ii) $\operatorname{ker}\left(\rho_{i}\right)$ is an $\alpha$-group of rank $i$ for $1 \leq i \leq g-1$;

(iii) $\operatorname{ker}\left(\lambda_{i}\right) \subseteq \operatorname{ker}\left(\mathrm{V}^{j} \circ \mathrm{F}^{i-j}\right)$ for $0 \leq i \leq g-1$ and $0 \leq j \leq\lfloor i / 2\rfloor$, where $\mathrm{F}=$ $F_{Y_{i} / S}: Y_{i} \rightarrow Y_{i}^{(p)}$ and $\mathrm{V}=V_{Y_{i} / S}: Y_{i}^{(p)} \rightarrow Y_{i}$ are the relative Frobenius and Verschiebung morphisms, respectively.

In particular, $\lambda_{0}$ is a principal polarisation on $Y_{0}$. An isomorphism of $g$-dimensional polarised flag type quotients is a chain of isomorphisms $\left(\alpha_{i}\right)_{0 \leq i \leq g-1}$ of polarised abelian varieties such that $\alpha_{g-1}=\mathrm{id}_{Y_{g-1}}$.

(2) A $g$-dimensional polarised flag type quotient $\left(Y_{\bullet}, \rho_{\bullet}\right)$ is said to be rigid if

$$
\operatorname{ker}\left(Y_{g-1} \rightarrow Y_{i}\right)=\operatorname{ker}\left(Y_{g-1} \rightarrow Y_{0}\right) \cap Y_{g-1}\left[\mathrm{~F}^{g-1-i}\right], \quad \text { for } 1 \leq i \leq g-1,
$$

where $Y_{g-1}\left[\mathrm{~F}^{g-1-i}\right]:=\operatorname{ker}\left(\mathrm{F}^{g-1-i}: Y_{g-1} \rightarrow Y_{g-1}^{\left(p^{g-1-i}\right)}\right)$.

(3) Let $\mathscr{P}_{g, \mu}$ (resp. $\mathscr{P}_{g, \mu}^{\prime}$ ) denote the moduli space over $\mathbb{F}_{p^{2}}$ of $g$-dimensional (resp. rigid) polarised flag type quotients with respect to $\mu$.

Clearly, each member $Y_{i}$ of $\left(Y_{\bullet}, \rho_{\bullet}\right)$ is a supersingular abelian variety.

Definition 3.2. For an abelian variety $X$ over $k$, its $a$-number is defined as

$$
a(X):=\operatorname{dim}_{k} \operatorname{Hom}\left(\alpha_{p}, X\right) .
$$

The $a$-number of a Dieudonné module $M$ over $k$ is defined as $a(M):=\operatorname{dim}(M /(\mathrm{F}, \mathrm{V}) M)$. If $M$ is the Dieudonné module of $X$, then $a(M)=a(X)$. When $x \in \mathscr{P}_{g, \mu}$ corresponds to a polarised flag type quotient $\left(Y_{g-1}, \lambda_{g-1}\right) \rightarrow \cdots \rightarrow\left(Y_{1}, \lambda_{1}\right) \rightarrow\left(Y_{0}, \lambda_{0}\right)$, we say that its $a$-number is $a(x)=a\left(Y_{0}\right)$.

According to $\left[13\right.$, Lemma 3.7], $\mathscr{P}_{g, \mu}$ is a projective scheme over $\mathbb{F}_{p^{2}}$ and $\mathscr{P}_{g, \mu}^{\prime} \subset \mathscr{P}_{g, \mu}$ is an open subscheme. Thus, $\mathscr{P}_{g, \mu}^{\prime}$ a quasi-projective scheme over $\mathbb{F}_{p^{2}}$. The projection to the last member gives a proper $\overline{\mathbb{F}}_{p}$-morphism

$$
\begin{aligned}
\operatorname{pr}_{0}: \mathscr{P}_{g, \mu, \overline{\mathbb{F}}_{p}} & \rightarrow \mathscr{S}_{g, 1}, \\
\left(Y_{\bullet}, \rho_{\bullet}\right) & \mapsto\left(Y_{0}, \lambda_{0}\right) .
\end{aligned}
$$


Theorem 3.3 (Li-Oort).

(1) The natural morphism

$$
\operatorname{pr}_{0}: \coprod_{\mu \in P^{*}\left(E^{g}\right)} \mathscr{P}_{g, \mu, \overline{\mathbb{F}}_{p}} \rightarrow \mathscr{S}_{g, 1}
$$

is quasi-finite and surjective.

(2) For every $\mu \in P^{*}\left(E^{g}\right)$, the scheme $\mathscr{P}_{g, \mu}^{\prime}$ is non-singular and geometrically irreducible of dimension $\left\lfloor g^{2} / 4\right\rfloor$. Moreover, the a-number 1 locus $\mathscr{P}_{g, \mu}^{\prime}(a=1)$ is open and dense in $\mathscr{P}_{g, \mu}^{\prime}$.

(3) The morphism $\mathrm{pr}_{0}$ induces a surjective birational morphism

$$
\operatorname{pr}_{0}: \coprod_{\mu \in P^{*}\left(E^{g}\right)} \mathscr{P}_{g, \mu, \overline{\mathbb{F}}_{p}}^{\prime} / G_{\mu} \rightarrow \mathscr{S}_{g, 1}
$$

where $G_{\mu}:=\operatorname{Aut}\left(E^{g}, \mu\right)$ is the automorphism group of $\left(E^{g}, \mu\right)$. Moreover, it induces an isomorphism on the a-number 1 loci:

$$
\operatorname{pr}_{0}: \coprod_{\mu \in P^{*}\left(E^{g}\right)} \mathscr{P}_{g, \mu, \overline{\mathbb{F}}_{p}}^{\prime}(a=1) / G_{\mu} \stackrel{\sim}{\longrightarrow} \mathscr{S}_{g, 1}(a=1) .
$$

(4) The supersingular locus $\mathscr{S}_{g, 1}$ is equidimensional of dimension $\left\lfloor g^{2} / 4\right\rfloor$. The a-number 1 locus $\mathscr{S}_{g, 1}(a=1)$ is open and dense in $\mathscr{S}_{g, 1}$. It has

$$
\begin{cases}H_{g}(p, 1), & \text { for odd integer } g \\ H_{g}(1, p), & \text { for even integer } g\end{cases}
$$

geometrically irreducible components.

Proof. See [13, Section 4].

Note that $\mathscr{P}_{3, \mu}^{\prime} \subset \mathscr{P}_{3, \mu}$ is dense, while for general $g$ the open subscheme $\mathscr{P}_{g, \mu}^{\prime} \subset \mathscr{P}_{g, \mu}$ is no longer dense, cf. [13, Section 9.6].

\section{Definition 3.4.}

(1) Let $k$ be an algebraically closed field of characteristic $p>0$ and let

$$
\text { Mass : } \mathscr{S}_{g, 1}(k) \rightarrow \mathbb{Q}, \quad x \mapsto \operatorname{Mass}(x):=\operatorname{Mass}\left(\Lambda_{x}\right)
$$

be the mass function. For each mass value $r \in \mathbb{Q}$, i.e. $r=\operatorname{Mass}(x)$ for some point $x \in \mathscr{S}_{g, 1}(k)$, define a subset 


$$
\mathscr{S}_{g, 1, r}:=\left\{x \in \mathscr{S}_{g, 1}(k): \operatorname{Mass}(x)=r\right\}
$$

Then we have a decomposition of the supersingular locus into subsets

$$
\mathscr{S}_{g, 1}(k)=\coprod_{r} \mathscr{S}_{g, 1, r}
$$

where $r$ runs through all mass values. Each subset $\mathscr{S}_{g, 1, r}$ is called the mass stratum with mass value $r$, and the decomposition (14) is called the mass stratification of $\mathscr{S}_{g, 1}(k)$.

(2) For each $\mu \in P^{*}\left(E^{g}\right)$, consider the pull-back of the mass function on $\mathscr{S}_{g, 1}(k)$ by $\operatorname{pr}_{0}$. We obtain the mass function on $\mathscr{P}_{g, \mu}(k)$ :

$$
\operatorname{Mass}: \mathscr{P}_{g, \mu}(k) \rightarrow \mathbb{Q}, \quad y \mapsto \operatorname{Mass}(y):=\operatorname{Mass}\left(\Lambda_{\operatorname{pr}_{0}(y)}\right)
$$

Similarly, we define the mass stratum $\mathscr{P}_{g, \mu, r}$ for each mass value $r \in \mathbb{Q}$ as in (13) and obtain a decomposition of $\mathscr{P}_{g, \mu}(k)$ into mass strata:

$$
\mathscr{P}_{g, \mu}(k)=\coprod_{r} \mathscr{P}_{g, \mu, r},
$$

called the mass stratification of $\mathscr{P}_{g, \mu}(k)$.

When $g=1$, the supersingular locus $\mathscr{S}_{1,1}$ consists of one mass stratum. When $g=2$, there are three mass strata: one stratum with $a$-number 2 and two strata with $a$-number 1. Each mass stratum is a locally closed subset and the collection of mass strata satisfies the stratification property, namely, the closure of each stratum is the union of some strata cf. [27]. When $g=3$, we will see again from our computation that each mass stratum is a locally closed subset on both $\mathscr{P}_{3, \mu}$ and $\mathscr{S}_{3,1}$. However, the collection of mass strata does not satisfy the stratification property on $\mathscr{P}_{3, \mu}$ (because the structure morphism $\pi: \mathscr{P}_{3, \mu} \rightarrow C$ constructed in Proposition 3.7 admits a section $T$, which will be formally introduced in Definition 3.14) but it does on its open dense subscheme $\mathscr{P}_{3, \mu}^{\prime}=\mathscr{P}_{3, \mu}-T$. We expect that every mass stratum is a locally closed subset for general $\mathrm{g}$. The mass stratification encodes arithmetic information (automorphism groups and endomorphism rings) of supersingular abelian varieties. For example, we will see in Section 6 that the automorphism groups of supersingular abelian threefolds jump only when the objects cross different mass strata. Since arithmetic properties generally do not respect geometric properties, we are less optimistic that the collection of mass strata of $\mathscr{P}_{g, \mu}^{\prime}$ satisfies the stratification property.

Now we introduce a local analogue of the mass stratification where the underlying space $\mathscr{S}_{g, 1}$ is replaced with the moduli space of supersingular $p$-divisible groups, namely, the supersingular Rapoport-Zink space.

Fix a $g$-dimensional principally polarised superspecial abelian variety $x_{0}=\left(X_{0}, \lambda_{X_{0}}\right)$ over $\overline{\mathbb{F}}_{p}$, and let $\underline{\mathbf{X}}_{0}=\left(\mathbf{X}_{0}, \lambda_{\mathbf{X}_{0}}\right)=\left(X_{0}, \lambda_{X_{0}}\right)\left[p^{\infty}\right]$ be the associated principally polarised 
p-divisible group. Let $\mathscr{M}_{\overline{\mathbb{F}}_{p}}^{0}$ be the Rapoport-Zink space over $\overline{\mathbb{F}}_{p}$ classifying principally polarised quasi-isogenies of $\left(\mathbf{X}_{0}, \lambda \mathbf{X}_{0}\right)$ of height 0 . For each $\overline{\mathbb{F}}_{p}$-scheme $S, \mathscr{M}_{\overline{\mathbb{F}}_{p}}^{0}(S)$ is the set of isomorphism classes of pairs $(\underline{\mathbf{X}}, \rho)_{S}$, where

(i) $\underline{\mathbf{X}}=\left(\mathbf{X}, \lambda_{\mathbf{X}}\right)$ is a principally polarised $p$-divisible group over $S$;

(ii) $\rho: \mathbf{X}_{0} \rightarrow \mathbf{X}$ is a quasi-isogeny over $S$ such that $\rho^{*} \lambda \mathbf{X}=\lambda_{\mathbf{X}_{0}}$.

Two pairs $\left(\underline{\mathbf{X}}_{1}, \rho_{1}\right)$ and $\left(\underline{\mathbf{X}}_{2}, \rho_{2}\right)$ are isomorphic if there exists an isomorphism $\alpha: \mathbf{X}_{1} \stackrel{\sim}{\longrightarrow}$ $\mathbf{X}_{2}$ such that $\alpha \circ \rho_{1}=\rho_{2}$. One easily sees $\alpha^{*} \lambda_{\mathbf{X}_{2}}=\lambda_{\mathbf{X}_{1}}$. The Rapoport-Zink space $\mathscr{M}_{\overline{\mathbb{F}}_{p}}^{0}$ is a scheme locally of finite type over $\overline{\mathbb{F}}_{p}$, cf. [18, Theorem 3.25 and Corollary 2.29].

Let $G_{\underline{\mathbf{X}}_{0}}$ be the automorphism group scheme of $\underline{\mathbf{X}}_{0}$ over $\mathbb{Z}_{p}$. The group $G_{\underline{\mathbf{X}}_{0}}\left(\mathbb{Q}_{p}\right)$ of $\mathbb{Q}_{p}$-valued points consists of polarised quasi-self-isogenies of $\underline{\mathbf{X}}_{0}$ over $k$; it is a locally compact topological group. Choose a Haar measure on $G_{\underline{\mathbf{x}}_{0}}\left(\mathbb{Q}_{p}\right)$ with volume one on the maximal open compact subgroup $G_{\underline{\mathbf{x}}_{0}}\left(\mathbb{Z}_{p}\right)=\operatorname{Aut}\left(\underline{\mathbf{X}}_{0}\right)$. For each $k$-valued point $\mathbf{x}=(\underline{\mathbf{X}}, \rho) \in \mathscr{M}_{\overline{\mathbb{F}}_{p}}^{0}(k)$, we may regard its automorphism group $\operatorname{Aut}(\underline{\mathbf{X}})$ as an open compact subgroup of $G_{\underline{\mathbf{x}}_{0}}\left(\mathbb{Q}_{p}\right)$ by inclusion:

$$
\rho^{*}: \operatorname{Aut}(\underline{\mathbf{X}}) \hookrightarrow G_{\underline{\mathbf{X}}_{0}}\left(\mathbb{Q}_{p}\right), \quad h \mapsto \rho^{-1} \circ h \circ \rho .
$$

Definition 3.5. Let the notation be as above. Define a function on $\mathscr{M}_{\overline{\mathbb{F}}_{p}}^{0}(k)$ by

$$
v: \mathscr{M}_{\overline{\mathbb{F}}_{p}}^{0}(k) \rightarrow \mathbb{Q}, \quad \mathbf{x}=(\underline{\mathbf{X}}, \rho) \mapsto v(\mathbf{x}):=\operatorname{vol}\left(\rho^{*}(\operatorname{Aut}(\underline{\mathbf{X}}))\right)^{-1}
$$

For each $v$-value $r \in \mathbb{Q}$, that is, $r=v(\mathbf{x})$ for some $\mathbf{x} \in \mathscr{M}_{\overline{\mathbb{F}}_{p}}^{0}(k)$, consider the subset

$$
\mathscr{M}_{r}^{0}:=\left\{x \in \mathscr{M}_{\overline{\mathbb{F}}_{p}}^{0}(k): v(\mathbf{x})=r\right\},
$$

for which the function $v$ takes value $r$, called the $v$-stratum with $v$-value $r$. The RapoportZink space then decomposes in subsets:

$$
\mathscr{M}_{\overline{\mathbb{F}}_{p}}^{0}(k)=\coprod_{r} \mathscr{M}_{r}^{0}
$$

where $r$ runs through all $v$-values in $\mathbb{Q}$, called the $v$-stratification of $\mathscr{M}_{\overline{\mathbb{F}}_{p}}^{0}(k)$. Observe that the collection of $v$-strata is independent of the choice of the Haar measure on $G_{\mathbf{x}_{0}}\left(\mathbb{Q}_{p}\right)$ as the function $v^{\prime}$ associated to a different Haar measure is just a multiple of $v$ by a scalar.

Let

$$
\widetilde{\pi}: \mathscr{M}_{\overline{\mathbb{F}}_{p}}^{0} \rightarrow \mathscr{S}_{g, 1}
$$

be the Rapoport-Zink uniformisation morphism, cf. [18, 6.13]. 
Proposition 3.6. The stratification of $\mathscr{M}_{\overline{\mathbb{F}}_{p}}^{0}(k)$ obtained by the pull-back of the mass stratification of $\mathscr{S}_{g, 1}(k)$ by $\widetilde{\pi}$ coincides with the $v$-stratification.

Proof. We compare the functions $v$ and $\tilde{\pi}^{*}$ Mass $=$ Mass $\circ \widetilde{\pi}$. Let $\mathbf{x}=(\underline{\mathbf{X}}, \rho)$ be a $k$ valued point in $\mathscr{M}_{\overline{\mathbb{F}}_{p}}^{0}(k)$. Then $\mathbf{x}$ lifts to a pair $\left(\left(X, \lambda_{X}\right), \widetilde{\rho}\right)$ of a principally polarised supersingular abelian variety $\left(X, \lambda_{X}\right)$ and a polarised quasi-isogeny $\widetilde{\rho}:\left(X_{0}, \lambda_{X_{0}}\right) \rightarrow$ $\left(X, \lambda_{X}\right)$. By the construction of $[18,6.13]$, the map $\widetilde{\pi}$ sends $\mathbf{x}$ to $x:=\left(X, \lambda_{X}\right)$. Using Proposition 2.7 and Lemma 2.9, we see that

$$
\begin{aligned}
\operatorname{Mass}(x)=\operatorname{Mass}\left(\Lambda_{x}\right) & =\frac{\operatorname{vol}\left(G_{x_{0}}\left(\mathbb{Z}_{p}\right)\right)}{\operatorname{vol}\left(\widetilde{\rho}^{*}\left(G_{x}\left(\mathbb{Z}_{p}\right)\right)\right)} \operatorname{Mass}\left(\Lambda_{x_{0}}\right) \\
& =\frac{\operatorname{Mass}\left(\Lambda_{x_{0}}\right)}{\operatorname{vol}\left(\rho^{*}\left(\operatorname{Aut}\left(\mathbf{X}, \lambda_{\mathbf{X}}\right)\right)\right)}=\operatorname{Mass}\left(x_{0}\right) \cdot v(\mathbf{x}) .
\end{aligned}
$$

Thus, $\tilde{\pi}^{*} \operatorname{Mass}(\mathbf{x})=\operatorname{Mass}\left(x_{0}\right) \cdot v(\mathbf{x})$ for $\mathbf{x} \in \mathscr{M}_{\overline{\mathbb{F}}_{p}}^{0}(k)$ and the assertion follows.

\subsection{The structure of $\mathscr{S}_{3,1}$}

Hereafter we will only treat the case where $g=3$. For brevity, we write $\mathscr{P}_{\mu}$ and $\mathscr{P}_{\mu}^{\prime}$ for $\mathscr{P}_{3, \mu}$ and $\mathscr{P}_{3, \mu}^{\prime}$, respectively. Roughly speaking, Equation (9) says that each $\mathscr{P}_{\mu}$ approximates an irreducible component of the supersingular locus $\mathscr{S}_{3,1}$. More precisely, one can show the following structure results; for more details, we refer to [13, Sections 9.39.4]. Let $C \subseteq \mathbb{P}^{2}$ be the Fermat curve defined by the equation $X_{1}^{p+1}+X_{2}^{p+1}+X_{3}^{p+1}=0$.

Proposition 3.7. The Fermat curve $C$ can be interpreted as the classifying space of isogenies $\left(Y_{2}, \lambda_{2}\right) \rightarrow\left(Y_{1}, \lambda_{1}\right)$ whose kernel is locally isomorphic to $\alpha_{p}^{2}$. Moreover, there is an isomorphism $\mathscr{P}_{\mu} \simeq \mathbb{P}_{C}(\mathscr{O}(-1) \oplus \mathscr{O}(1))$ for which the structure morphism $\pi: \mathbb{P}_{C}(\mathscr{O}(-1) \oplus \mathscr{O}(1)) \rightarrow C$ corresponds to the forgetful map $\left(\left(Y_{2}, \lambda_{2}\right) \rightarrow\left(Y_{1}, \lambda_{1}\right) \rightarrow\right.$ $\left.\left(Y_{0}, \lambda_{0}\right)\right) \mapsto\left(\left(Y_{2}, \lambda_{2}\right) \rightarrow\left(Y_{1}, \lambda_{1}\right)\right)$

Proof. Let $M_{2}$ be the polarised contravariant Dieudonné module of $Y_{2}$. Choosing an isogeny $\rho_{2}$ from $E_{k}^{3}$ such that $\operatorname{ker}\left(\rho_{2}\right) \simeq \alpha_{p}^{2}$ is equivalent to choosing a surjection of Dieudonné modules $M_{2} \rightarrow k^{2}$. Since Frobenius $\mathrm{F}$ and Verschiebung $\mathrm{V}$ act as zero on $k^{2}$, this is further equivalent to choosing a one-dimensional subspace of the three-dimensional (since $\left.a\left(Y_{2}\right)=3\right) k$-vector space $M_{2} /(\mathrm{F}, \mathrm{V}) M_{2}$ which corresponds to a point $\left(t_{1}: t_{2}\right.$ : $\left.t_{3}\right) \in \mathbb{P}^{2}=\mathbb{P}\left(\left(M_{2} /(\mathrm{F}, \mathrm{V}) M_{2}\right)^{*}\right)$.

The polarisation $\lambda_{2}=p \mu$ descends to a polarisation $\lambda_{1}$ on $Y_{1}$ through such $\rho_{2}$, and the condition $\operatorname{ker}\left(\lambda_{1}\right) \subseteq Y_{1}[\mathrm{~F}]$ is equivalent to the condition

$$
t_{1}^{p+1}+t_{2}^{p+1}+t_{3}^{p+1}=0
$$

which describes the Fermat curve $C$ of degree $p+1$ in $\mathbb{P}^{2}$. For precise computations, we refer to [12]. 
Let $M_{1}$ be the polarised Dieudonné module of $Y_{1}$ : the polarisation $\lambda_{1}$ induces a quasipolarisation $D\left(\lambda_{1}\right): M_{1}^{\vee} \rightarrow M_{1}$, and we regard $M_{1}^{\vee}$ as an submodule of $M_{1}$ under this injection. One has the inclusions $M_{1}^{\vee} \subset \mathrm{V} M_{2} \subset M_{1}$ as $\vee M_{2}$ is self-dual with respect to the quasi-polarisation induced by $\lambda_{1}$ and $\mathrm{V} M_{2}=(\mathrm{F}, \mathrm{V}) M_{2} \subset M_{1}$. Choosing a second isogeny $\left(Y_{1}, \lambda_{1}\right) \rightarrow\left(Y_{0}, \lambda_{0}\right)$ is equivalent to choosing a one-dimensional subspace of the twodimensional vector space $M_{1} / M_{1}^{\vee}$. Thus each fibre of the structure morphism $\pi: \mathscr{P}_{\mu} \rightarrow C$ is isomorphic to $\mathbb{P}\left(\left(M_{1} / M_{1}^{\vee}\right)^{*}\right) \simeq \mathbb{P}^{1}$ and this fibration corresponds to a rank two vector bundle $\mathscr{V}$ on $C$. The canonical one-dimensional space (F, $\mathrm{V}) M_{2} / M_{1}^{\vee} \subseteq M_{1} / M_{1}^{\vee}$ defines a section $s$ of $\pi: \mathscr{P}_{\mu} \rightarrow C$ and corresponds to a surjection $\mathscr{V} \rightarrow \mathscr{O}(-1)$. By the duality of polarisations, we see that $\mathscr{V}$ is an extension of $\mathscr{O}(-1)$ by $\mathscr{O}(1)$ and this extension splits.

Since the Fermat curve $C$ is a smooth plane curve of degree $p+1$, its genus is equal to $p(p-1) / 2$. Let $U_{3}\left(\mathbb{F}_{p}\right) \subseteq \mathrm{GL}_{3}\left(\mathbb{F}_{p^{2}}\right)$ denote the unitary subgroup consisting of matrices $A$ such that $A^{T} A^{(p)}=\mathbb{I}_{3}$. We see that for each $A \in U_{3}\left(\mathbb{F}_{p}\right)$ and $t \in C$, the matrix multiplication $A \cdot t^{T}$ lies in $C$. This gives a left action of $U_{3}\left(\mathbb{F}_{p}\right)$ on the curve $C$. It is known that $\left|U_{3}\left(\mathbb{F}_{p}\right)\right|=p^{3}(p+1)\left(p^{2}-1\right)\left(p^{3}+1\right)$.

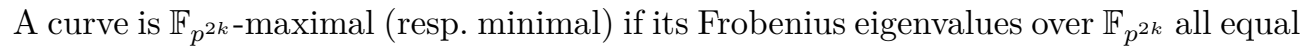
$-p^{k}$ (resp. $p^{k}$ ). From the well-understood behaviour of Frobenius eigenvalues under field extensions we then derive the following lemma.

Lemma 3.8. We have $\left|C\left(\mathbb{F}_{p^{2}}\right)\right|=p^{3}+1$. Thus, it is $\mathbb{F}_{p^{2}}$-maximal and hence $\mathbb{F}_{p^{4}-\text { minimal. }}$ Moreover, we have $C\left(\mathbb{F}_{p^{2}}\right)=C\left(\mathbb{F}_{p^{4}}\right)$. Furthermore, we have

$$
\left|C\left(\mathbb{F}_{p^{2 i}}\right)\right|= \begin{cases}p^{2 i}+p^{i+2}-p^{i+1}+1 & \text { if } i \text { is odd } \\ p^{2 i}-p^{i+2}+p^{i+1}+1 & \text { if } i \text { is even }\end{cases}
$$

Proof. For each $t=\left(t_{i}\right) \in C\left(\mathbb{F}_{p^{2}}\right)$, let $s_{i}=t_{i}^{p+1}$. Then $s_{i} \in \mathbb{F}_{p}$ and $s_{1}+s_{2}+s_{3}=0$. So there are $p+1$ points $\left(s_{i}\right)$ in $\mathbb{P}^{1}\left(\mathbb{F}_{p}\right)$. For each point $\left(s_{i}\right)$, there are $p+1\left(\operatorname{resp} .(p+1)^{2}\right)$ points $\left(t_{i}\right)$ over $\left(s_{i}\right)$ if some of the $s_{i}$ are zero (resp. otherwise); there are 3 points $\left(s_{i}\right)$ with $s_{i}=0$ for some $i$. Thus,

$$
\left|C\left(\mathbb{F}_{p^{2}}\right)\right|=(p+1-3)(p+1)^{2}+3(p+1)=p^{3}+1
$$

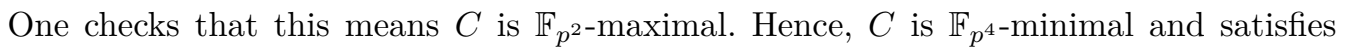
$\left|C\left(\mathbb{F}_{p^{4}}\right)\right|=p^{3}+1$. Since $C$ is $\mathbb{F}_{p^{2 i}}$-maximal (resp. $\mathbb{F}_{p^{2 i}}$-minimal) if $i$ is odd (resp. even), the formula (17) follows immediately.

Lemma 3.9. Let $t=\left(t_{1}: t_{2}: t_{3}\right) \in C(k)$. Then $t \in C\left(\mathbb{F}_{p^{2}}\right)$ if and only if $t_{1}, t_{2}, t_{3}$ are linearly dependent over $\mathbb{F}_{p^{2}}$.

Proof. See [16, Lemma 2.1]. Alternatively, we give the following independent proof: 
The forward implication is immediate, so we will only show the reverse implication. Assume $t_{1}, t_{2}, t_{3}$ are linearly dependent over $\mathbb{F}_{p^{2}}$. Then the vectors $\left(t_{i}, t_{i}^{p^{2}}, t_{i}^{p^{4}}\right)$ for $i=$ $1,2,3$ are $k$-linearly dependent. If $\left(t_{i}, t_{i}^{p^{2}}, t_{i}^{p^{4}}\right)$ for $i=2,3$ are linearly independent, then there exist $a, b \in k$ such that $t_{i}=a t_{i}^{p^{2}}+b t_{i}^{p^{4}}$ for $i=1,2,3$. If they are linearly dependent, then there exists $a^{\prime} \in k$ such that $t_{i}^{p^{2}}=a^{\prime} t_{i}^{p^{4}}$ for $i=1,2,3$ and hence $t_{i}=a t_{i}^{p^{2}}$ with $a^{p^{2}}=a^{\prime}$. Therefore, there exist $a, b \in k$ such that $t_{i}=a t_{i}^{p^{2}}+b t_{i}^{p^{4}}$ for $i=1,2,3$ in either case. Substituting this into the defining equation of $C$, we obtain

$$
a^{p+1} \sum_{i=1}^{3} t_{i}^{p^{2}+p^{3}}+a b^{p} \sum_{i=1}^{3} t_{i}^{p^{2}+p^{5}}+a^{p} b \sum_{i=1}^{3} t_{i}^{p^{3}+p^{4}}+b^{p+1} \sum_{i=1}^{3} t_{i}^{p^{4}+p^{5}}=0 .
$$

Again using the defining equation of $C$, we see that the first, third, and fourth terms vanish, so that also $a b^{p} \sum_{i=1}^{3} t_{i}^{p^{2}+p^{5}}=a b^{p}\left(\sum_{i=1}^{3} t_{i}^{p^{3}+1}\right)^{p^{2}}=0$. If $a=0$ then the point $t=\left(t_{1}: t_{2}: t_{3}\right)$ is defined over $\mathbb{F}_{p^{4}}$ and hence, by Lemma 3.8, it is defined over $\mathbb{F}_{p^{2}}$. If $b=0$, then $t$ is defined over $\mathbb{F}_{p^{2}}$ as well. So we may assume that $\sum_{i=1}^{3} t_{i}^{p^{3}+1}=0$. Let $Z:=V\left(X_{1}^{p^{3}+1}+X_{2}^{p^{3}+1}+X_{3}^{p^{3}+1}\right)$ be the Fermat curve of degree $p^{3}+1$. Then $t \in C \cap Z$. The intersection number of $C$ and $Z$ is $(p+1)\left(p^{3}+1\right)$ and each point of $C\left(\mathbb{F}_{p^{2}}\right)$ is in $C \cap Z$. Since $\left|C\left(\mathbb{F}_{p^{2}}\right)\right|=p^{3}+1$ by Lemma 3.8, it is enough to show that for each point $s \in C\left(\mathbb{F}_{p^{2}}\right)$, the local multiplicity of $C$ and $Z$ at $s$ is $p+1$. Since the unitary group $U_{3}\left(\mathbb{F}_{p}\right)$ acts transitively on $C\left(\mathbb{F}_{p^{2}}\right)$, we may assume that $s=(\zeta: 0: 1)$ where $\zeta^{p+1}=-1$. With local coordinates $v=X_{1}-\zeta$ and $w=X_{2}$, the respective equations for $C$ and $Z$ at $y$ become $v^{p+1}+\zeta v^{p}+\zeta v+w^{p+1}$ and $v^{p^{3}+1}+\zeta v^{p^{3}}+\zeta^{p} v+w^{p^{3}+1}$. Now we may read off that the local multiplicity, i.e., the valuation of $v$ at $s$, is $p+1$, as required.

We will denote $C^{0}:=C \backslash C\left(\mathbb{F}_{p^{2}}\right)$. Slightly abusively, we will tacitly switch between the notations $\left(t_{1}, t_{2}, t_{3}\right)$ and $\left(t_{1}: t_{2}: t_{3}\right)$. For later use, we define the following:

Definition 3.10. For $t=\left(t_{1}, t_{2}, t_{3}\right) \in k^{3}$ (viewed as a column vector), let

$$
\operatorname{End}(t)=\left\{A \in \operatorname{Mat}_{3}\left(\mathbb{F}_{p^{2}}\right): A \cdot t \in k \cdot t\right\}
$$

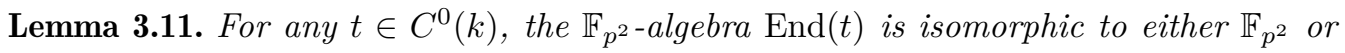
$\mathbb{F}_{p^{6}}$.

Proof. For any $A \in \operatorname{End}(t)$, we have $A \cdot t=\alpha_{A} t$ for some $\alpha_{A} \in k$. The map

$$
\begin{aligned}
\operatorname{End}(t) & \rightarrow k \\
A & \mapsto \alpha_{A}
\end{aligned}
$$

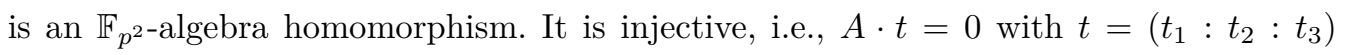
implies that $A=0$, since the $t_{i}$ are linearly independent over $\mathbb{F}_{p^{2}}$ by Lemma 3.9. Hence, $\operatorname{End}(t)$ is a finite field extension of $\mathbb{F}_{p^{2}}$. Since $\operatorname{End}(t) \subseteq \operatorname{Mat}_{3}\left(\mathbb{F}_{p^{2}}\right)=\operatorname{End}\left(\left(\mathbb{F}_{p^{2}}\right)^{3}\right)$, we 
may regard $\left(\mathbb{F}_{p^{2}}\right)^{3}$ as a vector space over $\operatorname{End}(t)$. It follows that $\left[\operatorname{End}(t): \mathbb{F}_{p^{2}}\right] \mid 3$, as required.

Lemma 3.12. We have

$$
C M:=\left\{t \in C^{0}(k): \operatorname{End}(t) \simeq \mathbb{F}_{p^{6}}\right\}=C^{0}\left(\mathbb{F}_{p^{6}}\right)
$$

Proof. The containment $\left\{t \in C^{0}(k): \operatorname{End}(t) \simeq \mathbb{F}_{p^{6}}\right\} \subseteq C^{0}\left(\mathbb{F}_{p^{6}}\right)$ is immediate, because $t$ is an eigenvector of a matrix in $\operatorname{Mat}_{3}\left(\mathbb{F}_{p^{2}}\right)$ and can be solved over the ground field $\mathbb{F}_{p^{6}}$. We will now prove the reverse containment.

For each $t \in C^{0}\left(\mathbb{F}_{p^{6}}\right)$, we construct for each element $\alpha \in \mathbb{F}_{p^{6}}$ a matrix $A \in \operatorname{Mat}_{3}\left(\mathbb{F}_{p^{6}}\right)$ as follows

$$
A=A_{\alpha}:=\left(t, t^{\left(p^{2}\right)}, t^{\left(p^{4}\right)}\right) \cdot \operatorname{diag}\left(\alpha, \alpha^{p^{2}}, \alpha^{p^{4}}\right) \cdot\left(t, t^{\left(p^{2}\right)}, t^{\left(p^{4}\right)}\right)^{-1}
$$

Since the $t_{i}$ are linearly independent over $\mathbb{F}_{p^{2}}$ by Lemma 3.9, the matrix $\left(t, t^{\left(p^{2}\right)}, t^{\left(p^{4}\right)}\right)$ is invertible. We check that

$$
\begin{aligned}
A^{\left(p^{2}\right)} & =\left(t^{\left(p^{2}\right)}, t^{\left(p^{4}\right)}, t\right) \cdot \operatorname{diag}\left(\alpha^{p^{2}}, \alpha^{p^{4}}, \alpha\right) \cdot\left(t^{\left(p^{2}\right)}, t^{\left(p^{4}\right)}, t\right)^{-1} \\
& =\left(t, t^{\left(p^{2}\right)}, t^{\left(p^{4}\right)}\right) \cdot\left(\begin{array}{lll}
0 & 0 & 1 \\
1 & 0 & 0 \\
0 & 1 & 0
\end{array}\right) \cdot \operatorname{diag}\left(\alpha^{p^{2}}, \alpha^{p^{4}}, \alpha\right) \cdot\left(\begin{array}{lll}
0 & 1 & 0 \\
0 & 0 & 1 \\
1 & 0 & 0
\end{array}\right) \cdot\left(t, t^{\left(p^{2}\right)}, t^{\left(p^{4}\right)}\right)^{-1} \\
& =A,
\end{aligned}
$$

and hence $A \in \operatorname{Mat}_{3}\left(\mathbb{F}_{p^{2}}\right)$. We also have that $A_{\alpha} \cdot t=\alpha$. Thus, the map $\alpha \in \mathbb{F}_{p^{6}} \mapsto A_{\alpha}$ gives an isomorphism $\mathbb{F}_{p^{6}} \simeq \operatorname{End}(t)$, as required.

\section{Remark 3.13.}

(1) We can also show that $U_{3}\left(\mathbb{F}_{p}\right)$ acts transitively on $C^{0}\left(\mathbb{F}_{p^{6}}\right)=C M$. The action on $C\left(\mathbb{F}_{p^{2}}\right)$ is also transitive, with stabilisers of size $p^{3}(p+1)\left(p^{2}-1\right)$; this gives another proof of the result $\left|C\left(\mathbb{F}_{p^{2}}\right)\right|=p^{3}+1$.

(2) The proof of Lemma 3.11 proves the following more general result. Let $F$ be any field contained in a field $K$ and $t_{1}, t_{2}, \ldots, t_{n}$ be a set of $F$-linearly independent elements in $K$. Put $t=\left(t_{1}, \ldots, t_{n}\right)^{T}$ and $\operatorname{End}(t):=\left\{A \in \operatorname{Mat}_{n}(F): A \cdot t \subseteq K \cdot t\right\}$. Then $\operatorname{End}(t)$ is a finite field extension of $F$ of degree dividing $n$.

Furthermore, suppose that $t_{1}, \ldots, t_{n}$ are contained a degree $n$ subextension $E$ of $F$ in $K$. Then the $F$-basis $t_{1}, \ldots, t_{n}$ of $E$ determines an $F$-algebra embedding $r: E \rightarrow$ $\operatorname{Mat}_{n}(F)$ which is characterised by $r(a) \cdot t=a t$ for every $a \in E$. Thus, $E \simeq \operatorname{End}(t)$ and $t$ is an eigenvector of a matrix in $\operatorname{Mat}_{n}(F)$. This is an abstract way of doing what is done explicitly in the second part of the proof of Lemma 3.12. 
Definition 3.14. The morphism $\pi: \mathscr{P}_{\mu} \rightarrow C$ admits a section $s$ defined as follows. For a base scheme $S$, let $\rho_{2}:\left(Y_{2}, p \mu\right) \rightarrow\left(Y_{1}, \lambda_{1}\right)$ be an object in $C(S)$. Put $\left(Y_{2}^{(p)}, \mu^{(p)}\right):=$ $(Y, \mu) \times_{S, F_{S}} S$, where $F_{S}: S \rightarrow S$ is the absolute Frobenius map. The relative Frobenius morphism $\mathrm{F}: Y_{2} \rightarrow Y_{2}^{(p)}$ gives rise to a morphism of polarised abelian schemes $\mathrm{F}$ : $\left(Y_{2}, p \mu\right) \rightarrow\left(Y_{2}^{(p)}, \mu^{(p)}\right)$. Since $\operatorname{ker}\left(\rho_{2}\right) \subseteq \operatorname{ker}(\mathrm{F})$, the morphism factors through an isogeny $\rho_{1}: Y_{1} \rightarrow Y_{2}^{(p)}$. As $\rho_{2}^{*} \rho_{1}^{*} \mu^{(p)}=\mathrm{F}^{*} \mu^{(p)}=p \mu=\rho_{2}^{*} \lambda_{1}$, we see that $\rho_{1}^{*} \mu^{(p)}=\lambda_{1}$ and thus obtain a polarised flag type quotient

$$
\left(Y_{2}, p \mu\right) \stackrel{\rho_{2}}{\longrightarrow}\left(Y_{1}, \lambda_{1}\right) \stackrel{\rho_{1}}{\longrightarrow}\left(Y_{2}^{(p)}, \mu^{(p)}\right) .
$$

This defines the section $s$, whose image will be denoted by $T$.

Recall the definition of the $a$-number from Definition 3.2. For an abelian threefold $X$ over $k$, we have $a(X) \in\{1,2,3\}$.

Proposition 3.15. Let the notation be as above.

(1) We have $\mathscr{P}_{\mu}^{\prime}=\mathscr{P}_{\mu}-T$.

(2) If $x \in T$ then we have $a(x)=3$.

(3) For any $t \in C(k)$, we have $t \in C\left(\mathbb{F}_{p^{2}}\right)$ if and only if $a(x) \geq 2$ for any $x \in \pi^{-1}(t)$.

(4) For any $x \in \mathscr{P}_{\mu}(k)$, we have $a(x)=1$ if and only if $x \notin T$ and $\pi(x) \notin C\left(\mathbb{F}_{p^{2}}\right)$.

Proof. See [13, Section 9.4].

\subsection{Minimal isogenies}

Given a polarised flag type quotient $Y_{2}=E_{k}^{3} \stackrel{\rho_{2}}{\longrightarrow} Y_{1} \stackrel{\rho_{1}}{\longrightarrow} Y_{0}=X$, the composite map $\rho_{1} \circ \rho_{2}:\left(Y_{2}, \lambda_{2}\right) \rightarrow\left(Y_{0}, \lambda_{0}\right)=(X, \lambda)$ is an isogeny from a superspecial abelian variety $Y_{2}$. Thus, this isogeny factors through the minimal isogeny of $(X, \lambda)$ :

$$
\left(Y_{2}, \lambda_{2}\right) \stackrel{\rho_{1} \circ \rho_{2}}{\longrightarrow}(\tilde{X}, \tilde{\lambda}) \stackrel{\varphi}{\longrightarrow}(X, \lambda)
$$

Since every member $(X, \lambda) \in \mathscr{S}_{3,1}(k)$ can be constructed from a polarised flag type quotient $\left(Y_{\bullet}, \rho_{\bullet}\right)$, we can construct the minimal isogeny of $(X, \lambda)$ from $\left(Y_{\bullet}, \rho_{\bullet}\right)$.

To describe the minimal isogenies for supersingular abelian threefolds in more detail, in the following proposition we separate into three cases, based on the $a$-number of the threefold.

Proposition 3.16. Let $(X, \lambda)$ be a supersingular principally polarised abelian threefold over $k$. Suppose that $(X, \lambda)$ lies in the image of $\mathscr{P}_{\mu}^{\prime}$ under the map $\mathscr{P}_{\mu}^{\prime} \rightarrow \mathscr{S}_{3,1}$ for some $\mu \in P\left(E^{3}\right)$, so that there is a unique PFTQ over $(X, \lambda)$. 
(1) If $a(X)=1$, then the associated polarised flag type quotient $\left(Y_{2}, \lambda_{2}\right) \stackrel{\rho_{2}}{\longrightarrow}\left(Y_{1}, \lambda_{1}\right) \stackrel{\rho_{1}}{\longrightarrow}$ $\left(Y_{0}, \lambda_{0}\right)=(X, \lambda)$ gives the minimal isogeny $\varphi:=\rho_{1} \circ \rho_{2}$ of degree $p^{3}$.

(2) If $a(X)=2$, then in the associated polarised flag type quotient $Y_{2}=E_{k}^{3} \rightarrow Y_{1} \rightarrow$ $Y_{0}=X$ we have $a\left(Y_{1}\right)=3$, so $Y_{1}$ is superspecial. Thus, the minimal isogeny is $\rho_{1}:\left(Y_{1}, \lambda_{1}\right) \rightarrow(X, \lambda)$ of degree $p$, where $\rho_{1}^{*} \lambda=\lambda_{1}$ satisfies $\operatorname{ker}\left(\lambda_{1}\right) \simeq \alpha_{p} \times \alpha_{p}$.

(3) If $a(X)=3$, then $X$ is superspecial. Thus, $X$ is $k$-isomorphic to $E_{k}^{3}$ and the minimal isogeny is the identity map.

Proof. (1) Let $M_{2}, M_{1}, M_{0}$ denote the Dieudonné modules of $Y_{2}, Y_{1}, Y_{0}=X$, respectively. Then $a\left(M_{2}\right)=3$. Suppose that $a\left(M_{0}\right)=1$. By Proposition 3.15, this corresponds to a point $t=\left(t_{1}: t_{2}: t_{3}\right) \notin C\left(\mathbb{F}_{p^{2}}\right)$. We claim that $a\left(M_{1}\right)=2$, which implies the statement. The Dieudonné modules satisfy the following inclusions:

$$
\begin{aligned}
& \begin{array}{ccccc}
M_{2} & \supseteq & M_{1} & \supseteq & M_{0} \\
& \supset & \text { UI } & & \text { UI }
\end{array}
\end{aligned}
$$

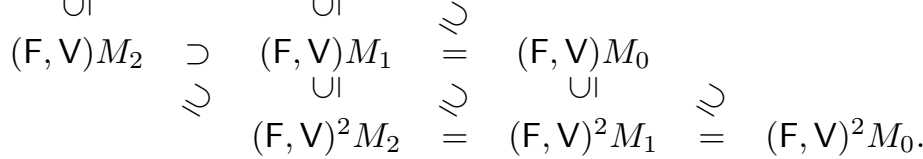

All inclusions follow from the construction of flag type quotients. For the equalities, we note the following: Since $M_{2}$ is superspecial of genus three, we have $(\mathrm{F}, \mathrm{V}) M_{2}=$ $\mathrm{F} M_{2},(\mathrm{~F}, \mathrm{~V})^{2} M_{2}=p M_{2}$, and

$$
\operatorname{dim}\left(M_{2} / F M_{2}\right)=\operatorname{dim}\left(\mathrm{F} M_{2} / p M_{2}\right)=3 .
$$

It follows from the definition of flag type quotients that $\operatorname{dim}\left(M_{1} / F M_{2}\right)=1$, so $M_{1} / F M_{2}$ is generated by one element, namely the image of $t$ (abusively again denoted $t$ ). So $(\mathrm{F}, \mathrm{V}) M_{1} / p M_{2}$ is two-dimensional and generated by the two elements $\mathrm{F} t$ and $\mathrm{V} t$, which are $k$-linearly independent since $t \notin C\left(\mathbb{F}_{p^{2}}\right)$, by Lemma 3.9. Using this, we see that

$$
\operatorname{dim}\left(\mathrm{F} M_{2} /(\mathrm{F}, \mathrm{V}) M_{1}\right)=\operatorname{dim}\left(\mathrm{F} M_{2} / p M_{2}\right)-\operatorname{dim}\left((\mathrm{F}, \mathrm{V}) M_{1} / p M_{2}\right)=1
$$

and $a\left(M_{1}\right)=\operatorname{dim}\left(M_{1} /(\mathrm{F}, \mathrm{V}) M_{1}\right)=2$, as claimed. It follows from $\operatorname{dim}\left(M_{1} / M_{0}\right)=1$ and $a\left(M_{1}\right)=2$ that $\operatorname{dim}\left(M_{0} /(\mathrm{F}, \mathrm{V}) M_{1}\right)=1$. As we have assumed that $a\left(M_{0}\right)=$ $\operatorname{dim}\left(M_{0} /(\mathrm{F}, \mathrm{V}) M_{0}\right)=1$, the latter implies the equality $(\mathrm{F}, \mathrm{V}) M_{1}=(\mathrm{F}, \mathrm{V}) M_{0}$. Since $\operatorname{dim}\left(M_{0} /(\mathrm{F}, \mathrm{V}) M_{1}\right)=1$ and $\operatorname{dim}\left(M_{0} / p M_{2}\right)=3$, one has $\left.\operatorname{dim}(\mathrm{F}, \mathrm{V}) M_{1} / p M_{2}\right)=2$. Since $t_{1}, t_{2}, t_{3}$ are $\mathbb{F}_{p^{2}}$-linearly independent by Lemma 3.9, the vectors $\mathrm{F}^{2} t, p t$ and $\mathrm{V}^{2} t$ in $\mathrm{F} M_{2} / p \mathrm{~F} M_{2}$ span a 3-dimensional subspace and hence $\operatorname{dim}\left((\mathrm{F}, \mathrm{V})^{2} M_{1} / p \mathrm{~F} M_{2}\right)=3$. This shows the equality $p M_{2}=(\mathrm{F}, \mathrm{V})^{2} M_{1}=(\mathrm{F}, \mathrm{V})^{2} M_{0}$.

Now put $\Phi:=1+\mathrm{FV}^{-1}$. We have shown that $\mathrm{V} \Phi M_{0}=(\mathrm{F}, \mathrm{V}) M_{1}$ is not superspecial and that $\Phi^{2} M_{0}=M_{2}$ is superspecial. Therefore, $M_{2}$ is the smallest superspecial Dieudonné module containing $M_{0}$. This proves that $\rho_{1} \circ \rho_{2}: Y_{2} \rightarrow X$ is the minimal isogeny. 
(2) When $a\left(M_{0}\right)=2$, this corresponds to a point $t=\left(t_{1}: t_{2}: t_{3}\right) \in C\left(\mathbb{F}_{p^{2}}\right)$. Using the notation from the previous item, we still have that $(\mathrm{F}, \mathrm{V}) M_{1} / p M_{2}$ is generated by $\mathrm{F} t$ and $\mathrm{V} t$, but since the $t_{i}$ are $\mathbb{F}_{p^{2}}$-linearly dependent, we have $\operatorname{dim}\left((\mathrm{F}, \mathrm{V}) M_{1} / p M_{2}\right)=1$, so $a\left(M_{1}\right)=3$. Since $\operatorname{ker}\left(\lambda_{1}\right) \subseteq Y_{1}[F] \simeq \alpha_{p}^{3}$, we have $\operatorname{ker}\left(\lambda_{1}\right) \simeq \alpha_{p}^{2}$, as claimed.

(3) The fact that $a(X)=3$ if and only if $X$ is superspecial is due to Oort, [15, Theorem 2].

Remark 3.17. The proof of [13, Lemma 1.8] uses the claim: If $X$ is a $g$-dimensional supersingular abelian variety with $a(X)<g$, and $X^{\prime}:=X / A(X)$, where $A(X)$ is the maximal $\alpha$-subgroup of $X$, then $a\left(X^{\prime}\right)>a(X)$.

Now take $Y_{1}$ the abelian threefold as in Proposition 3.16(1). We have computed $a\left(Y_{1}\right)=2$ and

$$
\begin{aligned}
a\left(Y_{1} / A\left(Y_{1}\right)\right) & =a\left((\mathrm{~F}, \mathrm{~V}) M_{1}\right)=\operatorname{dim}(\mathrm{F}, \mathrm{V}) M_{0} /(\mathrm{F}, \mathrm{V})^{2} M_{1} \\
& =\operatorname{dim} M_{0} /(\mathrm{F}, \mathrm{V})^{2} M_{1}-\operatorname{dim} M_{0} /(\mathrm{F}, \mathrm{V}) M_{0}=2 .
\end{aligned}
$$

This gives a counterexample to the claim.

\section{The case $a(X) \geq 2$}

Let $x=(X, \lambda) \in \mathscr{S}_{3,1}(k)$ with $a(X)=2$ and let $y \in \mathscr{P}_{\mu} \simeq \mathbb{P}_{C}^{1}(\mathscr{O}(-1) \oplus \mathscr{O}(1))$ be the point corresponding to the PFTQ over it:

$$
\left(Y_{2}, \lambda_{2}\right) \stackrel{\rho_{2}}{\longrightarrow}\left(Y_{1}, \lambda_{1}\right) \stackrel{\rho_{1}}{\longrightarrow}\left(Y_{0}, \lambda_{0}\right)=(X, \lambda)
$$

By Propositions 3.15 and 3.16, $\left(Y_{1}, \lambda_{1}\right)$ corresponds to a point $t=\left(t_{1}, t_{2}, t_{3}\right) \in C\left(\mathbb{F}_{p^{2}}\right)$ and $u \in \mathbb{P}_{t}^{1}(k):=\pi^{-1}(t)$. Moreover, $\rho_{1}:\left(Y_{1}, \lambda_{1}\right) \rightarrow(X, \lambda)$ is the minimal isogeny. Put $x_{1}=\left(Y_{1}, \lambda_{1}\right)$. Then $\Lambda_{x_{1}}=\Lambda_{3, p}$ and by Corollary 2.5 and Proposition 2.12 we have

$$
\operatorname{Mass}\left(\Lambda_{x}\right)=\frac{(p-1)\left(p^{3}+1\right)\left(p^{3}-1\right)}{2^{10} \cdot 3^{4} \cdot 5 \cdot 7} \cdot\left[\operatorname{Aut}\left(M_{1},\langle\rangle\right): \operatorname{Aut}(M,\langle,\rangle)\right]
$$

where $(M,\langle\rangle,) \subseteq\left(M_{1},\langle\rangle,\right)$ are the quasi-polarised Dieudonné modules associated to $\left(Y_{1}, \lambda_{1}\right) \rightarrow(X, \lambda)$.

Let $M_{1}^{\vee}$ denote the dual lattice of $M_{1}$ with respect to $\langle$,$\rangle . Then one has M_{1}^{\vee} \subseteq M \subseteq$ $M_{1}$ and $M / M_{1}^{\vee} \in \mathbb{P}\left(M_{1} / M_{1}^{\vee}\right)=\mathbb{P}_{t}^{1}(k)$ is a one-dimensional $k$-subspace in $M_{1} / M_{1}^{\vee}$. Since the morphism $\rho_{2}$ is defined over $\mathbb{F}_{p^{2}}$, the threefold $Y_{1}$ is endowed with the $\mathbb{F}_{p^{2}}$

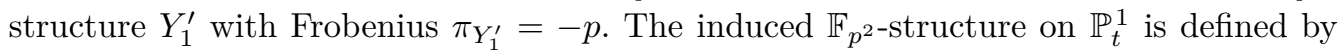

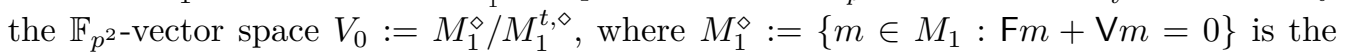
skeleton of $M_{1}$, cf. [13, Section 5.7].

Since $\operatorname{ker}\left(\lambda_{1}\right) \simeq \alpha_{p} \times \alpha_{p}$, the quasi-polarised superspecial Dieudonné module $\left(M_{1},\langle\rangle,\right)$ decomposes into a product of a two-dimensional indecomposable superspecial Dieudonné 
module and a one-dimensional such module. By [13, Proposition 6.1], there is a $W$-basis $e_{1}, e_{2}, e_{3}, f_{1}, f_{2}, f_{3}$ for $M_{1}$ such that $\mathrm{F} e_{i}=-\mathrm{V} e_{i}=f_{i}, \mathrm{~F} f_{i}=-\mathrm{V} f_{i}=-p e_{i}$ for $i=1,2,3$,

$$
\left\langle e_{1}, e_{2}\right\rangle=p^{-1}, \quad\left\langle f_{1}, f_{2}\right\rangle=1, \quad\left\langle e_{3}, f_{3}\right\rangle=1
$$

and other pairings are zero. Then $M_{1}^{\vee}$ is spanned by $p e_{1}, p_{2}, e_{3}, f_{1}, f_{2}, f_{3}$ and $M_{1} / M_{1}^{\vee}=$ $\operatorname{Span}_{k}\left\{e_{1}, e_{2}\right\}$. Let $u=\left(u_{1}: u_{2}\right) \in \mathbb{P}_{t}^{1}(k)$ be the projective coordinates of the point corresponding to $M / M_{1}^{\vee}$. That is, $M / M_{1}^{\vee}$ is the one-dimensional subspace spanned by $u=u_{1} \bar{e}_{1}+u_{2} \bar{e}_{2}$, where $\bar{e}_{i}$ denotes the image of $e_{i}$ in $M_{1} / M_{1}^{\vee}$.

If $u \in \mathbb{P}_{t}^{1}\left(\mathbb{F}_{p^{2}}\right)$, then $a(M)=3$ and $\operatorname{Mass}\left(\Lambda_{x}\right)$ is already computed in Corollary 2.5. Suppose then that $u \notin \mathbb{P}_{t}^{1}\left(\mathbb{F}_{p^{2}}\right)$. In this case, $M_{1}$ (resp. $M_{1}^{\vee}$ ) is the smallest (resp. maximal) superspecial Dieudonné module containing (resp. contained in) $M$. Thus,

$$
\operatorname{End}(M)=\left\{g \in \operatorname{End}\left(M_{1}\right): g\left(M_{1}^{\vee}\right) \subseteq M_{1}^{\vee}, g(M) \subseteq M\right\}
$$

Consider the reduction map

$$
m: \operatorname{End}\left(M_{1}\right)=\operatorname{End}\left(M_{1}^{\diamond}\right) \rightarrow \operatorname{End}\left(M_{1}^{\diamond} / M_{1}^{t, \diamond}\right)=\operatorname{End}_{\mathbb{F}_{p^{2}}}\left(V_{0}\right)=\operatorname{Mat}_{2}\left(\mathbb{F}_{p^{2}}\right)
$$

It is clear that $\operatorname{End}(M)$ contains $\operatorname{ker}(m)$ and that $m$ induces a surjective map

$$
m: \operatorname{End}(M) \rightarrow m(\operatorname{End}(M))=\left\{g \in \operatorname{Mat}_{2}\left(\mathbb{F}_{p^{2}}\right): g \cdot u \subseteq k \cdot u\right\}
$$

Write $\operatorname{End}(u):=\left\{g \in \operatorname{Mat}_{2}\left(\mathbb{F}_{p^{2}}\right): g \cdot u \subseteq k \cdot u\right\}$

\section{Lemma 4.1.}

(1) If $u \in \mathbb{P}_{t}^{1}\left(\mathbb{F}_{p^{4}}\right)-\mathbb{P}_{t}^{1}\left(\mathbb{F}_{p^{2}}\right)$, then $\operatorname{End}(u) \subseteq \operatorname{Mat}_{2}\left(\mathbb{F}_{p^{2}}\right)$ is an $\mathbb{F}_{p^{2}}$-subalgebra which is isomorphic to $\mathbb{F}_{p^{4}}$.

(2) If $u \in \mathbb{P}_{t}^{1}(k)-\mathbb{P}_{t}^{1}\left(\mathbb{F}_{p^{4}}\right)$, then $\operatorname{End}(u)=\mathbb{F}_{p^{2}}$.

Proof. This is a simpler version of Lemmas 3.11 and 3.12 so we omit the proof; cf. also [27, Section 3].

Put $\langle,\rangle_{1}:=p\langle$,$\rangle . Then \langle,\rangle_{1}$ induces a non-degenerate alternating pairing, again denoted $\langle,\rangle_{1}: V_{0} \times V_{0} \rightarrow \mathbb{F}_{p^{2}}$. The reduction map $m$ then gives rise to the following map

$$
m: \operatorname{Aut}\left(M_{1},\langle,\rangle\right)=\operatorname{Aut}\left(M_{1},\langle,\rangle_{1}\right) \rightarrow \operatorname{Aut}\left(V_{0},\langle,\rangle_{1}\right) \simeq \mathrm{SL}_{2}\left(\mathbb{F}_{p^{2}}\right)
$$

Lemma 4.2. The map $m: \operatorname{Aut}\left(M_{1},\langle\rangle,\right) \rightarrow \operatorname{Aut}\left(V_{0},\langle,\rangle_{1}\right)$ is surjective.

Proof. Since $Y_{1}$ is supersingular, we have that $\operatorname{End}\left(Y_{1}\right) \otimes \mathbb{Z}_{p} \simeq \operatorname{End}\left(M_{1}\right)$ and that $G_{x_{1}}\left(\mathbb{Z}_{p}\right) \simeq \operatorname{Aut}\left(M_{1},\langle\rangle,\right)$; recall the notation from (6). The group scheme $G_{x_{1}} \otimes \mathbb{Z}_{p}$ is a 
parahoric group scheme and in particular is smooth over $\mathbb{Z}_{p}$. Thus, the map $G_{x_{1}}\left(\mathbb{Z}_{p}\right) \rightarrow$ $G_{x_{1}}\left(\mathbb{F}_{p}\right)$ is surjective. Now $\operatorname{Aut}\left(V_{0},\langle,\rangle_{1}\right)=\operatorname{Res}_{\mathbb{F}_{p^{2}} / \mathbb{F}_{p}} \mathrm{SL}_{2}$ viewed as an algebraic group over $\mathbb{F}_{p}$ is a reductive quotient of the special fibre $G_{x_{1}} \otimes \mathbb{F}_{p}$. Therefore, the map $G_{x_{1}}\left(\mathbb{F}_{p}\right) \rightarrow$ $\operatorname{Aut}\left(V_{0},\langle,\rangle_{1}\right)=\mathrm{SL}_{2}\left(\mathbb{F}_{p^{2}}\right)$ is also surjective. This proves the lemma.

We now prove the main result of this section.

Theorem 4.3. Let $x=(X, \lambda) \in \mathscr{S}_{3,1}(k)$ with $a(X) \geq 2$ and let $y \in \mathscr{P}_{\mu}^{\prime}(k)$ be a lift of $x$ for some $\mu \in P\left(E^{3}\right)$. Write $y=(t, u)$ where $t=\pi(y) \in C\left(\mathbb{F}_{p^{2}}\right)$ and $u \in \pi^{-1}(t)=\mathbb{P}_{t}^{1}(k)$. Then

$$
\operatorname{Mass}\left(\Lambda_{x}\right)=\frac{L_{p}}{2^{10} \cdot 3^{4} \cdot 5 \cdot 7}
$$

where

$$
L_{p}= \begin{cases}(p-1)\left(p^{2}+1\right)\left(p^{3}-1\right) & \text { if } u \in \mathbb{P}_{t}^{1}\left(\mathbb{F}_{p^{2}}\right) \\ (p-1)\left(p^{3}+1\right)\left(p^{3}-1\right)\left(p^{4}-p^{2}\right) & \text { if } u \in \mathbb{P}_{t}^{1}\left(\mathbb{F}_{p^{4}}\right) \backslash \mathbb{P}_{t}^{1}\left(\mathbb{F}_{p^{2}}\right) \\ 2^{-e(p)}(p-1)\left(p^{3}+1\right)\left(p^{3}-1\right) p^{2}\left(p^{4}-1\right) & \text { if } u \notin \mathbb{P}_{t}^{1}\left(\mathbb{F}_{p^{4}}\right)\end{cases}
$$

where $e(p)=0$ if $p=2$ and $e(p)=1$ if $p>2$.

Proof. By Lemma 4.2,

$$
\left[\operatorname{Aut}\left(M_{1},\langle,\rangle\right): \operatorname{Aut}(M,\langle,\rangle)\right]=\left[\mathrm{SL}_{2}\left(\mathbb{F}_{p^{2}}\right): \mathrm{SL}_{2}\left(\mathbb{F}_{p^{2}}\right) \cap \operatorname{End}(u)^{\times}\right]
$$

By Lemma 4.1,

$$
\mathrm{SL}_{2}\left(\mathbb{F}_{p^{2}}\right) \cap \operatorname{End}(u)^{\times}= \begin{cases}\mathbb{F}_{p^{4}}^{1} & \text { if } u \in \mathbb{P}_{t}^{1}\left(\mathbb{F}_{p^{4}}\right) \backslash \mathbb{P}_{t}^{1}\left(\mathbb{F}_{p^{2}}\right) \\ \{ \pm 1\} & \text { if } u \notin \mathbb{P}_{t}^{1}\left(\mathbb{F}_{p^{4}}\right)\end{cases}
$$

It follows that

$$
\left[\operatorname{Aut}\left(M_{1},\langle\rangle\right): \operatorname{Aut}(M,\langle,\rangle)\right]= \begin{cases}p^{2}\left(p^{2}-1\right) & \text { if } u \in \mathbb{P}_{t}^{1}\left(\mathbb{F}_{p^{4}}\right) \backslash \mathbb{P}_{t}^{1}\left(\mathbb{F}_{p^{2}}\right) \\ \left|\operatorname{PSL}_{2}\left(\mathbb{F}_{p^{2}}\right)\right| & \text { if } u \notin \mathbb{P}_{t}^{1}\left(\mathbb{F}_{p^{4}}\right)\end{cases}
$$

so the theorem follows from (19).

\section{The case $a(X)=1$}

Suppose that $(X, \lambda)$ is a supersingular principally polarised abelian threefold over $k$ with $a(X)=1$. By Proposition 3.16(1), there is a minimal isogeny $\varphi:\left(Y_{2}, \mu\right) \rightarrow(X, \lambda)$, where $Y_{2}=E_{k}^{3}$, and where $\varphi^{*} \lambda=p \mu$ for $\mu \in P\left(E^{3}\right)$ a principal polarisation. In this section we will compute the local index 


$$
\left[\operatorname{Aut}\left(\left(Y_{2}, \mu\right)\left[p^{\infty}\right]\right): \operatorname{Aut}\left((X, \lambda)\left[p^{\infty}\right]\right)\right] .
$$

Let $M$ and $M_{2}$ be the Dieudonné modules of $X$ and $Y_{2}$, respectively. Together with the induced (quasi-)polarisations, we have $(M,\langle\rangle$,$) and \left(M_{2},\langle,\rangle_{2}\right)$, where $\langle,\rangle_{2}=p\langle$,$\rangle is again$ a principal polarisation. (Note that $\left(M_{2},\langle,\rangle_{2}\right)$ is the quasi-polarised Dieudonné module associated to $\left(Y_{2}, \mu\right)$ and not to $\left(Y_{2}, p \mu\right)$, and that $p M_{2} \subseteq M$ by the proof of Proposition 3.16(1).) The proof of Proposition 3.16(1) also shows that every automorphism of $M$ can be lifted to an automorphism of $M_{2}$, i.e., that $\operatorname{Aut}((M,\langle\rangle),) \subseteq \operatorname{Aut}\left(\left(M_{2},\langle,\rangle_{2}\right)\right)$. Then equivalently to (23), cf. Proposition 2.12, we will compute

$$
\left[\operatorname{Aut}\left(\left(M_{2},\langle,\rangle_{2}\right)\right): \operatorname{Aut}((M,\langle,\rangle))\right] .
$$

\subsection{Determining Aut $\left(\left(M_{2},\langle,\rangle_{2}\right)\right)$}

Let $W=W(k)$ denote the ring of Witt vectors over $k$. Choose a $W$-basis $e_{1}, e_{2}, e_{3}, f_{1}, f_{2}, f_{3}$ for $M_{2}$ such that

$$
\mathrm{F} e_{i}=-\mathrm{V} e_{i}=f_{i}, \quad \mathrm{~F} f_{i}=-\mathrm{V} f_{i}=-p e_{i}, \quad\left\langle e_{i}, f_{j}\right\rangle_{2}=\delta_{i j}, \quad\left\langle e_{i}, e_{j}\right\rangle_{2}=\left\langle f_{i}, f_{j}\right\rangle_{2}=0,
$$

for all $i, j \in\{1,2,3\}$.

Let $D_{p}$ be the division quaternion algebra over $\mathbb{Q}_{p}$ and let $\mathscr{O}_{D_{p}}$ denote its maximal order. We also write $D_{p}=\mathbb{Q}_{p^{2}}[\Pi]$ and $\mathscr{O}_{D_{p}}=\mathbb{Z}_{p^{2}}[\Pi]$, where $\mathbb{Z}_{p^{2}}=W\left(\mathbb{F}_{p^{2}}\right)$ and $\mathbb{Q}_{p^{2}}=$ Frac $W\left(\mathbb{F}_{p^{2}}\right)$, and where $\Pi^{2}=-p$ and $\Pi a=\bar{a} \Pi$ for any $a \in \mathbb{Q}_{p^{2}}$. Here $a \mapsto \bar{a}$ denotes the non-trivial automorphism of $\mathbb{Q}_{p^{2}} / \mathbb{Q}_{p}$. If we let $*$ denote the canonical involution of $D_{p}$, then $a^{*}=\bar{a}$ for any $a \in \mathbb{Q}_{p^{2}}$, and $\Pi^{*}=-\Pi$.

Lemma 5.1. We have $\operatorname{End}\left(M_{2}\right) \simeq \operatorname{Mat}_{3}\left(\mathscr{O}_{D_{p}}\right)$ and hence $\operatorname{Aut}\left(M_{2}\right) \simeq \operatorname{GL}_{3}\left(\mathscr{O}_{D_{p}}\right)$ (not taking the polarisation into account).

Proof. We have $\operatorname{End}\left(M_{2}\right)=\operatorname{End}_{\mathscr{O}_{D_{p}}}\left(M_{2}^{\diamond}\right)$, where $M_{2}^{\diamond}:=\left\{m \in M_{2}: \mathrm{F} m+\mathrm{V} m=0\right\}$ denotes the skeleton of $M_{2}$; this is an $\mathscr{O}_{D_{p}}$-module where $\Pi$ acts by $\mathrm{F}$ and $\Pi^{*}$ acts by $\mathrm{V}$. Now the result follows by using the basis $e_{1}, e_{2}, e_{3}$ for $\operatorname{Mat}_{3}\left(\mathscr{O}_{D_{p}}\right)^{\text {op }}$ (the opposite algebra); we choose a convention where the matrices act on the left. We fix the isomorphism $\operatorname{Mat}_{3}\left(\mathscr{O}_{D_{p}}\right)^{\text {op }} \simeq \operatorname{Mat}_{3}\left(\mathscr{O}_{D}\right)$ by sending $A$ to $A^{*}$.

We fix the identification $\operatorname{End}\left(M_{2}\right)=\operatorname{Mat}_{3}\left(\mathscr{O}_{D}\right)$ by the isomorphism chosen in Lemma 5.1 with respect to the basis in (25).

Lemma 5.2. We have $\operatorname{Aut}\left(M_{2},\langle,\rangle_{2}\right) \simeq\left\{A \in \mathrm{GL}_{3}\left(\mathscr{O}_{D_{p}}\right): A^{*} A \simeq \mathbb{I}_{3}\right\}$.

Proof. It suffices to check that $\left\langle A \cdot e_{i}, e_{j}\right\rangle_{2}=\left\langle e_{i}, A^{*} \cdot e_{j}\right\rangle_{2}$ for any $A \in \operatorname{Mat}_{3}\left(\mathscr{O}_{D_{p}}\right)$ and any $i, j \in\{1,2,3\}$. Write $A=\left(a_{i j}\right)$ and $A^{*}=\left(a_{i j}^{\prime}\right)$ with $a_{i j}=c_{i j}+d_{i j} \Pi$ for $c_{i j}, d_{i j} \in \mathbb{Z}_{p^{2}}$, and with $a_{i j}^{\prime}=a_{j i}^{*}$. Then 


$$
\left\langle A \cdot e_{i}, e_{j}\right\rangle_{2}=\left\langle\sum_{k} a_{i k} e_{k}, e_{j}\right\rangle_{2}=\left\langle d_{i j} f_{j}, e_{j}\right\rangle_{2}=-d_{i j}
$$

coincides with

$$
\left\langle e_{i}, A^{*} \cdot e_{j}\right\rangle_{2}=\left\langle e_{i}, \sum_{k} a_{j k}^{\prime} e_{k}\right\rangle_{2}=\left\langle e_{i}, a_{j i}^{\prime} e_{i}\right\rangle_{2}=\left\langle e_{i}, \bar{c}_{i j} e_{i}-d_{i j} f_{i}\right\rangle_{2}=-d_{i j},
$$

as required.

\subsection{Endomorphisms and automorphisms modulo $\mathrm{pM}_{2}$}

As was pointed out earlier, the proof of Proposition 3.16(1) contains the important observation that $p M_{2} \subseteq M$. This allows us to consider the endomorphisms and automorphisms of both $M_{2}$ and $M$ modulo $p$ (i.e., reducing modulo $p M_{2}$ ) and modulo $\Pi$. In Definitions 5.3 and 5.4, we first define, and introduce notation for, all the endomorphism rings and automorphism groups we are considering.

Definition 5.3. Let $m_{p}$ denote the reduction-modulo- $p$ map and $m_{\Pi}$ the reductionmodulo-П map. By Lemma 5.1, for $M_{2}$ we have

$$
\operatorname{End}\left(M_{2}\right) \simeq \operatorname{Mat}_{3}\left(\mathscr{O}_{D_{p}}\right) \stackrel{m_{p}}{\longrightarrow} \operatorname{Mat}_{3}\left(\mathbb{F}_{p^{2}}[\Pi]\right) \stackrel{m_{\Pi}}{\longrightarrow} \operatorname{Mat}_{3}\left(\mathbb{F}_{p^{2}}\right) .
$$

On the level of automorphisms (respecting the polarisation) we get

$$
\operatorname{Aut}\left(M_{2},\langle,\rangle_{2}\right) \stackrel{m_{p}}{\longrightarrow} G_{\left(M_{2},\langle,\rangle_{2}\right)} \stackrel{m_{\Pi}}{\longrightarrow} \bar{G}_{\left(M_{2},\langle,\rangle_{2}\right)},
$$

where

$$
G_{\left(M_{2},\langle,\rangle_{2}\right)}:=\left\{A+B \Pi \in \mathrm{GL}_{3}\left(\mathbb{F}_{p^{2}}[\Pi]\right): A \bar{A}^{T}=\mathbb{I}_{3}, B^{T} \bar{A}=\bar{A}^{T} B\right\}
$$

(here, $B^{T}$ denotes the transpose of the matrix $B$ ), and where

$$
\bar{G}_{\left(M_{2},\langle,\rangle_{2}\right)}:=\left\{A \in \mathrm{GL}_{3}\left(\mathbb{F}_{p^{2}}\right): A^{*} A=\mathbb{I}_{3}\right\}
$$

Definition 5.4. For $M$ we have $\operatorname{End}(M)=\left\{g \in \operatorname{End}\left(M_{2}\right): g(M) \subseteq M\right\}$ and $\operatorname{Aut}(M)=$ $\left\{g \in \operatorname{Aut}\left(M_{2}\right): g(M)=M\right\}$, and

$$
\operatorname{Aut}(M,\langle,\rangle)=\left\{g \in \operatorname{Aut}\left(M_{2},\langle,\rangle_{2}\right): g(M)=M\right\}
$$

Under the same maps $m_{p}$ and $m_{\Pi}$, we find

$$
E_{M}:=m_{p}(\operatorname{End}(M))=\left\{A \in \operatorname{Mat}_{3}\left(\mathbb{F}_{p^{2}}[\Pi]\right): A \cdot M / p M_{2} \subseteq M / p M_{2}\right\}
$$

and $\bar{E}_{M}:=m_{\Pi}\left(E_{M}\right) \subseteq \operatorname{Mat}_{3}\left(\mathbb{F}_{p^{2}}\right)$. These fit in the diagram 


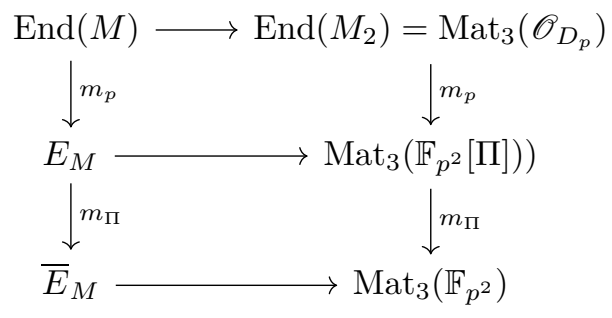

in which all horizontal maps are inclusion maps and the left vertical maps are the surjective reduction maps.

On the level of automorphisms, we let

$$
G_{M}:=m_{p}(\operatorname{Aut}(M))=\left\{A \in \mathrm{GL}_{3}\left(\mathbb{F}_{p^{2}}[\Pi]\right): A \cdot M / p M_{2} \subseteq M / p M_{2}\right\}
$$

and $\bar{G}_{M}:=m_{\Pi}\left(G_{M}\right)$. For the polarised versions, since $\varphi^{*} \lambda=p \mu$, we obtain

$$
G_{(M,\langle,\rangle)}:=\left\{g \in G_{\left(M_{2},\langle,\rangle_{2}\right)}: g\left(M / p M_{2}\right) \subseteq M / p M_{2}\right\}
$$

and

$$
\bar{G}_{(M,\langle,\rangle)}:=\left\{g \in \bar{G}_{\left(M_{2},\langle,\rangle_{2}\right)}: g\left(M / p M_{2}\right) \subseteq M / p M_{2}\right\} .
$$

Denote the group of three-by-three symmetric matrices over $\mathbb{F}_{p^{2}}$ by $S_{3}\left(\mathbb{F}_{p^{2}}\right)$; this group has cardinality $p^{12}$ (since it a six-dimensional $\mathbb{F}_{p^{2} \text {-vector space). Also recall that }}$ the group $U_{3}\left(\mathbb{F}_{p}\right)$ of three-by-three unitary matrices with entries in $\mathbb{F}_{p^{2}}$ has cardinality $p^{3}(p+1)\left(p^{2}-1\right)\left(p^{3}+1\right)$.

Lemma 5.5. In Equation (28) we have $A \in U_{3}\left(\mathbb{F}_{p}\right)$ and $B^{T} \bar{A} \in S_{3}\left(\mathbb{F}_{p^{2}}\right)$. Hence,

$$
\left|G_{\left(M_{2},\langle,\rangle_{2}\right)}\right|=\left|U_{3}\left(\mathbb{F}_{p}\right)\right| \cdot\left|S_{3}\left(\mathbb{F}_{p^{2}}\right)\right|=p^{15}(p+1)\left(p^{2}-1\right)\left(p^{3}+1\right) .
$$

Remark 5.6. Now we note, cf. (24), that

$$
\left[\operatorname{Aut}\left(\left(M_{2},\langle,\rangle_{2}\right)\right): \operatorname{Aut}((M,\langle,\rangle))\right]=\left[G_{\left(M_{2},\langle,\rangle_{2}\right)}: G_{(M,\langle,\rangle)}\right] .
$$

In light of Lemma 5.5, it now suffices to compute $\left[G_{\left(M_{2},\langle,\rangle_{2}\right)}: G_{(M,\langle,\rangle)}\right]$. This will take up the remainder of this section.

We start by studying the unpolarised automorphisms $G_{M_{2}}$. Thus, let $g=\left(a_{i j}+\right.$ $\left.b_{i j} \Pi\right)_{1 \leq i, j \leq 3} \in \mathrm{GL}_{3}\left(\mathbb{F}_{p^{2}}(\Pi)\right)$ be an (unpolarised) automorphism of $M_{2} / p M_{2}$. If we take $\bar{e}_{1}, \bar{e}_{2}, \bar{e}_{3}, \bar{f}_{1}, \bar{f}_{2}, \bar{f}_{3}$ (i.e., the reductions of $e_{1}, \ldots, f_{3}$ in the previous subsection) as a basis of $M_{2} / p M_{2}$ in this order, $g$ can be expressed by a matrix of the form

$$
g=\left(\begin{array}{cc}
A & 0 \\
B & A^{(p)}
\end{array}\right)
$$


where $A=\left(a_{i j}\right)_{1 \leq i, j \leq 3}, B=\left(b_{i j}\right)_{1 \leq i, j \leq 3}$, and $A^{(p)}=\left(a_{i j}^{p}\right)_{1 \leq i, j \leq 3}$.

Recall from Propositions 3.15 and 3.16(1) that the polarised flag type quotient $Y_{2} \rightarrow$ $Y_{1} \rightarrow X$ corresponds to a point $t=\left(t_{1}: t_{2}: t_{3}\right) \in C^{0}(k)$ such that $M_{1} / \mathrm{F} M_{2}$ is generated by $t_{1} \bar{e}_{1}+t_{2} \bar{e}_{2}+t_{3} \bar{e}_{3}$, where $M_{1}$ is the Dieudonné module of $Y_{1}$, and a point $u=\left(u_{1}: u_{2}\right) \in \mathbb{P}_{t}^{1}(k):=\pi^{-1}(t)$. We choose a new basis for $M_{2} / p M_{2}$ as follows:

$$
\begin{aligned}
\bar{E}_{1} & :=\sum_{i=1,2,3} t_{i} \bar{e}_{i}, \bar{E}_{2}:=\sum_{i=1,2,3} t_{i}^{p} \bar{e}_{i}, \bar{E}_{3}:=\sum_{i=1,2,3} t_{i}^{p^{-1}} \bar{e}_{i}, \\
\bar{F}_{1} & :=\sum_{i=1,2,3} t_{i} \bar{f}_{i}, \bar{F}_{2}:=\sum_{i=1,2,3} t_{i}^{p} \bar{f}_{i}, \bar{F}_{3}:=\sum_{i=1,2,3} t_{i}^{p^{-1}} \bar{f}_{i} .
\end{aligned}
$$

(This is a basis by Lemma 3.9.) Using this basis, $g$ is expressed as

$$
g=\left(\begin{array}{cc}
\mathbb{T}^{-1} A \mathbb{T} & 0 \\
\mathbb{T}^{-1} B \mathbb{T} & \mathbb{T}^{-1} A^{(p)} \mathbb{T}
\end{array}\right)
$$

where

$$
\mathbb{T}:=\left(\begin{array}{ccc}
t_{1} & t_{1}^{p} & t_{1}^{p^{-1}} \\
t_{2} & t_{2}^{p} & t_{2}^{p^{-1}} \\
t_{3} & t_{3}^{p} & t_{3}^{p^{-1}}
\end{array}\right)
$$

Now we determine the group $G_{M} \subseteq \mathrm{GL}_{3}\left(\mathbb{F}_{p^{2}}[\Pi]\right)$ of elements preserving $M / p M_{2}$. Any such element will also preserve $M_{1} / p M_{2}$. We prove the following proposition.

Proposition 5.7. Let $g \in \mathrm{GL}_{3}\left(\mathbb{F}_{p^{2}}[\Pi]\right)$ be an automorphism of $M_{2} / p M_{2}$, expressed as in (38). Then $g \in G_{M}$ (i.e., $g$ preserves $M / p M_{2}$ ) if and only if the following hold:

(a) We have $A \cdot t=\alpha t$ for some $\alpha \in k$, i.e., $A \in \operatorname{End}(t)$.

(b) The (1,1)-component of the matrix $\mathbb{T}^{-1} B \mathbb{T}$ is $u_{2} u_{1}^{-1}\left(\alpha-\alpha^{p^{3}}\right)$.

Proof. For an $A \in \operatorname{End}(t)$ (see Definition 3.10) with eigenvalue $\alpha$, it holds by definition that

$$
\mathbb{T}^{-1} A \mathbb{T}=\left(\begin{array}{lll}
\alpha & * & * \\
& * & * \\
& * & *
\end{array}\right), \mathbb{T}^{-1} A^{(p)} \mathbb{T}=\left(\begin{array}{lll}
* & & \\
* & \alpha^{p} & \\
* & & \alpha^{p^{-1}}
\end{array}\right)
$$

As $\operatorname{det}(A)=\alpha^{1+p^{2}+p^{-2}}$ and $\operatorname{det}\left(A^{(p)}\right)=\operatorname{det}(A)^{p}$, we see that

$$
\mathbb{T}^{-1} A^{(p)} \mathbb{T}=\left(\begin{array}{ccc}
\alpha^{p^{3}} & & \\
* & \alpha^{p} & \\
* & & \alpha^{p^{-1}}
\end{array}\right)
$$


By Proposition 3.16(1), the quotient $M_{1} / p M_{2}$ is a two dimensional $k$-vector space generated by $\bar{E}_{1}$ and $\bar{F}_{1}$. As $M_{1}^{\vee}=(\mathrm{F}, \mathrm{V}) M_{1}=p M_{2}$, we find that $M / p M_{2} \subseteq M_{1} / p M_{2}$ is a one-dimensional $k$-vector space. Take $u_{1}, u_{2} \in k$ so that $M / p M_{2}$ is generated by the image of $u_{1} \bar{E}_{1}+u_{2} \bar{F}_{1}$. As $M \neq p M_{2}$, we see that $u_{1} \neq 0$.

We see that if $g \in \mathrm{GL}_{3}\left(\mathbb{F}_{p^{2}}[\Pi]\right)$ preserves $M_{1} /(\mathrm{F}, \mathrm{V}) M_{2}$, then it induces an automorphism of $M_{1} /(\mathrm{F}, \mathrm{V}) M_{1}=M_{1} / p M_{2}$ which is expressed as $\left(\begin{array}{l}\alpha \\ * \alpha^{p^{3}}\end{array}\right)$ by (39), (41), and (42). Moreover, $g$ also preserves $M /(\mathrm{F}, \mathrm{V}) M_{1}=M / p M_{2}$ if and only if the column vector $\left(\begin{array}{ll}\alpha \\ * \alpha^{p^{3}}\end{array}\right)\left(\begin{array}{l}u_{1} \\ u_{2}\end{array}\right)$ is in the subspace spanned by $\left(\begin{array}{l}u_{1} \\ u_{2}\end{array}\right)$. This is equivalent to the entry $*$ being equal to $u_{2} u_{1}^{-1}\left(\alpha-\alpha^{p^{3}}\right)$.

\section{Remark 5.8.}

(1) It follows from the construction of polarised flag type quotients that for $(X, \lambda)$ with $a(X)=1$ and a choice $\mu \in P\left(E^{3}\right)$ together with an identification $(\tilde{X}, \tilde{\lambda})=\left(E_{k}^{3}, p \mu\right)$, there exists a unique pair $(t, u)$ where $t=\left(t_{1}: t_{2}: t_{3}\right) \in C^{0}(k)$ and $u=\left(u_{1}: u_{2}\right) \in$ $\mathbb{P}^{1}(k)$ as in the proof of Proposition 5.7. For the rest of the section, we will work with these $(t, u)$.

(2) The coordinates $(t, u)$ in (1) also give rise to a trivialisation $C^{0} \times \mathbb{P} \simeq \mathscr{P}_{C^{0}}$, where $\mathscr{P}_{C^{0}}:=\mathscr{P}_{\mu} \times_{C} C^{0}$, as follows. By Proposition 3.7, points in $\mathscr{P}_{C^{0}}$ correspond to pairs $\left(\bar{M}_{1}, \bar{M}\right)$ : here $\bar{M}_{1} \subseteq \bar{M}_{2}$ is a four-dimensional subspace generated by the subspace $\vee \bar{M}_{2}$ and $\bar{E}_{1}=t_{1} \bar{e}_{1}+t_{2} \bar{e}_{2}+t_{3} \bar{e}_{3}$ with $\left(t_{1}: t_{2}: t_{3}\right) \in C^{0}$, and $\bar{M} \subseteq \bar{M}_{2}$ is a three-dimensional subspace with $\bar{M}_{1}^{\perp} \subseteq \bar{M} \subseteq \bar{M}_{1}$, where $\bar{M}_{1}^{\perp}$ is the orthogonal complement of $\bar{M}_{1}$ with respect to $\langle,\rangle_{2}$. The two-dimensional vector spaces $\bar{M}_{1} / \bar{M}_{1}^{\perp}$ for $t \in C^{0}$ form a rank two vector bundle $\mathscr{V}=\left.\mathscr{O}(1) \oplus \mathscr{O}(-1)\right|_{C^{0}}$ over $C^{0}$. As shown in the proof of Proposition 5.7, the images of $\bar{E}_{1}$ and $\bar{F}_{1}$ in $\bar{M}_{1} / \bar{M}_{1}^{\perp}$ (again denoted by $\bar{E}_{1}$ and $\bar{F}_{1}$ for simplicity) form a basis, and give rise to two global sections $\widetilde{E}_{1}$ and $\widetilde{F}_{1}$ of $\mathscr{V}$ respectively (note that both $\bar{E}_{1}$ and $\bar{F}_{1}$ are vector-valued functions in $t_{1}, t_{2}$, and $\left.t_{3}\right)$. Then the desired trivialisation $C^{0} \times \mathbb{P} \stackrel{\sim}{\longrightarrow} \mathscr{P}_{C^{0}} \simeq \mathbb{P}(\mathscr{V})$ is given by $\left(t,\left(u_{1}: u_{2}\right)\right) \mapsto\left[u_{1} \widetilde{E}_{1}(t)+u_{2} \widetilde{F}_{1}(t)\right]$. Since $M_{2}$ is the Dieudonné module of $E_{k}^{3}$, the vector space $\bar{M}_{2}$ has an $\mathbb{F}_{p^{2} \text {-structure, so we see that this trivialisation is defined }}$ over $\mathbb{F}_{p^{2}}$.

Now let $t \in C^{0}(k)$ and $u=(0: 1)$. The corresponding subspace $\bar{M}$ is generated by $\bar{F}_{1}$ and $\bar{M}_{1}^{\perp}=(\mathrm{F}, \mathrm{V}) \bar{M}_{1}$. Therefore, we have $\bar{M}=\mathrm{\vee} \bar{M}_{2}$, which corresponds a point in $T$. It follows that under the above trivialisation, $\left.T\right|_{C^{0}} \simeq C^{0} \times\{\infty\}$.

The following lemma follows from Lemma 3.11, Lemma 3.12, and Proposition 5.7. It describes the polarised elements $g \in G_{\left(M_{2},\langle,\rangle_{2}\right)}$ that preserve $M_{1} / p M_{2}$ : for such $g$ of the form (38), Proposition 5.7(1) implies that $A \in \operatorname{End}(t)$, while Definition 5.3(28) implies that $A$ is unitary.

Lemma 5.9. Let $t=\left(t_{1}: t_{2}: t_{3}\right) \in C^{0}(k)$. 
(1) When $t \notin C\left(\mathbb{F}_{p^{6}}\right)$, we have

$$
\operatorname{End}(t) \cap U_{3}\left(\mathbb{F}_{p}\right) \simeq\left\{\alpha \in \mathbb{F}_{p^{2}}: \alpha^{p+1}=1\right\}
$$

(2) When $t \in C\left(\mathbb{F}_{p^{6}}\right)$, we have

$$
\operatorname{End}(t) \cap U_{3}\left(\mathbb{F}_{p}\right) \simeq\left\{\alpha \in \mathbb{F}_{p^{6}}: \alpha^{p^{3}+1}=1\right\}
$$

Proof. (1) This follows since a diagonal matrix $\alpha \mathbb{I}_{3}$ with $\alpha \in \mathbb{F}_{p^{2}}$ is unitary if and only if $\alpha^{p+1}=1$.

(2) Take any $A \in \operatorname{End}(t) \cap U_{3}\left(\mathbb{F}_{p}\right)$. The eigenvalues of $A^{(p) T}$ are $\alpha^{p}, \alpha^{p^{3}}, \alpha^{p^{5}}$ where $\alpha$ is the eigenvalue of $A$. As $A$ is unitary, $\alpha^{-1}$ is also an eigenvalue, so we have $\alpha^{-1} \in\left\{\alpha^{p}, \alpha^{p^{3}}, \alpha^{p^{5}}\right\}$. In each case, we have $\alpha^{p^{3}+1}=1$.

For the converse, choose any $\alpha \in \mathbb{F}_{p^{6}}$ such that $\alpha^{p^{3}+1}=1$. By the proof of Lemma 3.11, the corresponding $A \in \operatorname{End}(t)$ is given by

$$
A=\left(t, t^{\left(p^{2}\right)}, t^{\left(p^{4}\right)}\right) \operatorname{diag}\left(\alpha, \alpha^{p^{2}}, \alpha^{p^{4}}\right)\left(t, t^{\left(p^{2}\right)}, t^{\left(p^{4}\right)}\right)^{-1} .
$$

We compute that

$$
A A^{(p) T}=\left(t, t^{\left(p^{2}\right)}, t^{\left(p^{4}\right)}\right)\left(\begin{array}{lll} 
& s^{-1} & \\
& & s^{-p^{2}} \\
s^{-p} & &
\end{array}\right)\left(t^{(p)}, t^{\left(p^{3}\right)}, t^{\left(p^{5}\right)}\right)^{T}
$$

where $s=t_{1}^{p^{3}+1}+t_{2}^{p^{3}+1}+t_{3}^{p^{3}+1}$. That is, $A A^{(p) T}$ is independent of $\alpha$. By the case $\alpha=1$, we have $A A^{(p) T}=1$.

Suppose now that we have $g \in G_{\left(M_{2},\langle,\rangle_{2}\right)}$ of the form (38) preserving $M_{1} / p M_{2}$, i.e., we have $A \in \operatorname{End}(t) \cap U_{3}\left(\mathbb{F}_{p}\right)$ by Lemma 5.9. We now determine the conditions on $B$ so that $g$ also preserves $M / p M_{2}$, i.e., so that $g \in G_{(M,\langle,\rangle)}$. By (28), $B$ satisfies a symmetric condition.

Let $S_{3}\left(\mathbb{F}_{p^{2}}\right) A$ (for $A \in \operatorname{End}(t) \cap U_{3}\left(\mathbb{F}_{p}\right)$ as above) be the $\mathbb{F}_{p^{2}}$-vector space consisting of matrices of the form $S A$ for some $S \in S_{3}\left(\mathbb{F}_{p^{2}}\right)$. Define a homomorphism of $\mathbb{F}_{p^{2} \text {-vector }}$ spaces

$$
\begin{aligned}
\psi_{t, A}: S_{3}\left(\mathbb{F}_{p^{2}}\right) A \rightarrow k \\
S A \mapsto \text { the }(1,1) \text {-component of } \mathbb{T}^{-1} S A \mathbb{T} .
\end{aligned}
$$

Similarly define a homomorphism

$$
\begin{aligned}
\psi_{t}: S_{3}\left(\mathbb{F}_{p^{2}}\right) & \rightarrow k \\
S & \mapsto \text { the }(1,1) \text {-component of } \mathbb{T}^{-1} S \mathbb{T}
\end{aligned}
$$

Using these notations, we have the following proposition. 
Proposition 5.10. The group $G_{(M,\langle,\rangle)}$ consists of the matrices of the form

$$
\left(\begin{array}{cc}
A & 0 \\
S A & A^{(p)}
\end{array}\right)
$$

satisfying the following conditions:

(1) $A \in \operatorname{End}(t) \cap U_{3}\left(\mathbb{F}_{p}\right)$ with eigenvalue $\alpha$;

(2) $S \in S_{3}\left(\mathbb{F}_{p^{2}}\right)$ is a symmetric matrix; and

(3) $\psi_{t, A}(S A)=u_{2} u_{1}^{-1}\left(\alpha-\alpha^{p^{3}}\right)$.

The third condition is equivalent to

$\left(3^{\prime}\right) \psi_{t}(S)=u_{2} u_{1}^{-1}\left(1-\alpha^{p^{3}-1}\right)$.

Proof. It follows from (34) and Proposition 5.7 that for $A \in \operatorname{End}(t) \cap U_{3}\left(\mathbb{F}_{p}\right)$ with eigenvalue $\alpha$, the matrix $\left(\begin{array}{cc}A & 0 \\ B & A^{(p)}\end{array}\right)$ is an element of $G_{\left(M,\langle,\rangle_{2}\right)} \cap G_{(M,\langle,\rangle)}$ if and only if $B A^{-1}$ is a symmetric matrix and the $(1,1)$-component of the matrix $\mathbb{T}^{-1} B \mathbb{T}$ is $u_{2} u_{1}^{-1}\left(\alpha-\alpha^{p^{3}}\right)$. The latter condition amounts to Condition (3) (and (3')) by noticing that since $\mathbb{T}^{-1} A \mathbb{T}$ is of the form

$$
\left(\begin{array}{lll}
\alpha & * & * \\
& * & * \\
& * & *
\end{array}\right)
$$

where $\alpha$ is the eigenvalue of $A$, we have a commutative diagram

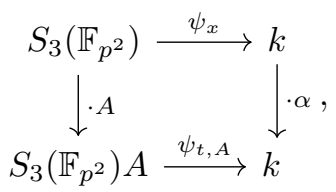

where the left vertical arrow is multiplying $A$ from the right and the right vertical arrow is multiplying with $\alpha$.

The following corollary follows immediately from Proposition 5.10 and summarises the results in this subsection.

Corollary 5.11. We have

$$
\left|G_{(M,\langle,\rangle)}\right|=\left|\left\{A \in \operatorname{End}(t) \cap U_{3}\left(\mathbb{F}_{p}\right): u_{2} u_{1}^{-1}\left(1-\alpha^{p^{3}-1}\right) \in \operatorname{Im}\left(\psi_{t}\right)\right\}\right| \cdot\left|\operatorname{ker}\left(\psi_{t}\right)\right|
$$




\subsection{Analysing $\operatorname{Im}\left(\psi_{t}\right)$ and $\operatorname{ker}\left(\psi_{t}\right)$}

In the following subsection, we will make Corollary 5.11 more explicit by analysing the image and kernel of the homomorphism $\psi_{t}$.

Definition 5.12. In the notation as above, we set

$$
d(t):=\operatorname{dim}_{\mathbb{F}_{p^{2}}}\left(\operatorname{Im}\left(\psi_{t}\right)\right)
$$

As $\operatorname{dim}_{\mathbb{F}_{p^{2}}}\left(S_{3}\left(\mathbb{F}_{p^{2}}\right)\right)=6$, we see that $d(t) \leq 6$, and that

$$
\left|\operatorname{ker}\left(\psi_{t}\right)\right|=p^{2(6-d(t))}
$$

We prove the following precise result about the values of $d(t)$.

Proposition 5.13. We have $3 \leq d(t) \leq 6$. When $p=2$, we have $d(t)=3$. Let $v=$ $\left(t_{1}^{2}, t_{2}^{2}, t_{3}^{2}, t_{1} t_{2}, t_{1} t_{3}, t_{2} t_{3}\right)$ and let

$$
\Delta=\left\{\operatorname{det}\left(v^{T},\left(v^{\left(p^{2}\right)}\right)^{T},\left(v^{\left(p^{4}\right)}\right)^{T}, \ldots,\left(v^{\left(p^{10}\right)}\right)^{T}\right)=0\right\}
$$

When $p \neq 2$, we have:

$$
\begin{array}{lll}
d(t)=3 & \text { if and only if } & t \in C^{0}\left(\mathbb{F}_{p^{6}}\right) ; \\
d(t)=4 & \text { if and only if } & t \in C^{0}\left(\mathbb{F}_{p^{8}}\right) ; \\
d(t)=5 & \text { if and only if } & t \in \Delta \cap C^{0} \backslash\left(C^{0}\left(\mathbb{F}_{p^{6}}\right) \amalg C^{0}\left(\mathbb{F}_{p^{8}}\right)\right) ; \\
d(t)=6 & \text { if and only if } & t \notin \Delta \cap C^{0} .
\end{array}
$$

Proof. Since $t \in C^{0}(k)$, we see that $t_{i} \neq 0$, and without loss of generality we assume that $t_{3}=1$. For $1 \leq i, j \leq 3$, let $I_{i j}$ be the three-by-three matrix whose $(i, j)$-component is one and where all other entries are zero. Then $I_{11}, I_{22}, I_{33}, I_{12}+I_{21}, I_{13}+I_{31}, I_{23}+I_{32}$ is a basis for $S_{3}\left(\mathbb{F}_{p^{2}}\right)$ over $\mathbb{F}_{p^{2}}$. We set

$$
\begin{aligned}
& w_{1}=\psi_{t}\left(I_{11}\right), w_{2}=\psi_{t}\left(I_{22}\right), w_{3}=\psi_{t}\left(I_{33}\right), \\
& w_{4}=\psi_{t}\left(I_{12}+I_{21}\right), w_{5}=\psi_{t}\left(I_{13}+I_{31}\right), w_{6}=\psi_{t}\left(I_{23}+I_{32}\right) .
\end{aligned}
$$

Lemma 5.14. The $w_{i}$ in (50) satisfy the following relations:

$$
\begin{aligned}
& w_{1}=t_{1}^{2} w_{3}, \quad w_{2}=t_{2}^{2} w_{3}, \\
& w_{4}=2 t_{1} t_{2} w_{3}, \\
& w_{5}=2 t_{1} w_{3}, \quad w_{6}=2 t_{2} w_{3},
\end{aligned}
$$

and $w_{3}$ is not zero. 
Proof. The inverse matrix of $\mathbb{T}$ is

$$
\mathbb{T}^{-1}=\operatorname{det}(\mathbb{T})^{-1}\left(\begin{array}{ccc}
t_{2}^{p}-t_{2}^{p^{-1}} & t_{1}^{p^{-1}}-t_{1}^{p} & t_{1}^{p_{1}^{p^{-1}}}-t_{1}^{p^{-1}} t_{2}^{p} \\
t_{2}^{p^{-1}}-t_{2} & t_{1}-t_{1}^{p^{-1}} & t_{1}^{p^{-1}} t_{2}-t_{1} t_{2}^{p^{-1}} \\
t_{2}^{p}-t_{2} & t_{1}-t_{1}^{p} & t_{1}^{p} t_{2}-t_{1} t_{2}^{p}
\end{array}\right) .
$$

Since for any matrices $M=\left(m_{i j}\right), N=\left(n_{i j}\right)$ and $L=\left(l_{i j}\right)$ the $(1,1)$-component of $M N L$ is given by $\sum_{i, j} m_{1 i} n_{i j} l_{j 1}$, we have

$$
\begin{aligned}
& w_{1}=\operatorname{det}(\mathbb{T})^{-1}\left(t_{2}^{p}-t_{2}^{p^{-1}}\right) t_{1} \\
& w_{2}=\operatorname{det}(\mathbb{T})^{-1}\left(t_{1}^{p^{-1}}-t_{1}^{p}\right) t_{2} .
\end{aligned}
$$

Furthermore, $w_{3}$ is given by

$$
\begin{aligned}
w_{3} & =\operatorname{det}(\mathbb{T})^{-1}\left(t_{1}^{p} t_{2}^{p^{-1}}-t_{1}^{p^{-1}} t_{2}^{p}\right) \\
& =\operatorname{det}(\mathbb{T})^{-1} t_{1}^{-1}\left(t_{1}^{p+1} t_{2}^{p^{-1}}-t_{1}^{p^{-1}+1} t_{2}^{p}\right) \\
& =\operatorname{det}(\mathbb{T})^{-1} t_{1}^{-1}\left(t_{2}^{p}-t_{2}^{p^{-1}}\right) .
\end{aligned}
$$

For the last equality, we used equations $t_{1}^{p+1}+t_{2}^{p+1}+1=0$ and $t_{1}^{p^{-1}+1}+t_{2}^{p^{-1}+1}+1=0$. Similarly, we see that $w_{3}=\operatorname{det}(\mathbb{T})^{-1} t_{2}^{-1}\left(t_{1}^{p^{-1}}-t_{1}^{p}\right)$. These computations imply the first two relations of the assertion, and since $t_{1}, t_{2} \notin \mathbb{F}_{p^{2}}$, we see that $w_{3}$ is not zero. Furthermore, we compute that

$$
\begin{aligned}
w_{4} & =\operatorname{det}(\mathbb{T})^{-1}\left(\left(t_{2}^{p}-t_{2}^{p^{-1}}\right) t_{2}+\left(t_{1}^{p^{-1}}-t_{1}^{p}\right) t_{1}\right) \\
& =\operatorname{det}(\mathbb{T})^{-1}\left(t_{2}^{p+1}-t_{2}^{p^{-1}+1}+t_{1}^{p^{-1}+1}-t_{1}^{p+1}\right) \\
& =2 \operatorname{det}(\mathbb{T})^{-1} t_{2}\left(t_{2}^{p}-t_{2}^{p^{-1}}\right) ; \\
w_{5} & =\operatorname{det}(\mathbb{T})^{-1}\left(\left(t_{2}^{p}-t_{2}^{p^{-1}}\right)+\left(t_{1}^{p_{2}^{p^{-1}}}-t_{1}^{p^{-1}} t_{2}^{p}\right) t_{1}\right) \\
& =\operatorname{det}(\mathbb{T})^{-1}\left(t_{2}^{p}-t_{2}^{p^{-1}}+t_{1}^{p+1} t_{2}^{p^{-1}}-t_{1}^{p^{-1}+1} t_{2}^{p}\right) \\
& =2 \operatorname{det}(\mathbb{T})^{-1}\left(t_{2}^{p}-t_{2}^{p^{-1}}\right) .
\end{aligned}
$$

Similarly, we see that $w_{6}=2 \operatorname{det}(\mathbb{T})^{-1}\left(t_{1}^{p^{-1}}-t_{1}^{p}\right)$, so we obtain the remaining relations.

When $p \neq 2$, we see from Lemma 5.14 that

$$
d(t)=\operatorname{dim}_{\mathbb{F}_{p^{2}}}\left\langle w_{1}, w_{2}, w_{3}, w_{4}, w_{5}, w_{6}\right\rangle=\operatorname{dim}_{\mathbb{F}_{p^{2}}}\left\langle 1, t_{1}, t_{2}, t_{1} t_{2}, t_{1}^{2}, t_{2}^{2}\right\rangle .
$$

In particular, this implies that 


$$
d(t) \geq \operatorname{dim}_{\mathbb{F}_{p^{2}}}\left\langle w_{3}, w_{5}, w_{6}\right\rangle=\operatorname{dim}_{\mathbb{F}_{p^{2}}}\left\langle 1, t_{1}, t_{2}\right\rangle=3
$$

When $p=2$, by Lemma 3.9 and Lemma 5.14, we see that $d(t)=3$. So assume $p \neq 2$, and consider (49).

By construction ( since $t_{3}=1$ ), we have $t \in \Delta$ if and only if $\operatorname{dim}_{\mathbb{F}_{p^{2}}}\left\langle 1, t_{1}, t_{2}, t_{1} t_{2}, t_{1}^{2}, t_{2}^{2}\right\rangle$ $\leq 5$. Hence we see that $t \in \Delta \cap C^{0}$ if and only if $d(t) \leq 5$, which gives the required statement for $d(t)=6$. Also note that if $d(t) \leq 5$ then there exists some conic $Q / \mathbb{F}_{p^{2}}$ with equation $a_{1}+a_{2} t_{1}+a_{3} t_{2}+a_{4} t_{1} t_{2}+a_{5} t_{1}^{2}+a_{6} t_{2}^{2}=0$ such that $t \in C^{0} \cap Q$. Similarly if $d(t) \leq 4$ then there exist two independent conics $Q_{1}, Q_{2}$ such that $t \in C^{0} \cap Q_{1} \cap Q_{2}$. In this case, $Q_{1}$ and $Q_{2}$ do not have a common component (even defined over $\overline{\mathbb{F}}_{p}$ ). Otherwise, the intersection $Q_{1} \cap Q_{2}$ must be a line $L$ defined over $\mathbb{F}_{p^{2}}$ (because we require $Q_{1} \neq Q_{2}$ ) and $Q_{1}=L \cup L_{1}$ for another line $L_{1}$ defined over $\mathbb{F}_{p^{2}}$. This implies that $t \in L$ or $t \in L_{1}$, a contradiction by Lemma 3.9. If $d(t) \leq 3$ there exist three independent conics $Q_{1}, Q_{2}, Q_{3}$ such that $t \in C^{0} \cap Q_{1} \cap Q_{2} \cap Q_{3}$.

If $t \in C^{0}\left(\mathbb{F}_{p^{2 a}}\right)$ then $d(t) \leq a$, i.e., if $2 \leq \operatorname{deg}_{\mathbb{F}_{p^{2}}}(t) \leq a$ then $d(t) \leq a$, for any value of $a$. This shows in particular that if $t \in C^{0}\left(\mathbb{F}_{p^{6}}\right)$, then $d(t)=3$, cf. Lemma 3.9. Conversely, since $\left|Q_{1} \cap Q_{2}\right| \leq 4$ by Bézout's theorem we see that if $d(t) \leq 4$ then $\operatorname{deg}_{\mathbb{F}_{p^{2}}}(t) \leq 4$. That is, then $t \in C^{0}\left(\mathbb{F}_{p^{8}}\right) \cup C^{0}\left(\mathbb{F}_{p^{6}}\right)$; note that by Lemma 3.8 we have $C^{0}\left(\mathbb{F}_{p^{4}}\right)=\emptyset$.

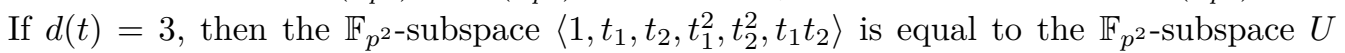
spanned by $1, t_{1}, t_{2}$. Since $t_{1} U \subseteq U$ and $t_{2} U \subseteq U$, the algebra $\mathbb{F}_{p^{2}}\left[t_{1}, t_{2}\right]=U$ has dimension three and $\operatorname{deg}_{\mathbb{F}_{p^{2}}}(t)=3$. This implies that $d(t)=3$ if and only if $t \in C^{0}\left(\mathbb{F}_{p^{6}}\right)$ and hence $d(t)=4$ if and only if $t \in C^{0}\left(\mathbb{F}_{p^{8}}\right)$. The statement for $d(t)=5$ now follows.

Remark 5.15. We provide another proof of the implication $d(t)=3 \Longrightarrow \operatorname{deg}_{\mathbb{F}_{p^{2}}}(t)=3$, since this information may also be useful. Suppose $P_{1}, P_{2}, P_{3}, P_{4} \in \mathbb{P}^{2}(K)$, where $K$ is a field, are four distinct points not on the same line. Then the conics passing through them form a $\mathbb{P}^{1}$-family. To see this, suppose $Q$ is represented by $F(t)=0$, where $F(t)=$ $a_{1} t_{1}^{2}+a_{2} t_{2}^{2}+a_{3} t_{3}^{2}+a_{4} t_{1} t_{2}+a_{5} t_{1} t_{2}+a_{6} t_{1} t_{3}$. By assumption $P_{1}, P_{2}, P_{3}$ are not on the same line. Choose a coordinate for $\mathbb{P}^{2}$ over $K$ such that $P_{1}=(1: 0: 0), P_{2}=(0: 1: 0)$ and $P_{3}=(0: 0: 1)$. Then $a_{1}=a_{2}=a_{3}=0$. The point $P_{4}=\left(\alpha_{1}: \alpha_{2}: \alpha_{3}\right)$ satisfies $\left(\alpha_{1} \alpha_{2}, \alpha_{1} \alpha_{3}, \alpha_{2} \alpha_{3}\right) \neq(0,0,0)$. Thus, $F\left(P_{4}\right)=0$ gives a non-trivial linear relation among $a_{4}, a_{5}$, and $a_{6}$.

Suppose now $t \in C^{0} \cap Q_{1} \cap Q_{2} \cap Q_{3}$ with $\mathbb{F}_{p^{2}}$-linear independent conics $Q_{1}, Q_{2}, Q_{3}$. It suffices to prove $\left|Q_{1} \cap Q_{2} \cap Q_{3}\right| \leq 3$. If $\left|Q_{1} \cap Q_{2}\right| \leq 3$, then we are done. So suppose that $Q_{1} \cap Q_{2}=\left\{P_{1}, P_{2}, P_{3}, P_{4}\right\}$. If $Q_{3}$ contains these four points, then $Q_{3}$ is a linear combination of $Q_{1}$ and $Q_{2}$ over some extension of $\mathbb{F}_{p^{2}}$ and by descent an $\mathbb{F}_{p^{2}}$-linear combination of $Q_{1}$ and $Q_{2}$, contradiction. Thus, we have shown that $\left|Q_{1} \cap Q_{2} \cap Q_{3}\right| \leq 3$.

Definition 5.16. Let $\mathscr{P}_{C^{0}} \simeq C^{0} \times \mathbb{P}^{1}$ be the fibre $\mathbb{P}_{C}(\mathscr{O}(-1) \oplus \mathscr{O}(1)) \times{ }_{C} C^{0}$ over $C^{0}$, cf. Remark 5.8. For each $S \in S_{3}\left(\mathbb{F}_{p^{2}}\right)$, we define a morphism $f_{S}: C^{0} \rightarrow \mathscr{P}_{C^{0}}$ via the map $C^{0} \ni t=\left(t_{1}: t_{2}: t_{3}\right) \mapsto\left(t^{(p)},\left(1: \psi_{t}(S)^{p}\right)\right) \in C^{0} \times \mathbb{P}^{1}$. Observe from the computation in the proof of Proposition 5.13 that $\psi_{t}(S)$ is a polynomial function in $t_{1}^{p^{-1}}, t_{2}^{p^{-1}}, t_{3}^{p^{-1}}$, and 
hence that $\psi_{t}(S)^{p}$ is a polynomial function in $t_{1}, t_{2}, t_{3}$. The image of $f_{S}$ defines a Cartier divisor $\mathscr{D}_{S} \subseteq \mathscr{P}_{C^{0}}$, and we let $\mathscr{D}$ be the horizontal divisor

$$
\mathscr{D}=\sum_{S \in S_{3}\left(\mathbb{F}_{p^{2}}\right)} \mathscr{D}_{S}
$$

For $t \in C^{0}(k)$, let $\mathscr{D}_{t}=\pi^{-1}(t) \cap \mathscr{D}$. That is, $\left(u_{1}: u_{2}\right) \in \mathscr{D}_{t}$ if and only if $u_{2} u_{1}^{-1} \in \operatorname{Im}\left(\psi_{t}\right)$.

Lemma 5.17. Let $t=\left(t_{1}: t_{2}: t_{3}\right) \in C^{0}(k)$.

(1) If $t \notin C^{0}\left(\mathbb{F}_{p^{6}}\right)$, then

$$
\left\{\alpha \in \mathbb{F}_{p^{2}}^{\times}: u_{2} u_{1}^{-1}\left(1-\alpha^{p^{3}-1}\right) \in \operatorname{Im}\left(\psi_{t}\right)\right\}= \begin{cases}\mathbb{F}_{p^{2}}^{\times} & \text {if }\left(u_{1}: u_{2}\right) \in \mathscr{D}_{t} \\ \mathbb{F}_{p}^{\times} & \text {otherwise. }\end{cases}
$$

(2) If $t \in C^{0}\left(\mathbb{F}_{p^{6}}\right)$, then

$$
\left\{\alpha \in \mathbb{F}_{p^{6}}^{\times}: u_{2} u_{1}^{-1}\left(1-\alpha^{p^{3}-1}\right) \in \operatorname{Im}\left(\psi_{t}\right)\right\}= \begin{cases}\mathbb{F}_{p^{6}}^{\times} & \text {if }\left(u_{1}: u_{2}\right) \in \mathscr{D}_{t} \\ \mathbb{F}_{p^{3}}^{\times} & \text {otherwise. }\end{cases}
$$

Proof. (1) First we note that $\mathbb{F}_{p}^{\times} \subseteq\left\{\alpha \in \mathbb{F}_{p^{2}}^{\times}: u_{2} u_{1}^{-1}\left(1-\alpha^{p^{3}-1}\right) \in \operatorname{Im}\left(\psi_{t}\right)\right\}$. Since $\operatorname{Im}\left(\psi_{t}\right)$

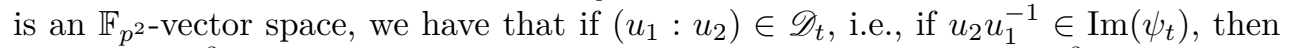
$u_{2} u_{1}^{-1}\left(1-\alpha^{p^{3}-1}\right) \in \operatorname{Im}\left(\psi_{t}\right)$ for any $\alpha \in \mathbb{F}_{p^{2}}^{\times}$. Conversely if $u_{2} u_{1}^{-1}\left(1-\alpha^{p^{3}-1}\right) \in \operatorname{Im}\left(\psi_{t}\right)$ for some $\alpha \in \mathbb{F}_{p^{2}} \backslash \mathbb{F}_{p}$, then $u_{2} u_{1}^{-1} \in \operatorname{Im}\left(\psi_{t}\right)$.

(2) If $t \in C^{0}\left(\mathbb{F}_{p^{6}}\right)$, then $\operatorname{Im}\left(\psi_{t}\right) \subseteq \mathbb{F}_{p^{6}}$. Since $\operatorname{dim}_{\mathbb{F}_{p^{2}}}\left(\mathbb{F}_{p^{6}}\right)=3$ and $d(t) \geq 3$ by Proposition 5.13, we must have that $\operatorname{Im}\left(\psi_{t}\right)=\mathbb{F}_{p^{6}}$. The proof now follows from a similar argument as in (1).

Corollary 5.18. We have

$$
\begin{aligned}
& \left\{A \in \operatorname{End}(t) \cap U_{3}\left(\mathbb{F}_{p}\right): u_{2} u_{1}^{-1}\left(1-\alpha^{p^{3}-1}\right) \in \operatorname{Im}\left(\psi_{t}\right)\right\} \simeq \\
& \begin{cases}\left\{\alpha \in \mathbb{F}_{p}: \alpha^{p+1}=1\right\} & \text { if } t \notin C^{0}\left(\mathbb{F}_{p^{6}}\right) \text { and } u \notin \mathscr{D}_{t} ; \\
\left\{\alpha \in \mathbb{F}_{p^{2}}: \alpha^{p+1}=1\right\} & \text { if } t \notin C^{0}\left(\mathbb{F}_{p^{6}}\right) \text { and } u \in \mathscr{D}_{t} ; \\
\left\{\alpha \in \mathbb{F}_{p^{3}}: \alpha^{p^{3}+1}=1\right\} & \text { if } t \in C^{0}\left(\mathbb{F}_{p^{6}}\right) \text { and } u \notin \mathscr{D}_{t} ; \\
\left\{\alpha \in \mathbb{F}_{p^{6}}: \alpha^{p^{3}+1}=1\right\} & \text { if } t \in C^{0}\left(\mathbb{F}_{p^{6}}\right) \text { and } u \in \mathscr{D}_{t} .\end{cases}
\end{aligned}
$$

Proof. This follows from combining Lemma 5.9 with Lemma 5.17.

\subsection{Determining $\left[\operatorname{Aut}\left(\left(M_{2},\langle,\rangle_{2}\right)\right): \operatorname{Aut}((M,\langle\rangle)),\right]$}

By Corollary 5.11, Equation (48), and the results in the previous subsection, in particular Corollary 5.18, we immediately obtain the following result. 
Lemma 5.19. Define $e(p)=0$ if $p=2$ and $e(p)=1$ if $p>2$. Then

$$
\left|G_{(M,\langle,\rangle)}\right|= \begin{cases}2^{e(p)} p^{2(6-d(t))} & \text { if } u \notin \mathscr{D}_{t} ; \\ (p+1) p^{2(6-d(t))} & \text { if } t \notin C^{0}\left(\mathbb{F}_{p^{6}}\right) \text { and } u \in \mathscr{D}_{t} \\ \left(p^{3}+1\right) p^{6} & \text { if } t \in C^{0}\left(\mathbb{F}_{p^{6}}\right) \text { and } u \in \mathscr{D}_{t} .\end{cases}
$$

Recall that $d(t)=3$ when $t \in C^{0}\left(\mathbb{F}_{p^{6}}\right)$. Combining Lemma 5.19 with Lemma 5.5 , and using Remark 5.6, we conclude the following.

Corollary 5.20. We have

$$
\begin{array}{r}
{\left[\operatorname{Aut}\left(\left(M_{2},\langle,\rangle_{2}\right)\right): \operatorname{Aut}((M,\langle,\rangle))\right]=\left[G_{\left(M_{2},\langle,\rangle_{2}\right)}: G_{(M,\langle,\rangle)}\right]=} \\
\begin{cases}2^{-e(p)} p^{3+2 d(t)}(p+1)\left(p^{2}-1\right)\left(p^{3}+1\right) & \text { if } u \notin \mathscr{D}_{t} \\
p^{3+2 d(t)}\left(p^{2}-1\right)\left(p^{3}+1\right) & \text { if } t \notin C^{0}\left(\mathbb{F}_{p^{6}}\right) \text { and } u \in \mathscr{D}_{t} \\
p^{9}(p+1)\left(p^{2}-1\right) & \text { if } t \in C^{0}\left(\mathbb{F}_{p^{6}}\right) \text { and } u \in \mathscr{D}_{t} .\end{cases}
\end{array}
$$

Now Corollary 2.5(1) and Corollary 5.20 yield the main result of this section, i.e., the mass formula for a supersingular principally polarised abelian threefold $x=(X, \lambda)$ of $a$-number 1, cf. Theorem B.

Theorem 5.21. Let $x=(X, \lambda) \in \mathscr{S}_{3,1}$ such that $a(X)=1$. For $\mu \in P^{1}\left(E^{3}\right)$, consider the associated polarised flag type quotient $\left(Y_{2}, \mu\right) \rightarrow\left(Y_{1}, \lambda_{1}\right) \rightarrow(X, \lambda)$ which is characterised by the pair $(t, u)$ with $t=\left(t_{1}: t_{2}: t_{3}\right) \in C^{0}(k)$ and $u=\left(u_{1}: u_{2}\right) \in \mathbb{P}^{1}(k)$. Let $\left(M_{2},\langle,\rangle_{2}\right)$ and $(M,\langle\rangle$,$) be the respective polarised Dieudonné modules of Y_{2}$ and $X$, let $\mathscr{D}_{t}$ be as in Definition 5.16, and let $d(t)$ be as in Definition 5.12. Then

$$
\begin{gathered}
\operatorname{Mass}\left(\Lambda_{x}\right)=\operatorname{Mass}\left(\Lambda_{3,1}\right) \cdot\left[\operatorname{Aut}\left(\left(M_{2},\langle,\rangle_{2}\right)\right): \operatorname{Aut}((M,\langle,\rangle))\right]= \\
\frac{p^{3}}{2^{10} \cdot 3^{4} \cdot 5 \cdot 7} \begin{cases}2^{-e(p)} p^{2 d(t)}\left(p^{2}-1\right)\left(p^{4}-1\right)\left(p^{6}-1\right) & \text { if } u \notin \mathscr{D}_{t} ; \\
p^{2 d(t)}(p-1)\left(p^{4}-1\right)\left(p^{6}-1\right) & \text { if } t \notin C^{0}\left(\mathbb{F}_{p^{6}}\right) \text { and } u \in \mathscr{D}_{t} ; \\
p^{6}\left(p^{2}-1\right)\left(p^{3}-1\right)\left(p^{4}-1\right) & \text { if } t \in C^{0}\left(\mathbb{F}_{p^{6}}\right) \text { and } u \in \mathscr{D}_{t} .\end{cases}
\end{gathered}
$$

\section{The automorphism groups}

In this section we discuss the automorphism groups of principally polarised abelian threefolds $(X, \lambda)$ over an algebraically closed field $k \supseteq \mathbb{F}_{p}$ with $a(X)=1$. We shall first focus on an open dense locus in $\mathscr{P}_{\mu}(a=1)$ (the $a$-number one locus in $\mathscr{P}_{\mu}$ ) in Subsection 6.2 and then discuss a few other cases in Subsections 6.3 and 6.4. To get started, we record some preliminaries in the next subsection. 


\subsection{Arithmetic properties of definite quaternion algebras over $\mathbb{Q}$}

Let $C_{n}$ denote the cyclic group of order $n \geq 1$. Let $B_{p, \infty}$ denote the definite quaternion $\mathbb{Q}$-algebra ramified exactly at $\{\infty, p\}$. The class number $h\left(B_{p, \infty}\right)$ of $B_{p, \infty}$ was determined by Deuring, Eichler and Igusa (cf. [10]) as follows:

$$
h\left(B_{p, \infty}\right)=\frac{p-1}{12}+\frac{1}{3}\left(1-\left(\frac{-3}{p}\right)\right)+\frac{1}{4}\left(1-\left(\frac{-4}{p}\right)\right),
$$

where $(\cdot / p)$ is the Legendre symbol. If $h\left(B_{p, \infty}\right)=1$, then the type number of $B_{p, \infty}$ is one and hence all maximal orders are conjugate. It follows from (54) that

$$
h\left(B_{p, \infty}\right)=1 \Longleftrightarrow p \in\{2,3,5,7,13\}
$$

If $p=2$, the quaternion algebra $B_{2, \infty} \simeq\left(\frac{-1,-1}{\mathbb{Q}}\right)$ is generated by $i, j$ with relations $i^{2}=j^{2}=-1$ and $k:=i j=-j i$, and the $\mathbb{Z}$-lattice

$$
O_{2, \infty}:=\operatorname{Span}_{\mathbb{Z}}\left\{1, i, j, \frac{1+i+j+k}{2}\right\}
$$

is a maximal order of $B_{2, \infty}$. Moreover,

$$
O_{2, \infty}^{\times}=\left\{ \pm 1, \pm i, \pm j, \pm k, \frac{ \pm 1 \pm i \pm j \pm k}{2}\right\}=: E_{24}
$$

and one has $E_{24} \simeq \mathrm{SL}_{2}\left(\mathbb{F}_{3}\right)$ and $E_{24} /\{ \pm 1\} \simeq A_{4}$.

If $p=3$, the quaternion algebra $B_{3, \infty} \simeq\left(\frac{-1,-3}{\mathbb{Q}}\right)$ is generated by $i, j$ with relations $i^{2}=-1, j^{2}=-3$ and $k:=i j=-j i$, and the $\mathbb{Z}$-lattice

$$
O_{3, \infty}:=\operatorname{Span}_{\mathbb{Z}}\left\{1, i, \frac{1+j}{2}, \frac{i(1+j)}{2}\right\}
$$

is a maximal order of $B_{3, \infty}$. Moreover,

$$
O_{3, \infty}^{\times}=\left\langle i, \zeta_{6}\right\rangle=: T_{12}, \quad \zeta_{6}=(1+j) / 2,
$$

and one has $T_{12} \simeq C_{4} \rtimes C_{3}$ and $T_{12} /\{ \pm 1\} \simeq D_{3}$, the dihedral group of order six.

If $p \geq 5$, then $O^{\times} \in\left\{C_{2}, C_{4}, C_{6}\right\}$ for any maximal order $O$ in $B_{p, \infty}$ [19, V Proposition 3.1, p. 145]. Fix a maximal order $O$ in $B_{p, \infty}$ and let $h\left(O, C_{2 n}\right)$ be the number of right $O$-ideal classes $[I]$ with $O_{\ell}(I)^{\times} \simeq C_{2 n}$, where $O_{\ell}(I)$ is the left order of $I$. Then (see [10])

$$
h\left(O, C_{4}\right)=\frac{1}{2}\left(1-\left(\frac{-4}{p}\right)\right) \quad \text { and } \quad h\left(O, C_{6}\right)=\frac{1}{2}\left(1-\left(\frac{-3}{p}\right)\right) .
$$




\section{Lemma 6.1.}

(1) Let $Q$ be a definite quaternion $\mathbb{Q}$-algebra and $O$ a $\mathbb{Z}$-order in $Q$, and let $n \geq 1$ be a positive integer. Then the integral quaternion hermitian group $U(n, O)=\{A \in$ $\left.\operatorname{Mat}_{n}(O): A \cdot A^{*}=\mathbb{I}_{n}\right\}$ is equal to the permutation unit group $\operatorname{diag}\left(O^{\times}, \ldots, O^{\times}\right) \cdot S_{n}$.

(2) Let $O$ be a maximal order in $B_{2, \infty}$. Let $m_{2}: U(n, O) \rightarrow \mathrm{GL}_{n}(O) \rightarrow \mathrm{GL}_{n}(O / 2 O)$ be the reduction-modulo-2 map. Then $\operatorname{ker}\left(m_{2}\right)=\operatorname{diag}(\{ \pm 1\}, \ldots,\{ \pm 1\}) \simeq C_{2}^{n}$.

Proof. (1) Note that $O$ is stable under the involution $*$ since $x^{*}=\operatorname{Tr} x-x$ and $\operatorname{Tr} x \in \mathbb{Z}$ for any $x$ in $O$. Let $A=\left(a_{i j}\right) \in U(n, O)$. Then since $A A^{*}=\mathbb{I}_{n}$, we have $\sum_{k} a_{i k} a_{i k}^{*}=$ 1 for any $1 \leq i \leq n$. Since $a_{i k} a_{i k}^{*}=0$ or 1 , for any $1 \leq i \leq n$, there is only one integer $1 \leq k \leq n$ such that $a_{i k} \neq 0$ and $a_{i k} \in O^{\times}$. On the other hand, since $A^{*} A=\mathbb{I}_{n}$, for any $1 \leq k \leq n$, there is a only one integer $1 \leq i \leq n$ such that $a_{i k} \neq 0$ and $a_{i k} \in O^{\times}$. Thus, $A \in \operatorname{diag}\left(O^{\times}, \ldots, O^{\times}\right) \cdot S_{n}$. Checking the reverse containment $\operatorname{diag}\left(O^{\times}, \ldots, O^{\times}\right) \cdot S_{n} \subseteq U(n, O)$ is straightforward.

(2) By (55), we may assume that $O=O_{2, \infty}$. Since the diagonal entries of elements in $\operatorname{ker}\left(m_{2}\right)$ are all not zero, by part (1) we find $\operatorname{ker}\left(m_{2}\right) \subseteq \operatorname{diag}\left(O^{\times}, \ldots, O^{\times}\right)$. Therefore, it suffices to show that the kernel of the reduction-modulo-2 map $m_{2}: O^{\times} \rightarrow$ $(O / 2 O)^{\times}$is isomorphic to $C_{2}$. Using (57) and $2 O=\left\{a_{1}+a_{2} i+a_{3} j+a_{4} k: a_{i} \in\right.$ $\left.\mathbb{Z}, a_{1} \equiv a_{2} \equiv a_{3} \equiv a_{4}(\bmod 2)\right\}$, one checks that indeed $\operatorname{ker}\left(m_{2}\right)=\{ \pm 1\} \subseteq O^{\times}$.

Lemma 6.2. Let $D_{p}$ be the quaternion division $\mathbb{Q}_{p}$-algebra and $O_{p}$ its maximal order. Let $n \geq 1$ be a positive integer. Let $\Pi$ be a uniformiser of $O_{p}$, and put $V_{p}:=1+\Pi \operatorname{Mat}_{n}\left(O_{p}\right) \subseteq$ $\mathrm{GL}_{n}\left(O_{p}\right)$. If $p \geq 5$, then the torsion subgroup $\left(V_{p}\right)_{\text {tors }}$ of $V_{p}$ is trivial.

Remark 6.3. Before giving the proof, let us note that $p \geq 5$ is best possible. Indeed, when $p=3$, we have

$$
D_{3}=\left(\frac{-1,-3}{\mathbb{Q}_{3}}\right), \quad O_{3}=\mathbb{Z}_{3}[i,(1+j) / 2]=\mathbb{Z}_{3}[i, j], \quad \Pi=j .
$$

Thus, we find the torsion element $-(1+j) / 2 \in 1+\Pi O_{p}$.

Proof of Lemma 6.2. For simplicity, write $(\Pi)$ for the two-sided ideal in $\operatorname{Mat}_{n}\left(O_{p}\right)$ generated by $\Pi$. We must show that any $\alpha \in\left(V_{p}\right)_{\text {tor }}$ must equal 1 . Since $V_{p}$ is a pro- $p$ group, we have $\alpha^{p^{r}}=1$ for some $r \geq 1$. By induction, we may assume that $\alpha^{p}=1$. Suppose that $\alpha \neq 1$ and write $\alpha=1+\Pi \beta$ for some nonzero $\beta \in \operatorname{Mat}_{n}\left(O_{p}\right)$. Necessarily, $\beta \notin(\Pi)$, for otherwise $\alpha \equiv 1(\bmod p)$, which implies that $\alpha=1$ by a lemma of Serre [14, p. 207]. Since $p \geq 5$ and $p \mid\left(\begin{array}{c}p \\ i\end{array}\right)$ for all $1 \leq i \leq p-1$, we find

$$
1=\sum_{i=0}^{p}\left(\begin{array}{c}
p \\
i
\end{array}\right)(\Pi \beta)^{i} \equiv 1+p \Pi \beta \quad\left(\bmod \Pi^{4}\right)
$$


This implies that $\beta \in(\Pi)$, which leads to a contradiction.

\subsection{The region outside the divisor $\mathscr{D}$}

Recall from Subsection 3.1 that $E$ is a supersingular elliptic curve over $\mathbb{F}_{p^{2}}$ such that $\pi_{E}=-p$. Let $\mu_{\text {can }} \in P\left(E^{3}\right)$ be the threefold self-product of the canonical principal polarisation on $E$; this is also called the canonical polarisation on $E^{3}$.

Theorem 6.4. Let $x=(X, \lambda) \in \mathscr{S}_{3,1}(k)$ with $a(X)=1$. For $\mu \in P\left(E^{3}\right)$, consider the associated polarised flag type quotient $\left(Y_{2}, p \mu\right) \rightarrow\left(Y_{1}, \lambda_{1}\right) \rightarrow(X, \lambda)$ which is characterised by the pair $(t, u)$ with $t=\left(t_{1}: t_{2}: t_{3}\right) \in C^{0}(k)$ and $u=\left(u_{1}: u_{2}\right) \in \mathbb{P}^{1}(k)$. Let $\left(M_{2},\langle,\rangle_{2}\right)$ and $(M,\langle\rangle$,$) be the respective polarised Dieudonné modules of \left(Y_{2}, \mu\right)$ and $(X, \lambda)$, let $\mathscr{D}_{t}$ be as in Definition 5.16 and let $d(t)$ be as in Definition 5.12. Assume that $(t, u) \notin \mathscr{D}$, that is, $u \notin \mathscr{D}_{t}$.

(1) If $p=2$, then $\operatorname{Aut}(X, \lambda) \simeq C_{2}^{3}$.

(2) If $p \geq 5$, or $p=3$ and $d(t)=6$, then $\operatorname{Aut}(X, \lambda) \simeq C_{2}$.

Proof. By Proposition 3.16, $\left(Y_{2}, p \mu\right) \rightarrow(X, \lambda)$ is the minimal isogeny. Therefore,

$$
\operatorname{Aut}(X, \lambda)=\left\{h \in \operatorname{Aut}\left(Y_{2}, \mu\right): m_{p}(h) \in G_{(M,\langle,\rangle)}\right\}
$$

By Proposition 5.10, we have an exact sequence

$$
1 \rightarrow \operatorname{ker}\left(\psi_{t}\right) \rightarrow G_{(M,\langle,\rangle)} \stackrel{m_{\Pi}}{\longrightarrow} \bar{G}_{(M,\langle,\rangle)} \rightarrow 1
$$

(1) A direct calculation using the mass formula (cf. Corollary 2.5 and Lemma 6.1) shows

$$
\operatorname{Mass}\left(\Lambda_{3,1}\right)=\frac{1}{2^{10} \cdot 3^{4}}=\frac{1}{24^{3} \cdot 3 !}=\frac{1}{\left|\operatorname{Aut}\left(E^{3}, \mu_{\text {can }}\right)\right|},
$$

and hence $\left|\Lambda_{3,1}\right|=1$. Thus, we may assume that $\left(Y_{2}, \mu\right)=\left(E^{3}, \mu_{\text {can }}\right)$, and we have $\operatorname{Aut}\left(Y_{2}, \mu\right)=\operatorname{diag}\left(O^{\times}, O^{\times}, O^{\times}\right) \cdot S_{3}$ by Lemma 6.1 with $O=\operatorname{End}(E)$. As $u \notin \mathscr{D}_{t}$, Corollary 5.18 yields $\bar{G}_{(M,\langle,\rangle)}=\{ \pm 1\}=1$. We see from the proof of Proposition 5.13

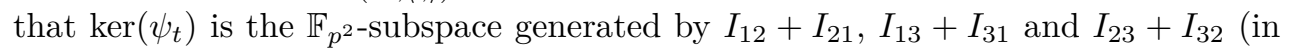
the notation of that proof). Therefore,

$$
G_{(M,\langle,\rangle)}=\left\{\left(\begin{array}{cc}
\mathbb{I}_{3} & 0 \\
S & \mathbb{I}_{3}
\end{array}\right): S=\left(s_{i j}\right) \in S_{3}\left(\mathbb{F}_{p^{2}}\right), s_{i i}=0 \forall 1 \leq i \leq 3\right\} .
$$

Let $h \in \operatorname{Aut}(X, \lambda) \subseteq \operatorname{diag}\left(O^{\times}, O^{\times}, O^{\times}\right) \cdot S_{3}$. Since $m_{2}(h)$ has non-zero diagonal entries, $h \in \operatorname{diag}\left(O^{\times}, O^{\times}, O^{\times}\right)$. One deduces $m_{2}(h)=1$ from (64). Thus, $h \in \operatorname{ker}\left(m_{2}\right)=C_{2}^{3}$, by Lemma 6.1. On the other hand, $\operatorname{ker}\left(m_{2}\right) \subseteq \operatorname{Aut}(X, \lambda)$ from (62). This proves (1). 
(2) Assume $p \geq 5$. As $u \notin \mathscr{D}_{t}$, Corollary 5.18 implies that $\bar{G}_{(M,\langle,\rangle)}=\{ \pm 1\}$. Lemma 6.2 implies that the map $m_{\Pi}: \operatorname{Aut}(X, \lambda) \rightarrow \bar{G}_{(M,\langle,\rangle)}$ is injective, because $\operatorname{ker}\left(m_{\Pi}\right)$ is contained in $\left(V_{p}\right)_{\text {tors }}$. Thus, $\operatorname{Aut}(X, \lambda) \simeq C_{2}$. Now assume $p=3$ and $d(t)=6$. In this case $G_{(M,\langle,\rangle)}=\{ \pm 1\}$ follows from (63) and Corollary 5.18. By a lemma of Serre [14, p. 207], the map $m_{3}: \operatorname{Aut}(X, \lambda) \rightarrow G_{(M,\langle,\rangle)}$ is injective and hence $\operatorname{Aut}(X, \lambda) \simeq C_{2}$.

Corollary 6.5. Let the notation and assumptions be as in Theorem 6.4.

(1) If $p=2$, then $\left|\Lambda_{x}\right|=4$.

(2) If $p=3$ and $d(t)=6$, then $\left|\Lambda_{x}\right|=3^{11} \cdot 13$.

(3) If $p \geq 5$, then

$$
\left|\Lambda_{x}\right|=\frac{p^{3+2 d(t)}\left(p^{2}-1\right)\left(p^{4}-1\right)\left(p^{6}-1\right)}{2^{10} \cdot 3^{4} \cdot 5 \cdot 7}
$$

Proof. All statements follow from Theorems 5.21 and 6.4. For $p=2$, we have $\operatorname{Aut}(X, \lambda) \simeq C_{2}^{3}$ for each $(X, \lambda) \in \Lambda_{x}$ and hence

$$
\left|\Lambda_{x}\right|=\frac{2^{3} \cdot 2^{9} \cdot 3 \cdot(3 \cdot 5) \cdot\left(3^{2} \cdot 7\right)}{2^{10} \cdot 3^{4} \cdot 5 \cdot 7}=4
$$

For $p=3$ and $d(t)=6$, we have $\operatorname{Aut}(X, \lambda) \simeq C_{2}$ for each $(X, \lambda) \in \Lambda_{x}$ and hence

$$
\left|\Lambda_{x}\right|=\frac{3^{3+2 d(t)} \cdot 2^{3} \cdot\left(2^{4} \cdot 5\right) \cdot\left(2^{3} \cdot 7 \cdot 13\right)}{2^{10} \cdot 3^{4} \cdot 5 \cdot 7}=3^{2 d(t)-1} \cdot 13=3^{11} \cdot 13
$$

The same argument gives (65) for $p \geq 5$.

A $g$-dimensional principally polarised supersingular abelian variety $(X, \lambda)$ over $k$ is said to be generic if the moduli point $\operatorname{Spec} k \rightarrow \mathscr{S}_{g, 1}$ factors through a generic point of $\mathscr{S}_{g, 1}$. Recall that the supersingular locus $\mathscr{S}_{g, 1} \subseteq \mathscr{A}_{g, 1} \otimes \overline{\mathbb{F}}_{p}$ is a scheme of finite type over $\overline{\mathbb{F}}_{p}$ which is defined over $\mathbb{F}_{p}$. Moreover, every geometrically irreducible component of $\mathscr{S}_{g, 1}$ is defined over $\mathbb{F}_{p^{2}}$, cf. [26, Section 2.2].

Oort's conjecture [2, Problem 4] asserts that for any integer $g \geq 2$ and any prime number $p$, every generic $g$-dimensional principally polarised supersingular abelian variety $(X, \lambda)$ over $k$ of characteristic $p$ has automorphism group $\{ \pm 1\}$. Oort's conjecture fails with counterexamples in $(g, p)=(2,2)$ or $(g, p)=(3,2)$; see $[8,16]$.

For fixed $g \geq 2$ and prime number $p$, consider the refined Oort conjecture:

$(\mathrm{O})_{g, p}$ : Every generic $g$-dimensional principally polarised supersingular abelian variety $(X, \lambda)$ over $k$ of characteristic $p$ has automorphism group $\{ \pm 1\}$. 
Corollary 6.6. Let $(X, \lambda)$ be a generic principally polarised supersingular abelian threefold over $k$ of characteristic $p>0$. Then

$$
\operatorname{Aut}(X, \lambda) \simeq \begin{cases}C_{2}^{3} & \text { for } p=2 \\ C_{2} & \text { for } p \geq 3\end{cases}
$$

Proof. This follows immediately from Theorem 6.4.

In other words, Oort's Conjecture $(\mathrm{O})_{3, p}$ holds precisely when $p \neq 2$.

\section{Remark 6.7.}

(1) It is shown [16, Theorem 5.6, p. 270] that if $(X, \lambda)$ is a principally polarised supersingular abelian threefold over $k$ of characteristic 2 , then $\operatorname{Aut}(X, \lambda) \supseteq C_{2}^{3}$. By Corollary 6.6, the smallest group $C_{2}^{3}$ also appears as $\operatorname{Aut}(X, \lambda)$ for some $(X, \lambda)$. We have seen that the unique member $\left(E^{3}, \mu_{\text {can }}\right)$ in $\Lambda_{3,1}$ has automorphism group $E_{24}^{3} \rtimes S_{3}$ (of order $2^{10} \cdot 3^{4}$ ). We expect that $2^{10} \cdot 3^{4}$ is the maximal order of automorphism groups of all principally polarised abelian threefolds over $k$ of any characteristic (including zero).

(2) According to Hashimoto's result [5], we have $\left|\Lambda_{3,1}\right|=2$ for $p=3$. In this case, we have two isomorphism classes, represented by $\left(E^{3}, \mu_{\text {can }}\right)$ and $\left(E^{3}, \mu\right)$. Using Lemma 6.1, we compute $\left|\operatorname{Aut}\left(E^{3}, \mu_{\text {can }}\right)\right|=2^{7} \cdot 3^{4}$ and conclude $\left|\operatorname{Aut}\left(E^{3}, \mu\right)\right|=2^{7} \cdot 3^{4}$ from the mass formula $\operatorname{Mass}\left(\Lambda_{3,1}\right)=1 /\left(2^{6} \cdot 3^{4}\right)$.

\subsection{The region where $t \notin C\left(\mathbb{F}_{p^{6}}\right)$ and $(t, u) \in \mathscr{D}$}

In this subsection we consider the region $(t, u) \in \mathscr{D}$ and assume that $t \notin C\left(\mathbb{F}_{p^{6}}\right)$. This extends the region considered in Subsection 6.2.

Lemma 6.8. Let $(X, \lambda) \in \mathscr{S}_{3,1}(k)$ with $a(X)=1$. If $p \geq 3$ and $\operatorname{Aut}(X, \lambda) \subseteq C_{p+1}$, then $\operatorname{Aut}(X, \lambda) \subseteq\left\{C_{2}, C_{4}, C_{6}\right\}$.

Proof. Suppose that $\operatorname{Aut}(X, \lambda)=C_{2 d}$ with $2 d \mid(p+1)$. Then we have a ring homomorphism $\mathbb{Z}\left[C_{2 d}\right] \rightarrow \operatorname{End}(X)$ which maps $C_{2 d}$ bijectively to $\operatorname{Aut}(X, \lambda)$. The $\mathbb{Q}$-algebra homomorphism

$$
\mathbb{Q}\left[C_{2 d}\right]=\prod_{d^{\prime} \mid 2 d} \mathbb{Q}\left[\zeta_{d^{\prime}}\right] \rightarrow \operatorname{End}^{0}(X)=\operatorname{Mat}_{3}\left(B_{p, \infty}\right)
$$

factors through an injective $\mathbb{Q}$-algebra homomorphism

$$
\prod_{i=1}^{r} \mathbb{Q}\left[\zeta_{d_{i}}\right] \hookrightarrow \operatorname{End}^{0}(X)=\operatorname{Mat}_{3}\left(B_{p, \infty}\right),
$$


where $\left\{d_{i} \mid 2 d\right\} \subseteq\left\{d^{\prime} \mid 2 d\right\}$. Since the composition gives an embedding $C_{2 d} \hookrightarrow \operatorname{Aut}(X)$, the integers $\left\{d_{i}\right\}$ satisfy $\operatorname{lcm}\left(d_{1}, \ldots, d_{r}\right)=2 d$. Since $p \nmid 2 d$, the algebra $\mathbb{Z}_{p}\left[C_{2 d}\right]$ is étale over $\mathbb{Z}_{p}$ and is the maximal order in $\mathbb{Q}_{p}\left[C_{2 d}\right]$. This gives rise to an embedding $\prod_{i=1}^{r} \mathbb{Z}\left[\zeta_{d_{i}}\right] \otimes$ $\mathbb{Z}_{p} \hookrightarrow \operatorname{End}(X) \otimes \mathbb{Z}_{p} \simeq \operatorname{End}\left(X\left[p^{\infty}\right]\right)$. Thus, the decomposition $X\left[p^{\infty}\right]=H_{1} \times \cdots \times H_{r}$ into a product of supersingular $p$-divisible groups shows $a(X) \geq r$ and hence $r=1$. Therefore, there is a $\mathbb{Q}$-algebra embedding of $\mathbb{Q}\left(\zeta_{2 d}\right)$ into $\operatorname{Mat}_{3}\left(B_{p, \infty}\right)$. This implies that $\varphi(2 d) \mid 6$ (where $\varphi$ denotes Euler's totient function) and hence $2 d \in\{2,4,6,14,18\}$.

If $2 d=14$, then $p \equiv-1(\bmod 7)$ and $\operatorname{ord}(p)=2$ in $(\mathbb{Z} / 7 \mathbb{Z})^{\times}$. This gives rise to an embedding $\mathbb{Z}\left[\zeta_{14}\right] \otimes \mathbb{Z}_{p}=\mathbb{Z}_{p^{2}} \times \mathbb{Z}_{p^{2}} \times \mathbb{Z}_{p^{2}} \hookrightarrow \operatorname{End}\left(X\left[p^{\infty}\right]\right)$ and hence $a(X)=3$, a contradiction. If $2 d=18$, then $p \equiv-1(\bmod 9)$ and $\operatorname{ord}(p)=2$ in $(\mathbb{Z} / 9 \mathbb{Z})^{\times}$. Similarly, we get an embedding $\mathbb{Z}\left[\zeta_{18}\right] \otimes \mathbb{Z}_{p}=\mathbb{Z}_{p^{2}} \times \mathbb{Z}_{p^{2}} \times \mathbb{Z}_{p^{2}} \hookrightarrow \operatorname{End}\left(X\left[p^{\infty}\right]\right)$ and $a(X)=3$, again a contradiction.

Recall that $\mathbb{F}_{p^{2}}^{1}:=\left\{\alpha \in \mathbb{F}_{p^{2}}^{\times}: \alpha^{p+1}=1\right\} \simeq C_{p+1}$ denotes the group of norm one elements in $\mathbb{F}_{p^{2}}^{\times}$.

Theorem 6.9. Let the notation be as in Theorem 6.4. Assume that $(t, u) \in \mathscr{D}$ and $t \notin$ $C\left(\mathbb{F}_{p^{6}}\right)$.

(1) If $p=2$, then $\operatorname{Aut}(X, \lambda) \simeq C_{2}^{3} \times C_{3}$.

(2) If $p=3$ and $d(t)=6$, then $\operatorname{Aut}(X, \lambda) \in\left\{C_{2}, C_{4}\right\}$.

(3) For $p \geq 5$, we have the following cases:

(i) If $p \equiv-1(\bmod 4)$, then $\operatorname{Aut}(X, \lambda) \in\left\{C_{2}, C_{4}\right\}$.

(ii) If $p \equiv-1(\bmod 3)$, then $\operatorname{Aut}(X, \lambda) \in\left\{C_{2}, C_{6}\right\}$.

(iii) If $p \equiv 1(\bmod 12)$, then $\operatorname{Aut}(X, \lambda) \simeq C_{2}$.

Proof. (1) As in Theorem 6.4(1), we may assume that $\left(Y_{2}, \mu\right)=\left(E^{3}, \mu_{\text {can }}\right)$, and by Lemma 6.1 we have $\operatorname{Aut}\left(Y_{2}, \mu\right)=\operatorname{diag}\left(O^{\times}, O^{\times}, O^{\times}\right) \cdot S_{3}$. Then

$$
\begin{aligned}
& \operatorname{Aut}(X, \lambda)=\left\{h \in \operatorname{Aut}\left(Y_{2}, \mu\right): m_{2}(h)=\left(\begin{array}{lll}
a & & \\
& a & \\
& & a
\end{array}\right), a \in \mathbb{F}_{4}^{1}\right\} \\
& =\left\{h \in \operatorname{diag}\left(O^{\times}, O^{\times}, O^{\times}\right): m_{2}(h)=\left(\begin{array}{ccc}
a & & \\
& a & \\
& & a
\end{array}\right), a \in \mathbb{F}_{4}^{1}\right\} \\
& =\left\{\left(\begin{array}{lll} 
\pm w^{j} & & \\
& \pm w^{j} & \\
& & \pm w^{j}
\end{array}\right): 0 \leq j \leq 5\right\} \simeq C_{2}^{3} \times C_{3},
\end{aligned}
$$

where $w=(1+i+j+k) / 2$ satisfies $w^{6}=1$.

(2) In this case, $\bar{G}_{(M,\langle,\rangle)}=\mathbb{F}_{9}^{1} \simeq C_{4}$ by Corollary 5.18. The proof then follows from the fact that the reduction-modulo-3 map is injective. 
(3) In this case, $\bar{G}_{(M,\langle,\rangle)}=\mathbb{F}_{p^{2}}^{1} \simeq C_{p+1}$ by Corollary 5.18. It follows from Lemma 6.2 that $\operatorname{Aut}(X, \lambda)$ can be identified with a subgroup of $\bar{G}_{(M,\langle,\rangle)} \simeq C_{p+1}$ as $p \geq 5$. By Lemma 6.8, $\operatorname{Aut}(X, \lambda) \in\left\{C_{2}, C_{4}, C_{6}\right\}$. The assertions for (i), (ii), (iii) and (iv) follow from this assertion.

Write $\mathscr{D}_{\mu}$ for $\mathscr{D} \subseteq \mathscr{P}_{\mu}(a=1)$ to emphasise its dependence on $\mu \in P\left(E^{3}\right)$. Recall that

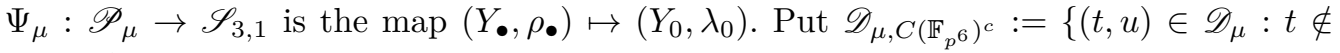
$\left.C\left(\mathbb{F}_{p^{6}}\right)\right\}$.

Let $\Lambda_{1}$ denote the set of $\mathbb{F}_{p^{2}}$-isomorphism classes of supersingular elliptic curves $E^{\prime}$ over $\mathbb{F}_{p^{2}}$ with Frobenius endomorphism $\pi_{E^{\prime}}=-p$. This set is in bijection with the set $\mathrm{Cl}\left(B_{p, \infty}\right)$ of right $O$-ideal classes for a fixed maximal order $O$ in $B_{p, \infty}$; see [1] (also cf. [20, Theorem 2.1]).

\section{Proposition 6.10.}

(1) If $p=3$ and $d(t)=6$, then for all $(X, \lambda) \in \Psi_{\mu}\left(\mathscr{D}_{\mu, C\left(\mathbb{F}_{p^{6}}\right)^{c}}\right)$ with $\mu=\mu_{\text {can }}$, one has $\operatorname{Aut}(X, \lambda) \simeq C_{4}$.

(2) If $p \geq 5$ and $p \equiv 3(\bmod 4)$, then there exists $\mu \in P\left(E^{3}\right)$ such that for all $(X, \lambda) \in$

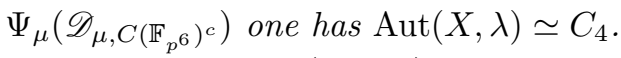

(3) If $p \geq 5$ and $p \equiv 2(\bmod 3)$, then there exists $\mu \in P\left(E^{3}\right)$ such that for all $(X, \lambda) \in$

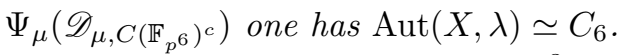

(4) If $p \geq 11$, then there exists $\mu \in P\left(E^{3}\right)$ such that for all $(X, \lambda) \in \Psi_{\mu}\left(\mathscr{D}_{\mu, C\left(\mathbb{F}_{p^{6}}\right)^{c}}\right)$ one has $\operatorname{Aut}(X, \lambda) \simeq C_{2}$.

Proof. We use the results from Subsection 6.1. If $p=3$, then $O^{\times}=\operatorname{Aut}(E)=\left\langle i, \zeta_{6}\right\rangle$. If $p \geq 5$ and $p \equiv 2(\bmod 3)(\operatorname{resp} . p \equiv 3(\bmod 4))$, there exists a unique supersingular elliptic curve $E^{\prime}$ in $\Lambda_{1}$ such that $O^{\times}:=\operatorname{Aut}\left(E^{\prime}\right) \simeq C_{6}$ (resp. $\left.C_{4}\right)$. If $p \geq 11$, then there exists a supersingular elliptic curve $E^{\prime}$ in $\Lambda_{1}$ such that $O^{\times}:=\operatorname{Aut}\left(E^{\prime}\right) \simeq C_{2}$. Note that if $p \geq 11$ then either $h\left(B_{p, \infty}\right) \geq 2$ or $p \equiv 1(\bmod 12)$. For cases $(2),(3)$, and (4) we choose a polarisation $\mu \in P\left(E^{3}\right)$ such that $\left(E^{3}, \mu\right) \simeq\left(E^{\prime 3}, \mu_{\text {can }}^{\prime}\right)$, where $\mu_{\text {can }}^{\prime}$ is the canonical polarisation on $E^{\prime 3}$ as before. (In case (1) $\mu=\mu_{\text {can }}$ is the unique choice of polarisation.) Then using the same argument as in Theorem 6.9, the automorphism $\operatorname{group} \operatorname{Aut}(X, \lambda)$ for $(X, \lambda) \in \Psi_{\mu}\left(\mathscr{D}_{\mu, C\left(\mathbb{F}_{p^{6}}\right)^{c}}\right)$ consists of elements of the form $\operatorname{diag}(a, a, a)$ with $a \in O^{\times}$satisfying $m_{3}(a) \in \mathbb{F}_{4}^{1}$ if $p=3$ (resp. $m_{\Pi}(a) \in \mathbb{F}_{p^{2}}^{1}$ if $p \geq 5$ ). If $p=3$, we have $m_{3}(\langle i\rangle)=C_{4}$. If $p \equiv 3(\bmod 4)$, we have $m_{\Pi}(\langle i\rangle)=C_{4}$. If $p \equiv 2(\bmod 3)$, we have $m_{\Pi}\left(\left\langle\zeta_{6}\right\rangle\right)=C_{6}$. Thus, $\operatorname{Aut}(X, \lambda) \simeq C_{4}$ for $p \equiv 3(\bmod 4)$ and $\operatorname{Aut}(X, \lambda) \simeq C_{6}$ for $p \equiv 2$ $(\bmod 3)$. In case $(4)$, we have $\operatorname{Aut}(X, \lambda) \simeq C_{2}$.

\section{Remark 6.11.}

(1) Given Proposition 6.10, it remains to check whether the group $C_{2}$ also appears as $\operatorname{Aut}(X, \lambda)$ in the region $\Psi_{\mu}\left(\mathscr{D}_{\mu, C\left(\mathbb{F}_{p^{6}}\right)^{c}}\right)$ for some $\mu \in P\left(E^{3}\right)$ when $p=3,5,7$. 
(2) We assume the condition $d(t)=6$ when $p=3$ in Theorems 6.4 and 6.9. It remains to determine which other automorphism groups occur if this condition is dropped.

\subsection{The superspecial case}

As we have seen in the previous subsection, to investigate the automorphism groups in some special region of $\mathscr{P}_{\mu}(a=1)$, the knowledge of automorphism groups arising from the superspecial locus $\Lambda_{3,1}$ also plays an important role. In this subsection, we discuss only preliminary results on the automorphism groups of members in $\Lambda_{3,1}$. A complete list of all possible automorphism groups requires much more work; see Question (2) below.

We briefly recall some results. For $p=2$, we have $\left|\Lambda_{3,1}\right|=1$ and the unique isomorphism class represented by $(X, \lambda)$ has automorphism group $E_{24}^{3} \rtimes S_{3}$. For $p=3$, we have $\left|\Lambda_{3,1}\right|=2$ by Hashimoto's result. In this case, the two isomorphism classes are represented by $\left(E^{3}, \mu_{\text {can }}\right)$ and $\left(E^{3}, \mu\right)$, respectively, and we have $\operatorname{Aut}\left(E^{3}, \mu_{\text {can }}\right)=T_{12}^{3} \rtimes S_{3}$ so $\left|\operatorname{Aut}\left(E^{3}, \mu\right)\right|=2^{7} \cdot 3^{4}$, cf. Remark 6.7. For $p \geq 5$, the following non-abelian groups occur:

$$
\begin{cases}C_{2}^{3} \rtimes S_{3} & \text { for } p \equiv 1(\bmod 12) ; \\ C_{4}^{3} \rtimes S_{3} & \text { for } p \equiv 3(\bmod 4) ; \\ C_{6}^{3} \rtimes S_{3} & \text { for } p \equiv 2(\bmod 6),\end{cases}
$$

\section{cf. Lemma 6.1.}

Unlike the $a$-number one case, it is more difficult to construct a member $(X, \lambda)$ in $\Lambda_{3,1}$ such that $\operatorname{Aut}(X, \lambda) \simeq C_{2}$. However, it is expected that when $p$ goes to infinity, most members of $\Lambda_{g, 1}$ have automorphism group $C_{2}$. The following result confirms this expectation for $g=3$, based on Hashimoto's result [5].

Proposition 6.12. Let $\Lambda_{3,1}\left(C_{2}\right):=\left\{(X, \lambda) \in \Lambda_{3,1}: \operatorname{Aut}(X, \lambda) \simeq C_{2}\right\}$. Then

$$
\frac{\left|\Lambda_{3,1}\left(C_{2}\right)\right|}{\left|\Lambda_{3,1}\right|} \rightarrow 1 \quad \text { as } p \rightarrow \infty
$$

Proof. Put $h_{2}(p):=\left|\Lambda_{3,1}\left(C_{2}\right)\right|$. By [5, Main Theorem], the main term of $h(p):=\left|\Lambda_{3,1}\right|$ is $H_{1}(p):=(p-1)\left(p^{2}+1\right)\left(p^{3}-1\right) /\left(2^{9} \cdot 3^{4} \cdot 5 \cdot 7\right)$ and the error term $\varepsilon(p)$ is $O\left(p^{5}\right)$. Observe that $\operatorname{Mass}\left(\Lambda_{3,1}\right)=H_{1}(p) / 2$. If $(X, \lambda) \notin \Lambda_{3,1}\left(C_{2}\right)$, then $|\operatorname{Aut}(X, \lambda)| \geq 4$. This gives the inequality

$$
\operatorname{Mass}\left(\Lambda_{3,1}\right) \leq \frac{h_{2}(p)}{2}+\frac{h(p)-h_{2}(p)}{4}=\frac{h_{2}(p)}{4}+\frac{H_{1}(p)+\varepsilon(p)}{4}
$$

From $\operatorname{Mass}\left(\Lambda_{3,1}\right)=H_{1}(p) / 2$ one deduces that $h_{2}(p) \geq H_{1}(p)-\varepsilon(p)$. Since

$$
\frac{H_{1}(p)-\varepsilon(p)}{H_{1}(p)+\varepsilon(p)} \leq \frac{\left|\Lambda_{3,1}\left(C_{2}\right)\right|}{\left|\Lambda_{3,1}\right|} \leq 1 \quad \text { and } \quad \frac{H_{1}(p)-\varepsilon(p)}{H_{1}(p)+\varepsilon(p)} \rightarrow 1 \quad \text { as } p \rightarrow \infty
$$


we get the assertion (68).

We end the paper with some open problems.

\section{Questions.}

(1) Let $X$ be a principally polarisable supersingular abelian variety over $k$, and let $P(X)$ be the set of isomorphism classes of principal polarisations on $X$. The mass of $P(X)$ is defined as

$$
\operatorname{Mass}(P(X)):=\sum_{\lambda \in P(X)} \frac{1}{|\operatorname{Aut}(X, \lambda)|} .
$$

One would like to find a mass formula for $\operatorname{Mass}(P(X))$ and understand the relationship between the sets $P(X)$ and $\Lambda_{(X, \lambda)}$ for a polarisation $\lambda \in P(X)$ when $\operatorname{dim}(X)=3$. Ibukiyama [8] studied $P(X)$ for $\operatorname{dim}(X)=2$. He gave a mass formula for $\operatorname{Mass}(P(X))$ and also showed that $P(X)$ is in bijection with the set $\Lambda_{(X, \lambda)}$ for any principal polarisation $\lambda$ on $X$. Note that not every supersingular abelian threefold is principally polarisable: by [13, Theorem 10.5, p. 71] we see that the supersingular locus $\mathscr{S}_{3, d} \subseteq \mathscr{A}_{3, d} \otimes \overline{\mathbb{F}}_{p}$ is three-dimensional if $d$ is divisible by a high power of $p$, while $\operatorname{dim}\left(\mathscr{S}_{3,1}\right)=2$.

(2) In order to study the automorphism groups of $(X, \lambda)$ with $a(X)=2$, we also need to study the automorphism groups arising from the non-principal genus $\Lambda_{3, p}$; see Proposition 3.16. Do we have an asymptotic result similar to Proposition 6.12 for $\Lambda_{3, p}$ ? What are the possible automorphism groups arising from $\Lambda_{3,1}$ or from $\Lambda_{3, p}$ ? We refer to Ibukiyama-Katsura-Oort [9], Katsura-Oort [11] and Ibukiyama [7] for detailed investigations for the principal genus case $\Lambda_{2,1}$ and the non-principal genus case $\Lambda_{2, p}$. Observe that there are natural maps $\Lambda_{2,1} \times \Lambda_{1,1} \rightarrow \Lambda_{3,1}$ and $\Lambda_{2, p} \times \Lambda_{1,1} \rightarrow$ $\Lambda_{3, p}$. Following the references mentioned above, these maps already produce many automorphism groups of members of $\Lambda_{3,1}$ and $\Lambda_{3, p}$.

(3) We say two polarised abelian varieties $\left(X_{1}, \lambda_{1}\right)$ and $\left(X_{2}, \lambda_{2}\right)$ are isogenous, denoted $\left(X_{1}, \lambda_{1}\right) \sim\left(X_{2}, \lambda_{2}\right)$, if there exists a quasi-isogeny $\varphi: X_{1} \rightarrow X_{2}$ such that $\varphi^{*} \lambda_{2}=\lambda_{1}$. Let $x=\left(X_{0}, \lambda_{0}\right) \in \mathscr{A}_{g, 1}(k)$ be a geometric point. Define

$$
\Lambda_{x}:=\left\{(X, \lambda) \in \mathscr{A}_{g, 1}(k):(X, \lambda) \sim\left(X_{0}, \lambda_{0}\right) \text { and }(X, \lambda)\left[p^{\infty}\right] \simeq\left(X_{0}, \lambda_{0}\right)\left[p^{\infty}\right]\right\}
$$

Using the foliation structure on Newton strata due to Oort [17], one can show that the set $\Lambda_{x}$ is finite. Note that any two principally polarised supersingular abelian varieties over $k$ are isogenous, cf. [21, Corollary 10.3]. Thus, the definition of $\Lambda_{x}$ in (70) coincides that of $\Lambda_{x}$ in (3) when $x \in \mathscr{S}_{g, 1}$. That is, a mass function

$$
\operatorname{Mass}: \mathscr{A}_{g, 1}(k) \rightarrow \mathbb{Q}, \quad x \mapsto \operatorname{Mass}\left(\Lambda_{x}\right)
$$


extends the mass function $\operatorname{Mass}(x):=\operatorname{Mass}\left(\Lambda_{x}\right)$ defined on $\mathscr{S}_{g, 1}(k)$ as before. One would like to compute or study the properties of such a mass function on $\mathscr{A}_{g, 1}(k)$, starting in low genus $g$. This problem may require developing more explicit descriptions of the foliation structure on Newton strata, or employing analogues of the Rapoport-Zink space which was introduced in Subsection 3.1.

\section{Acknowledgments}

Parts of this work were carried out when the first author visited the Academia Sinica, and when the first and third authors visited RIMS and Kyoto University. They would like to thank these institutes for their hospitality and excellent working conditions. A part of this paper is contained in the second author's master's thesis written at Tohoku University; he thanks his advisor Nobuo Tsuzuki for enlightening comments, advice and encouragement. The authors are grateful to Ming-Lun Hsieh and Akio Tamagawa for useful discussions, and for proving Propositions A.2 and A.3, respectively. They would like to thank Tomoyoshi Ibukiyama and Jiangwei Xue for useful discussions and helpful comments on an earlier manuscript, and the anonymous referee for their comments which improved the exposition. The second author is supported by JSPS grants 15J05073 and 19K14501. The third author is partially supported by MoST grants 107-2115-M-001-001MY2 and 109-2115-M-001-002-MY3.

\section{Appendix A. The intersection $C \cap \Delta$}

Let $C \subseteq \mathbb{P}^{2}$ be the Fermat curve defined by the equation $X_{1}^{p+1}+X_{2}^{p+1}+X_{3}^{p+1}=0$ and $\Delta \subseteq \mathbb{P}^{2}$ the curve defined in Proposition 5.13.

In Section 5 we have seen the inclusion

$$
C\left(\mathbb{F}_{p^{2}}\right) \coprod C^{0}\left(\mathbb{F}_{p^{6}}\right) \coprod C^{0}\left(\mathbb{F}_{p^{8}}\right) \coprod C^{0}\left(\mathbb{F}_{p^{10}}\right) \subseteq C \cap \Delta
$$

for $p>2$. In this (independent) section we study the complement of this inclusion.

\section{A.1. Bounds for the degrees}

Let $\mathscr{Q}$ denote the set of all conics (including degenerate ones) $Q \subseteq \mathbb{P}^{2}$ defined over $\mathbb{F}_{p^{2}}$. Then $\Delta=\cup_{Q \in \mathscr{Q}} Q$. If $t \in C \cap \Delta$, then $t \in C \cap Q$ for some $Q \in \mathscr{Q}$ and hence $\operatorname{deg}_{\mathbb{F}_{p^{2}}}(t):=\left[\mathbb{F}_{p^{2}}(t): \mathbb{F}_{p^{2}}\right] \leq 2(p+1)$. We need the following well-known result.

Theorem A.1 (Kummer's Theorem). Let $K$ be any field and $n \geq 1$ an integer and $a \in K^{\times}$. If $(n, \operatorname{char} K)=1$, and $\mu_{n}\left(K^{\mathrm{sep}}\right) \subseteq K$, and the element $a\left(\bmod \left(K^{\times}\right)^{n}\right)$ in $K^{\times} /\left(K^{\times}\right)^{n}$ has order $n$, then $\left[K\left(a^{1 / n}\right): K\right]=n$.

The authors are grateful to Ming-Lun Hsieh for providing the following proposition. 
Proposition A.2. There exist a conic $Q \in \mathscr{Q}$ and a point $t \in C \cap Q$ such that $\operatorname{deg}_{\mathbb{F}_{p^{2}}}(t)=$ $(p+1)$.

Proof. Choose a generator $u_{1}$ of $\mathbb{F}_{p^{2}}^{\times}$such that $u_{1}^{p}+u_{1}=-a \neq 0$. Put $u:=a^{-1} u_{1}$ and let $\alpha$ be a $p+1$-th root of $u$. As $a \in \mathbb{F}_{p}^{\times}$, we have $u^{p}+u=-1$. Since the element $u$ $\left(\bmod \left(\mathbb{F}_{p^{2}}^{\times}\right)^{p+1}\right)$ in $\mathbb{F}_{p^{2}}^{\times} /\left(\mathbb{F}_{p^{2}}^{\times}\right)^{p+1}=\mathbb{F}_{p^{2}}^{\times} /\left(\mathbb{F}_{p}^{\times}\right)$has order $p+1$, one has $\left[\mathbb{F}_{p^{2}}(\alpha): \mathbb{F}_{p^{2}}\right]=p+1$ by Kummer's Theorem. Let

$$
Q: X_{1} X_{2}=u X_{3}^{2} \quad \text { and } \quad t:=\left(\alpha: u \alpha^{-1}: 1\right)
$$

One sees $t \in C$ as $\alpha^{p+1}+\left(u \alpha^{-1}\right)^{p+1}+1=u+u^{p+1} \cdot u^{-1}+1=0$. So $t \in C \cap Q$ and $\operatorname{deg}_{\mathbb{F}_{p^{2}}}(t)=p+1$.

The following result, due to Akio Tamagawa, says that the upper bound $2(p+1)$ for $\operatorname{deg}_{\mathbb{F}_{p^{2}}}(t)$ in $C \cap \Delta$ can be realised.

Proposition A.3. There exist a conic $Q \in \mathscr{Q}$ and a point $t \in C \cap Q$ such that $\operatorname{deg}_{\mathbb{F}_{p^{2}}}(t)=$ $2(p+1)$.

Construction. We first consider the case $p=2$. Let $\zeta$ be a primitive fifth roof of unity in $\overline{\mathbb{F}}_{2}$. Since $(\mathbb{Z} / 5 \mathbb{Z})^{\times} \simeq\langle 2 \bmod 5\rangle$, we have $\mathbb{F}_{2}(\zeta)=\mathbb{F}_{2^{4}}$. One computes that $(1+\zeta)^{3}=$ $1+\zeta+\zeta^{2}+\zeta^{3} \neq 1$ and $(1+\zeta)^{5}=\zeta+\zeta^{4} \neq 1$. Therefore $1+\zeta$ generates the cyclic group $\mathbb{F}_{2^{4}}^{\times} \simeq C_{15}$. Choose $x, y, z \in \overline{\mathbb{F}}_{2}$ such that $x=1, y^{3}=\zeta$ and $z^{3}=1+\zeta$, and put $t:=(x: y: z)$; we have $1+\zeta+(1+\zeta)=0$. Since $\mathbb{F}_{2}(z)$ contains $\mathbb{F}_{2}(\zeta)=\mathbb{F}_{2^{4}}$, we have $\mathbb{F}_{2}(z)=\mathbb{F}_{2^{4}}(z)$. Since $\langle 1+\zeta\rangle=\mathbb{F}_{2^{4}}^{\times}$, by Kummer's Theorem, $\mathbb{F}_{2}(z)=\mathbb{F}_{2^{4}}(z)=\mathbb{F}_{2^{12}}$ and hence $\operatorname{deg}_{\mathbb{F}_{4}}(t)=6=2(p+1)$. Since $x, y \in \mathbb{F}_{2^{4}}$, there exist $a, b, c \in \mathbb{F}_{2^{2}}$ such that $a x^{2}+b x y+c y^{2}=0$. Let $Q \subseteq \mathbb{P}^{2}$ be the (degenerate) conic defined by the equation $a X_{1}^{2}+b X_{1} X_{2}+c X_{2}^{2}$. Then the point $t \in C \cap Q$ satisfies the desired property.

Assume now that $p>2$. We would like to find solutions $t=(x: y: z)$ with $x \in$ $\mathbb{F}_{p^{4(p+1)}}^{\times}, y \in \mathbb{F}_{p^{4}}^{\times} \backslash \mathbb{F}_{p^{2}}^{\times}$, and $z \in \mathbb{F}_{p^{2}}^{\times}$satisfying the desired properties.

Let

$$
f: \mathbb{F}_{p^{4}}^{\times} \rightarrow \mathbb{F}_{p^{4}}^{\times} /\left(\mathbb{F}_{p^{4}}^{\times}\right)^{2(p+1)}
$$

be the natural projection; one has $\mathbb{F}_{p^{4}}^{\times} /\left(\mathbb{F}_{p^{4}}^{\times}\right)^{2(p+1)} \simeq C_{2(p+1)}$ as $p \neq 2$. Consider the following three sets:

$$
\begin{aligned}
& Z:=\left\{z^{p+1}: z \in \mathbb{F}_{p^{2}}^{\times}\right\} \simeq \mathbb{F}_{p}^{\times} ; \\
& Y:=\left\{y^{p+1}: y \in \mathbb{F}_{p^{4}}^{\times}\right\} \backslash Z ; \\
& X:=\left\{\xi \in \mathbb{F}_{p^{4}}^{\times}: f(\xi) \text { generates the cyclic group } C_{2(p+1)}\right\} .
\end{aligned}
$$


The sets $Y$ and $Z$ are equipped with an $\mathbb{F}_{p}^{\times}$-action and we have

$$
|Z|=p-1, \quad|Y|=p^{2}(p-1), \quad|X|=\left(p^{4}-1\right) \cdot \frac{\varphi(2(p+1))}{2(p+1)} .
$$

Let $g$ be the composition

$$
g: \mathbb{F}_{p^{4}}^{\times} \stackrel{N}{\longrightarrow} \mathbb{F}_{p^{2}}^{\times} \stackrel{\text { proj. }}{\longrightarrow} \mathbb{F}_{p^{2}}^{\times} /\left(\mathbb{F}_{p}^{\times}\right)^{2} \simeq C_{2(p+1)},
$$

where $N(\alpha)=\alpha^{p^{2}+1}$ is the norm map. The map $f$ can be identified with $g$ by a suitable choice of the generators. Since the image $g\left(\mathbb{F}_{p}^{\times}\right)$is trivial, the image $f\left(\mathbb{F}_{p}^{\times}\right)$is also trivial. Thus, $X$ is also equipped with an $\mathbb{F}_{p}^{\times}$-action and hence $-X=X$.

We would like to find

$$
\eta+\zeta=\xi
$$

for some $\eta \in Y, \zeta \in Z$ and $\xi \in-X=X$.

Note that $X, Y$ and $Z$ are mutually disjoint: that $Y \cap Z=\emptyset$ follows by definition, and $X \cap Z=\emptyset$ follows from the fact that $\mathbb{F}_{p}^{\times} \subseteq \operatorname{ker}(f)$. Since $f\left(\left(\mathbb{F}_{p^{4}}^{\times}\right)^{p+1}\right)$ is the 2-torsion subgroup of $\mathbb{F}_{p^{4}}^{\times} /\left(\mathbb{F}_{p^{4}}^{\times}\right)^{2(p+1)} \simeq C_{2(p+1)}$ and $f(Y) \subseteq f\left(\left(\mathbb{F}_{p^{4}}^{\times}\right)^{p+1}\right)$, the image $f(Y)$ contains no generator of $C_{2(p+1)}$. Therefore, we also have $Y \cap X=\emptyset$.

We are working on the space $\mathbb{P}:=\mathbb{F}_{p^{4}}^{\times} / \mathbb{F}_{p}^{\times} \simeq \mathbb{P}^{3}\left(\mathbb{F}_{p}\right)$. The images of $X, Y$ and $Z$ in $\mathbb{P}$ are written as $\bar{X}, \bar{Y}$ and $\bar{Z}$, respectively. So $\bar{Z}=\{\bar{\zeta}\}$ and

$$
|\bar{Z}|=1, \quad|\bar{Y}|=p^{2}, \quad|\bar{X}|=\left(p^{2}+1\right) \cdot \frac{\varphi(2(p+1))}{2} .
$$

For each point $\bar{\eta} \in \bar{Y}(\bar{\eta} \neq \bar{\zeta})$, denote by $L_{\bar{\eta}} \subseteq \mathbb{P}$ the line joining the points $\bar{\eta}$ and $\bar{\zeta}$. To solve (74), it suffices to prove that

$$
\left(\bigcup_{\bar{\eta} \in \bar{Y}} L_{\bar{\eta}}\right) \cap \bar{X} \neq \emptyset
$$

This is because if $\bar{\xi} \in L_{\bar{\eta}} \cap \bar{X}$ for some $\bar{\eta} \in \bar{Y}$, then we have $a \eta+b \zeta=c \xi$ with $a, b, c \in \mathbb{F}_{p}^{\times}$ and hence $\eta^{\prime}+\zeta^{\prime}=\xi^{\prime}$ with $\eta^{\prime} \in Y, \zeta^{\prime} \in Z$ and $\xi^{\prime} \in X$.

Lemma A.4. For any two distinct points $\bar{\eta}_{1}$ and $\bar{\eta}_{2}$ of $\bar{Y}$, one has $L_{\bar{\eta}_{1}} \cap L_{\bar{\eta}_{2}}=\{\bar{\zeta}\}$.

Proof. Suppose that $L_{\bar{\eta}_{1}} \cap L_{\bar{\eta}_{2}} \supsetneq\{\bar{\zeta}\}$. Then $L_{\bar{\eta}_{1}}=L_{\bar{\eta}_{2}}$ and $\bar{\eta}_{2} \in L_{\bar{\eta}_{1}}$. Therefore, $-\eta_{2}=$ $a \eta_{1}+b \zeta$ for $a, b \in \mathbb{F}_{p}^{\times}$and hence we have

$$
\eta_{2}+\eta_{1}^{\prime}+\zeta^{\prime}=0
$$


for some $\eta_{1}^{\prime} \in Y$ and $\zeta^{\prime} \in Z$. Now write

$$
\eta_{2}=\left(y_{2}\right)^{p+1}, \quad \eta_{1}^{\prime}=\left(y_{1}^{\prime}\right)^{p+1}, \quad \zeta^{\prime}=\left(z^{\prime}\right)^{p+1}
$$

with $y_{2}, y_{1}^{\prime} \in \mathbb{F}_{p^{4}}^{\times} \backslash \mathbb{F}_{p^{2}}^{\times}$and $z^{\prime} \in \mathbb{F}_{p^{2}}^{\times}$. That is, we get a point $\left(y_{2}: y_{1}^{\prime}: z^{\prime}\right) \in C\left(\mathbb{F}_{p^{4}}\right)$. Since $C\left(\mathbb{F}_{p^{4}}\right)=C\left(\mathbb{F}_{p^{2}}\right)$ by Lemma 3.8, we have $y_{2}, y_{1}^{\prime} \in \mathbb{F}_{p^{2}}$, contradiction.

By Lemma A.4,

$$
\bigcup_{\bar{\eta} \in \bar{Y}} L_{\bar{\eta}}=\{\bar{\zeta}\} \amalg \coprod_{\bar{\eta} \in \bar{Y}} L_{\bar{\eta}}-\{\bar{\zeta}\},
$$

and hence

$$
\left|\bigcup_{\bar{\eta} \in \bar{Y}} L_{\bar{\eta}}\right|=1+|\bar{Y}| \cdot p=p^{3}+1, \quad \text { and } \quad\left|\mathbb{P}-\bigcup_{\bar{\eta} \in \bar{Y}} L_{\bar{\eta}}\right|=p^{2}+p
$$

To show (74), we check the inequality

$$
|\bar{X}|=\left(p^{2}+1\right) \cdot \frac{\varphi(2(p+1))}{2}>p^{2}+p
$$

for all $p \neq 2$. If $p=3$, then $|\bar{X}|=20>12$ holds. For $p \geq 5$, by the inequality $\varphi(n) \geq \sqrt{n / 2}$, it suffices to show

$$
\left(p^{2}+1\right) \cdot \frac{\sqrt{p+1}}{2}>p^{2}+p
$$

This follows from

$$
\left(p^{2}+1\right)^{2}(p+1)-4\left(p^{2}+p\right)^{2}=(p+1)\left(p^{4}-4 p^{3}-2 p^{2}+1\right)>0
$$

for $p \geq 5$. Therefore, the inequality (76) holds and we have found $\eta, \zeta, \xi$ as in (74).

Now write

$$
\zeta=z^{p+1} \quad\left(\text { for } z \in \mathbb{F}_{p^{2}}^{\times}\right), \quad \eta=y^{p+1} \quad\left(\text { for } y \in \mathbb{F}_{p^{4}}^{\times} \backslash \mathbb{F}_{p^{2}}^{\times}\right)
$$

Choose an element $x \in \overline{\mathbb{F}}_{p}$ such that $x^{p+1}=-\xi \in \mathbb{F}_{p^{4}}^{\times}$. Since the element $\xi$ $\left(\bmod \left(\mathbb{F}_{p^{4}}^{\times}\right)^{p+1}\right)$ is a generator in $\mathbb{F}_{p^{4}}^{\times} /\left(\mathbb{F}_{p^{4}}^{\times}\right)^{p+1}$, by Kummer's Theorem we have

$$
\left[\mathbb{F}_{p^{4}}(x): \mathbb{F}_{p^{4}}\right]=p+1 \text {. }
$$

We claim that $\xi \notin \mathbb{F}_{p^{2}}^{\times}$. Suppose for contradiction that $\xi \in \mathbb{F}_{p^{2}}^{\times}$. Then

$$
f(\xi)=g(\xi) \in g\left(\mathbb{F}_{p^{2}}^{\times}\right)=\left(\mathbb{F}_{p^{2}}^{\times}\right)^{2} /\left(\mathbb{F}_{p}^{\times}\right)^{2} \subsetneq \mathbb{F}_{p^{2}}^{\times} /\left(\mathbb{F}_{p}^{\times}\right)^{2} \simeq C_{2(p+1)} .
$$


Therefore, $f(\xi)$ cannot be a generator of $C_{2(p+1)}$, contradiction. So since $\xi \in \mathbb{F}_{p^{4}}^{\times} \backslash \mathbb{F}_{p^{2}}^{\times}$, we have $\mathbb{F}_{p^{2}}(x) \supset \mathbb{F}_{p^{2}}(\xi)=\mathbb{F}_{p^{4}}$. This shows that

$$
\mathbb{F}_{p^{2}}(x)=\mathbb{F}_{p^{4}}(x), \quad \text { and } \quad\left[\mathbb{F}_{p^{2}}(x): \mathbb{F}_{p^{2}}\right]=2(p+1)
$$

by (77). Put $t:=(x: y: z)=(x / z: y / z: 1) \in C\left(\overline{\mathbb{F}}_{p}\right)$. Then we get

$$
\left[\mathbb{F}_{p^{2}}(t): \mathbb{F}_{p^{2}}\right]=2(p+1)
$$

Since $y / z \in \mathbb{F}_{p^{4}}^{\times} \backslash \mathbb{F}_{p^{2}}^{\times}$, there exist $b, c \in \mathbb{F}_{p^{2}}$ such that

$$
\left(\frac{y}{z}\right)^{2}+b\left(\frac{y}{z}\right)+c=0, \quad \text { or } \quad y^{2}+b y z+c z^{2}=0
$$

Let $Q \in \mathscr{Q}$ be the (degenerate) conic defined by the equation $X_{2}^{2}+b X_{2} X_{3}+c X_{3}^{2}=0$. Then $t \in C \cap Q$ and $\operatorname{deg}_{\mathbb{F}_{p^{2}}}(t)=2(p+1)$. This completes the construction.

\section{A.2. Estimate of $|C \cap \Delta|$}

In this subsection, points in $C$ will mean geometric points and $C \cap \Delta$ will mean the set-theoretic intersection. Define

$$
\mathscr{Z}:=\{(t, Q) \in C \times \mathscr{Q}: t \in \mathscr{Q}\}
$$

and consider the following natural maps:

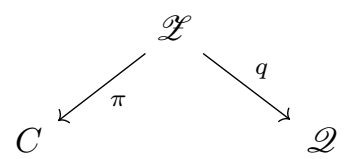

The degree of the map $q$ is $2(p+1)$. For each $Q \in \mathscr{Q}$, the fibre over $Q$ has size

$$
2(p+1)-\varepsilon_{Q}
$$

where $\varepsilon_{Q}=\sum_{r \geq 2} \varepsilon_{Q, r}$ with

$$
\varepsilon_{Q, r}=\#\left\{t \in C \cap Q: \operatorname{mult}_{C \cap \Delta}(t)=r\right\} \cdot(r-1) .
$$

Thus, $|\mathscr{Z}|=2(p+1)\left(p^{10}+p^{8}+p^{6}+p^{4}+p^{2}+1\right)-\varepsilon$, where

$$
\varepsilon:=\sum_{Q \in \mathscr{Q}} \varepsilon_{Q}
$$

is the error term coming from intersection multiplicities. 
Proposition A.5. We have $|C \cap \Delta|=p^{11}+o\left(p^{11}\right)-\varepsilon$ as $p \rightarrow \infty$, where $\varepsilon$ is defined in (79).

Remark A.6. We expect that $\varepsilon=o\left(p^{11}\right)$. Then we would have $|C \cap \Delta|=p^{11}+o\left(p^{11}\right)$ as $p \rightarrow \infty$.

Proof. For any integer $i \geq 1$, define

$$
C_{i}:=\left\{t \in C\left(\overline{\mathbb{F}}_{p}\right): \operatorname{deg}_{\mathbb{F}_{p^{2}}}(t)=i\right\}
$$

By Lemma 3.8, we have

$$
\begin{gathered}
\left|C_{1}\right|=\left|C\left(\mathbb{F}_{p^{2}}\right)\right|=p^{3}+1, \quad\left|C_{3}\right|=\left|C^{0}\left(\mathbb{F}_{p^{6}}\right)\right|=p^{6}+p^{5}-p^{4}-p^{3}, \\
\left|C_{4}\right|=\left|C^{0}\left(\mathbb{F}_{p^{8}}\right)\right|=p^{8}-p^{6}+p^{5}-p^{3}, \quad\left|C_{5}\right|=\left|C^{0}\left(\mathbb{F}_{p^{10}}\right)\right|=p^{10}+p^{7}-p^{6}-p^{3} .
\end{gathered}
$$

Let $\mathbb{F}_{p^{2}}\left[X_{1}, X_{2}, X_{3}\right]_{2} \subseteq \mathbb{F}_{p^{2}}\left[X_{1}, X_{2}, X_{3}\right]$ denote the subspace of homogeneous polynomials of degree two. For each point $t=\left(t_{1}: t_{2}: t_{3}\right) \in C$, the fibre $\pi^{-1}(t)$ is the set $\left(W_{t}-\{0\}\right) / \mathbb{F}_{p^{2}}^{\times}$, where

$$
W_{t}:=\left\{F \in \mathbb{F}_{p^{2}}\left[X_{1}, X_{2}, X_{3}\right]_{2}: F(t)=0\right\}
$$

They fit into the following exact sequence

$$
0 \longrightarrow W_{t} \longrightarrow \mathbb{F}_{p^{2}}\left[X_{1}, X_{2}, X_{3}\right]_{2} \stackrel{\mathrm{ev}_{t}}{\longrightarrow} \mathbb{F}_{p^{2}}\left\langle t_{1}^{2}, t_{2}^{2}, t_{3}^{2}, t_{1} t_{2}, t_{1} t_{3}, t_{2} t_{3}\right\rangle \longrightarrow 0
$$

It follows that $\operatorname{dim}\left(W_{t}\right)=6-d(t)$ and $\pi^{-1}(t) \simeq \mathbb{P}^{5-d(t)}\left(\mathbb{F}_{p^{2}}\right)$, where we redefine $d(t)$ as the dimension of $\mathbb{F}_{p^{2}}\left\langle t_{1}^{2}, t_{2}^{2}, t_{3}^{2}, t_{1} t_{2}, t_{1} t_{3}, t_{2} t_{3}\right\rangle$ - even for $p=2$. Therefore, the numbers of fibres over $C_{i}$ for $i=1,3,4,5$ are

$$
\left(p^{8}+p^{6}+p^{4}+p^{2}+1\right), \quad\left(p^{4}+p^{2}+1\right), \quad\left(p^{2}+1\right), \quad 1,
$$

respectively. Then the number of points in $\mathscr{Z}$ over the union of $C_{i}$ for $i=1,3,4,5$ is given by

$$
\begin{aligned}
A:= & \left(p^{3}+1\right)\left(p^{8}+p^{6}+p^{4}+p^{2}+1\right)+\left(p^{6}+p^{5}-p^{4}-p^{3}\right)\left(p^{4}+p^{2}+1\right) \\
& +\left(p^{8}-p^{6}+p^{5}-p^{3}\right)\left(p^{2}+1\right)+\left(p^{10}+p^{7}-p^{6}-p^{3}\right) \\
= & p^{11}+3 p^{10}+2 p^{9}+p^{8}+3 p^{7}-p^{6}+p^{5}-2 p^{3}+p^{2}+1 .
\end{aligned}
$$

Thus,

$$
\begin{aligned}
B & :=\#\left\{(t, Q) \in \mathscr{Z}: \operatorname{deg}_{\mathbb{F}_{p^{2}}}(t)>5\right\}=|\mathscr{Z}|-A \\
& =p^{11}-p^{10}+p^{8}-p^{7}+3 p^{6}+p^{5}+2 p^{4}+4 p^{3}+p^{2}+2 p+1-\varepsilon
\end{aligned}
$$




\section{Finally,}

$$
\begin{aligned}
|C \cap \Delta| & =|\operatorname{Im}(\pi)|=\left|C_{1}\right|+\left|C_{3}\right|+\left|C_{4}\right|+\left|C_{5}\right|+B \\
& =p^{11}+2 p^{8}+2 p^{6}+3 p^{5}+p^{4}+2 p^{3}+p^{2}+2 p+2-\varepsilon .
\end{aligned}
$$

\section{References}

[1] M. Deuring, Die Typen der Multiplikatorenringe elliptischer Funktionenkörper, Abh. Math. Sem. Hansischen Univ. 14 (1941) 197-272.

[2] S.J. Edixhoven, B.J.J. Moonen, F. Oort, Open problems in algebraic geometry, Bull. Sci. Math. 125 (1) (2001) 1-22.

[3] T. Ekedahl, On supersingular curves and abelian varieties, Math. Scand. 60 (2) (1987) 151-178.

[4] S. Harashita, Ekedahl-Oort strata contained in the supersingular locus and Deligne-Lusztig varieties, J. Algebraic Geom. 19 (3) (2010) 419-438.

[5] K. Hashimoto, Class numbers of positive definite ternary quaternion Hermitian forms, Proc. Jpn. Acad., Ser. A, Math. Sci. 59 (10) (1983) 490-493.

[6] K. Hashimoto, T. Ibukiyama, On class numbers of positive definite binary quaternion Hermitian forms, J. Fac. Sci., Univ. Tokyo, Sect. 1A, Math. 27 (3) (1980) 549-601.

[7] T. Ibukiyama, On automorphism groups of positive definite binary quaternion Hermitian lattices and new mass formula, in: Automorphic Forms and Geometry of Arithmetic Varieties, in: Adv. Stud. Pure Math., vol. 15, Academic Press, Boston, MA, 1989, pp. 301-349.

[8] T. Ibukiyama, Principal polarizations of supersingular abelian surfaces, J. Math. Soc. Jpn. 72 (4) (2020) 1161-1180.

[9] T. Ibukiyama, T. Katsura, F. Oort, Supersingular curves of genus two and class numbers, Compos. Math. 57 (2) (1986) 127-152.

[10] J. Igusa, Class number of a definite quaternion with prime discriminant, Proc. Natl. Acad. Sci. USA 44 (1958) 312-314.

[11] T. Katsura, F. Oort, Families of supersingular abelian surfaces, Compos. Math. 62 (2) (1987) $107-167$.

[12] T. Katsura, F. Oort, Supersingular abelian varieties of dimension two or three and class numbers, in: Algebraic Geometry, Sendai, 1985, in: Adv. Stud. Pure Math., vol. 10, North-Holland, Amsterdam, 1987 , pp. 253-281.

[13] K.-Z. Li, F. Oort, Moduli of Supersingular Abelian Varieties, Lecture Notes in Mathematics, vol. 1680, Springer-Verlag, Berlin, 1998.

[14] D. Mumford, Abelian Varieties, Tata Institute of Fundamental Research Studies in Mathematics, vol. 5, 2008, Published for the Tata Institute of Fundamental Research, Bombay, with appendices by C.P. Ramanujam and Yuri Manin, corrected reprint of the second (1974) edition.

[15] F. Oort, Which abelian surfaces are products of elliptic curves?, Math. Ann. 214 (1975) 35-47.

[16] F. Oort, Hyperelliptic supersingular curves, in: Arithmetic Algebraic Geometry, Texel, 1989, in: Progr. Math., vol. 89, Birkhäuser Boston, Boston, MA, 1991, pp. 247-284.

[17] F. Oort, Foliations in moduli spaces of abelian varieties, J. Am. Math. Soc. 17 (2) (2004) $267-296$.

[18] M. Rapoport, Th. Zink, Period Spaces for p-Divisible Groups, Ann. Math. Studies, vol. 141, Princeton Univ. Press, 1996.

[19] M.-F. Vignéras, Arithmétique des algèbres de quaternions, Lecture Notes in Mathematics, vol. 800, Springer, Berlin, 1980.

[20] J.W. Xue, C.-F. Yu, On counting certain abelian varieties over finite fields, Acta Math. Sin. Engl. Ser. 37 (2021) 205-228.

[21] C.-F. Yu, On the supersingular locus in Hilbert-Blumenthal 4-folds, J. Algebraic Geom. 12 (4) (2003) 653-698.

[22] C.-F. Yu, On the mass formula of supersingular abelian varieties with real multiplications, J. Aust. Math. Soc. 78 (3) (2005) 373-392.

[23] C.-F. Yu, The supersingular loci and mass formulas on Siegel modular varieties, Doc. Math. 11 (2006) 449-468.

[24] C.-F. Yu, On finiteness of endomorphism rings of abelian varieties, Math. Res. Lett. 17 (2) (2010) $357-370$. 
[25] C.-F. Yu, Simple mass formulas on Shimura varieties of PEL-type, Forum Math. 22 (3) (2010) $565-582$.

[26] C.-F. Yu, On fields of definition of components of the Siegel supersingular locus, Proc. Am. Math. Soc. 145 (12) (2017) 5053-5058.

[27] C.-F. Yu, J.-D. Yu, Mass formula for supersingular abelian surfaces, J. Algebra 322 (10) (2009) 3733-3743. 